\title{
Consistency of the standard model effective field theory
}

\author{
Grant N. Remmen and Nicholas L. Rodd \\ Center for Theoretical Physics and Department of Physics, \\ University of California, Berkeley, CA 94720, U.S.A. \\ Lawrence Berkeley National Laboratory, \\ Berkeley, CA 94720, U.S.A. \\ E-mail: grant.remmen@berkeley.edu, nrodd@berkeley.edu
}

ABStRaCT: We derive bounds on couplings in the standard model effective field theory (SMEFT) as a consequence of causality and the analytic structure of scattering amplitudes. In the SMEFT, there are 64 independent operators at mass dimension eight that are quartic in bosons (either Higgs or gauge fields) and that contain four derivatives and/or field strengths, including both $\mathrm{CP}$-conserving and $\mathrm{CP}$-violating operators. Using analytic dispersion relation arguments for two-to-two bosonic scattering amplitudes, we derive 27 independent bounds on the sign or magnitude of the couplings. We show that these bounds also follow as a consequence of causality of signal propagation in nonvacuum SM backgrounds. These bounds come in two qualitative forms: i) positivity of (various linear combinations of) couplings of CP-even operators and ii) upper bounds on the magnitude of CP-odd operators in terms of (products of) CP-even couplings. We exhibit various classes of example completions, which all satisfy our EFT bounds. These bounds have consequences for current and future particle physics experiments, as part of the observable parameter space is inconsistent with causality and analyticity. To demonstrate the impact of our bounds, we consider applications both to SMEFT constraints derived at colliders and to limits on the neutron electric dipole moment, highlighting the connection between such searches suggested by infrared consistency.

Keywords: Beyond Standard Model, Effective Field Theories, CP violation

ArXiv EPrint: 1908.09845 


\section{Contents}

1 Introduction 1

2 Infrared consistency 4

3 Operator basis $\quad 8$

3.1 Four-derivative dimension-eight bosonic operators 8

$\begin{array}{lll}3.2 & \text { Lower-dimension operators } & 13\end{array}$

4 Bounds $\quad \mathbf{1 5}$

$\begin{array}{lll}4.1 & \text { Single field strength quartics } & 15\end{array}$

$\begin{array}{lll}4.1 .1 & \mathrm{SU}(3) & 18\end{array}$

$\begin{array}{lll}4.1 .2 & \mathrm{SU}(2) & 18\end{array}$

$\begin{array}{lll}4.1 .3 & \mathrm{U}(1) & 18\end{array}$

$\begin{array}{lll}4.2 & \text { Field strength cross-quartics } & 19\end{array}$

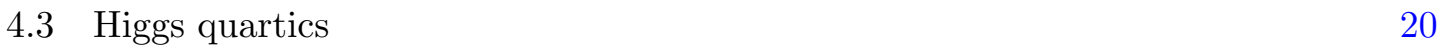

4.4 Higgs/field strength cross-quartics 22

5 UV completions $\quad \mathbf{2 3}$

5.1 One-loop completions of gauge field operators 23

$\begin{array}{lll}5.2 & \text { Born-Infeld } & 26\end{array}$

5.3 CP-violating terms and completing the square 27

5.4 Tree-level completions of $(D H)^{4}$ operators 29

6 Phenomenological consequences $\quad 31$

6.1 Anomalous quartic gauge-boson couplings 32

6.2 Neutron EDM 38

$\begin{array}{lll}7 & \text { Conclusions } & 40\end{array}$

A Group theory definitions $\quad 41$

\section{Introduction}

We are currently entering a unique era in particle physics. With the discovery of the Higgs boson $[1,2]$, the Large Hadron Collider (LHC) has completed the detection of all of the fundamental constituents of the standard model (SM). The absence as of yet of detections of supersymmetric (SUSY) partner states [3] — and the possible dawn of a post-naturalness era [4-6] - means that the road ahead for model building is both wide open and unclear. Without requiring a large number of SUSY states at the weak scale, possible beyond-SM 
(BSM) theories run the gamut from a rich structure of fields around the corner, near a $\mathrm{TeV}$, to a desert up to a very high scale (see, e.g., refs. [7,8] for illustrative examples of these alternatives). At the same time, a few clear signals of BSM physics are presently indicated, namely, dark matter, neutrino masses, and baryogenesis.

Faced with this situation, it is conceivable that the first tangible discoveries of BSM physics will come not in the form of new on-shell particle states, but instead through the detection of deviations from the SM through precision experiments, including the high luminosity/precision era at the LHC [9-12], Higgs factories in the form of future $e^{+} e^{-}$ colliders (see ref. [13] and refs. therein), measurements of the neutron and electron electric dipole moments (EDMs) [14-19], and the muon $g-2$ [20, 21], among many others. Such deviations from the SM, induced by new high-scale physics, can be encoded as operators of higher mass dimension in an effective field theory (EFT) of the SM [22-26]. Using current measurements from the LHC [27-32], bounds have begun to be placed on the Wilson coefficients of some of the operators in the SMEFT; for a single example see ref. [33].

In constructing an EFT, the Wilsonian approach is to write down all of the operators allowed, constrained only by Lorentz and gauge invariance (along with any other exact symmetries of the EFT), with arbitrary coefficients. However, not all infrared (IR) Lagrangians can be generated by healthy ultraviolet (UV) completions. As shown in ref. [34], ${ }^{1}$ causality of signal propagation, as well as quantum mechanical unitarity and the analytic properties of scattering amplitudes, constrains the Wilson coefficients in the EFT; see also refs. [38, 39]. This requirement of IR consistency has led to a program of bounding couplings in various EFTs of interest, including corrections to general relativity [40-43], nonlinear electrodynamics [34], massive gravity [44-47] and higher-spin states [48-54], certain scalar theories [55-59] including the proof of the four-dimensional $a$-theorem [60], theories of fermion compositeness [61], electroweak chiral Lagrangian [62, 63], chiral perturbation theory [35-37], the electroweak EFT [64-66], and Einstein-Maxwell theory with applications to the Weak Gravity Conjecture [67-71]. The IR consistency program, taking a bottom-up approach to constraining EFTs, has evolved in tandem with the swampland program [72-74], which constrains EFTs compatible with quantum gravity via a top-down perspective, examining the space of string theoretic UV completions.

Despite this progress, IR consistency bounds from analyticity and causality have not been systematically applied to the full SMEFT itself. The present is an especially compelling time to do so, given that bounding and measuring SMEFT coefficients will be a focus of current and future precision particle physics experiments. The consistency constraints can be applied to the results of such experiments in at least three ways. First, constraining the space of possible EFT coefficients leads to enhanced statistical power for experiments sensitive to these operators, since one can incorporate IR consistency bounds into the prior probability distribution. A schematic depiction of this interplay between the bounds derived in this work and experimental constraint is shown in figure 1. In total we will derive 27 independent bounds; if each were as simple as requiring a certain operator coefficient to be positive, then this would represent a factor of $2^{27} \sim 10^{8}$ reduction in the

\footnotetext{
${ }^{1}$ See also refs. [35-37] for earlier applications of such arguments to chiral perturbation theory.
} 


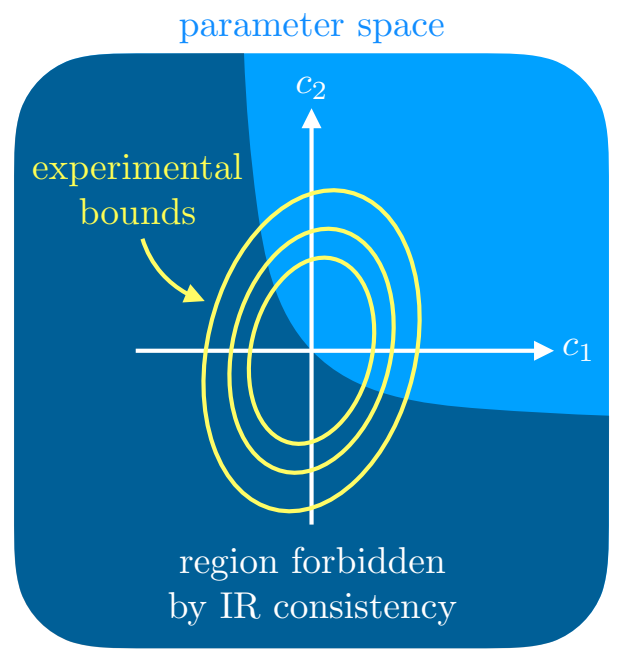

Figure 1. Schematic depiction of bounds derived in this work. For the example of an observable sensitive to two SMEFT operators, $\mathcal{O}_{1}$ and $\mathcal{O}_{2}$, data can weigh directly on the allowed parameter space for these operators, as shown by the yellow contours in the figure. However, much of the parameter space is inconsistent with fundamental underlying properties of quantum field theory, and any theory with couplings in the forbidden region would depart significantly from standard assumptions about the form of UV physics (causality, unitarity, etc.). Consequently, our bounds can be viewed as placing strong theoretical priors on the parameter space of the SMEFT.

parameter space. This is just a rough estimate, however, because the bounds will often appear as relations between the various operators. For example, we will see a number of examples where the size of CP-odd operators is bounded from above by certain CP-even contributions. This implies a second possible use for our bounds: linking naively disparate experimental measurements. We will explore an example of this in the form of connecting collider searches to measurements of the neutron EDM. A third application of our results is that a definitive discovery of new physics, appearing in the form of nonzero SMEFT coefficients, would allow our bounds to be directly compared with data (provided that the sign of the operator coefficients can be determined). Testing whether the resulting coefficients satisfy our bounds thereby allows for fundamental properties of the UV theory such as causality, analyticity/locality, unitarity, and Lorentz invariance - to be tested to very high energies through experimental signatures at accessible scales.

Although we will not attempt an exhaustive exploration of the experimental consequences of our results, one example we will consider in more detail is the search for BSM contributions to electroweak boson four-point vertices, such as $W W \gamma Z$. Such corrections are traditionally referred to as anomalous quartic gauge-boson couplings (aQGCs) and include all possible four-gauge-boson vertices in the electroweak sector of the SM (i.e., excluding gluons). Both CMS and ATLAS are actively searching for processes sensitive to the quartic gauge-boson vertices and aQGCs; see ref. [75] for a review. The dimension-eight SMEFT operators that induce aQGCs, first written down in ref. [76], are a subset of the operators we will consider here. As such, the bounds we derive in this work forbid cou- 
plings in certain regions of parameter space; while experimentally accessible, these regions are impossible to generate via any conventional UV completion. We note that positivity constraints on these operators were also recently considered in refs. $[65,66]$, which we will discuss in section 6.1. In much of the experimental and phenomenological literature on aQGCs, a one-at-a-time assumption is employed, where constraints are derived by allowing only one higher-dimension operator to be nonzero; this assumption strongly limits the applicability and genericity of such bounds to arbitrary EFTs. We will show that even without using this simplifying assumption, we are able to derive bounds on aQGCs that are both simple and general.

In this paper, we will consider bosonic higher-dimension operators in the SMEFT. In order to bound operators using analyticity of scattering amplitudes or causality of particle propagation, we will specialize to gauge-invariant operators that contain terms quartic in the fields [34]. By derivative counting, we require operators with a number of derivatives (possibly inside field strengths) divisible by four in order to obtain positivity bounds from forward amplitudes or causal dispersion relations. Hence, we will be placing positivity bounds on dimension-eight operators, which have Wilson coefficients $\sim 1 / M^{4}$ for $M$ the cutoff scale at which new physics enters.

A priori, one could also consider dimension-six operators, which are formally dominant, scaling as $1 / M^{2}$ (for progress in this direction, see e.g. refs. [77, 78]). Indeed, generically two insertions of a dimension-six operator would compete with dimension-eight terms. In such cases, the squared contribution of the dimension-six coupling would obstruct our ability to place positivity constraints directly on dimension-eight operators in the EFT. However, we will see in section 3.2 that, in the SMEFT, we can choose our scattering states to exclude the dimension-six contribution from interference with the operators of interest, leaving valid positivity bounds purely on the coefficients of dimension-eight operators.

This paper is structured as follows. In section 2 we briefly review the IR arguments resulting in positivity bounds on EFT coefficients from analyticity and causality. We give the basis of all dimension-eight four-boson operators in the SMEFT in section 3. In section 4 we derive our positivity bounds on bosonic operator coefficients in the SMEFT. As a check of our bounds, we consider broad classes of example UV completions of our operators in section 5. We explore a subset of the phenomenological implications in section 6 and conclude in section 7 .

\section{Infrared consistency}

In the interest of being self-contained, let us first briefly review how the analytic structure of scattering amplitudes and causality of signal propagation can be used to bound coefficients of operators in EFTs. For details, see ref. [34], as well as further discussion, extensions, and applications in refs. [38-60, 65-71]. Throughout this section, in order to keep the discussion grounded, we will periodically return to an application of the bounds to a particularly simple example: the theory of a single massless scalar field $\phi$, invariant under a shift symmetry $\phi \rightarrow \phi+$ constant. The Lagrangian for this theory, including the leading-order 
dimension-eight interaction, is given by

$$
\mathcal{L}=-\frac{1}{2}(\partial \phi)^{2}+\frac{c}{M^{4}}(\partial \phi)^{4}
$$

As we will review, consistency requires $c>0$. Throughout this work, we use the mostly-plus metric signature convention, $g_{\mu \nu}=\operatorname{diag}(-1,+1,+1,+1)$. For our key results, however, we will always identify if there is any dependence upon convention.

In order to establish the analyticity bound, consider the scattering of massless particles $12 \rightarrow 34$, with the incoming state $\left|\psi_{\text {in }}\right\rangle$ describing particles 1 and 2 . The two-to-two amplitude $\mathcal{M}(s, t)$ can be written as a function of the Mandelstam variables $s$ and $t,^{2}$ as well as (for a general theory) polarizations or other quantum numbers. The forward amplitude, which we will write as $\mathcal{A}(s)$, is obtained by taking $t \rightarrow 0$, as well as ensuring that the outgoing state $\left|\psi_{\text {out }}\right\rangle$ is identical to the incoming one (e.g., in polarizations, flavor, species, etc.). For our scalar field example, the two-to-two scattering amplitude is

$$
\mathcal{M}(s, t)=\frac{2 c}{M^{4}}\left(s^{2}+t^{2}+u^{2}\right)
$$

and so in this case, the forward amplitude is simply $\mathcal{A}(s)=4 c s^{2} / M^{4}$.

Returning to the general case, let us promote the squared center-of-mass energy $s$ to a complex quantity. The analytic structure of scattering amplitudes as functions of complex momenta has been well studied [79-83]; in particular, $\mathcal{A}(s)$ is analytic everywhere in the plane except near the real $s$ axis, where simple poles correspond to on-shell tree-level exchange of massive intermediate states, and branch cuts along the axis are associated with logarithmic terms arising from loops, i.e., multiparticle on-shell production. We can exploit this general structure as follows. Let us expand $\mathcal{A}(s)$ in an EFT as $\sum_{n=0}^{\infty} \lambda_{n} s^{n}$ at small $s$. Using this expansion and defining a small circular contour $\mathcal{C}$ around the origin, we can isolate the coefficient of $s^{2}$ in the forward amplitude using the following contour integral:

$$
\lambda_{2}=\frac{1}{2 \pi i} \oint_{\mathcal{C}} \frac{\mathrm{d} s}{s^{3}} \mathcal{A}(s) .
$$

We will now establish that $\lambda_{2}>0 .{ }^{3}$ To do so, we exploit the analyticity of $\mathcal{A}(s)$ to deform $\mathcal{C}$ to a new contour $\mathcal{C}^{\prime}$, which stretches out to infinity wherever it is not obstructed by the discontinuities in $\mathcal{A}(s)$, as shown in figure 2. For simplicity, we will treat the discontinuity as beginning at $\pm s_{\mathrm{d}}$. This choice is well motivated as it appears in simple UV completions. For example, a tree-level completion involving the exchange of a state with mass $m$ will generate two poles on the real $s$ axis, fixing $s_{\mathrm{d}}=m^{2}$. Further, if the theory is instead UV completed at one loop using a single particle of the same mass, then the associated branch cuts lead to $s_{\mathrm{d}}=4 \mathrm{~m}^{2}$. Nevertheless, in general the theory will contain poles as well as branch cuts, the exact locations of which are determined by the

\footnotetext{
${ }^{2}$ We use the all-incoming convention for momenta and define the Mandelstam variables as $s=-\left(p_{1}+p_{2}\right)^{2}$, $t=-\left(p_{1}+p_{3}\right)^{2}$, and $u=-\left(p_{1}+p_{4}\right)^{2}$, where $s+t+u=0$ for massless states.

${ }^{3}$ For simplicity of notation, throughout we will write all bounds on operator coefficients as strict inequalities, though this could be relaxed (i.e., $\geq$ ) if we are working at fixed (e.g., tree) order, cf. refs. [34, 55, 58].
} 


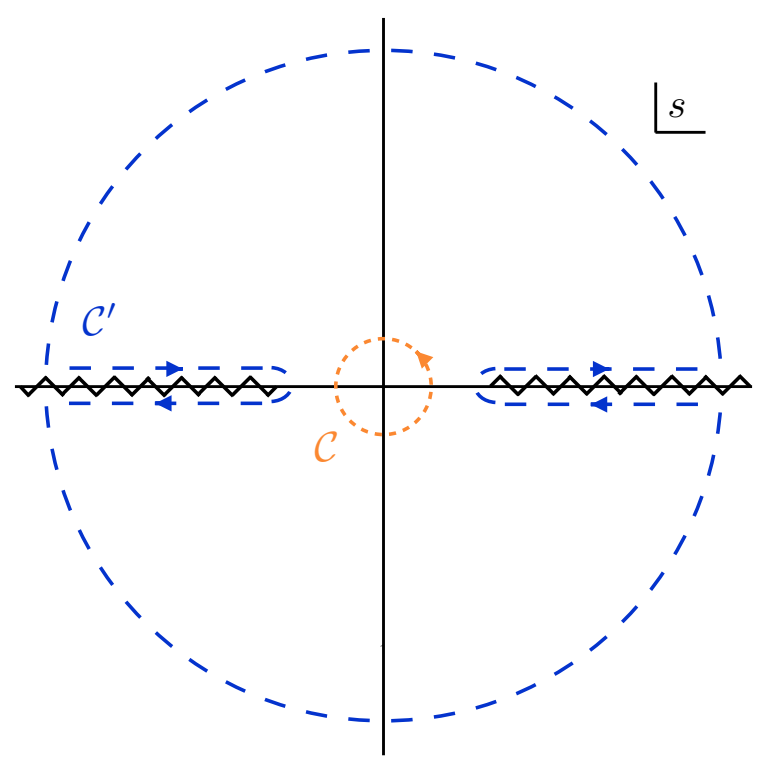

Figure 2. A schematic depiction of the analytic structure of the amplitude in the complex $s$ plane, as well as the two contours we will use to establish our positivity bounds. The zigzag lines denote the discontinuity in the amplitude, which can include poles and branch cuts.

UV properties of the theory, and we defer to, e.g., ref. [34] for a more general discussion. ${ }^{4}$ The contribution to the integral from the boundary circle at infinity in $\mathcal{C}^{\prime}$ will vanish, leaving eq. (2.3) determined by the contribution above and below the discontinuities on the real $s$ axis. ${ }^{5}$ In detail, using $\operatorname{Disc} \mathcal{A}(s)=\lim _{\epsilon \rightarrow 0}[\mathcal{A}(s+i \epsilon)-\mathcal{A}(s-i \epsilon)]$, we have

$$
\frac{1}{2 \pi i} \oint_{\mathcal{C}^{\prime}} \frac{\mathrm{d} s}{s^{3}} \mathcal{A}(s)=\frac{1}{2 \pi i}\left(\int_{-\infty}^{-s_{\mathrm{d}}}+\int_{s_{\mathrm{d}}}^{\infty}\right) \frac{\mathrm{d} s}{s^{3}} \operatorname{Disc} \mathcal{A}(s)=\frac{1}{i \pi} \int_{s_{\mathrm{d}}}^{\infty} \frac{\mathrm{d} s}{s^{3}} \operatorname{Disc} \mathcal{A}(s)
$$

In the second equality in eq. (2.4), we sent $s \rightarrow-s$ in the first integral and subsequently invoked $1 \leftrightarrow 3$ crossing symmetry - assuming real polarizations so that the forward amplitude maps onto itself under crossing — which implies $\mathcal{A}(-s)=\mathcal{A}(s) .{ }^{6}$ Next, we can relate the discontinuity of the forward amplitude to the cross section:

$$
\operatorname{Disc} \mathcal{A}(s)=\lim _{\epsilon \rightarrow 0}[\mathcal{A}(s+i \epsilon)-\mathcal{A}(s-i \epsilon)]=\lim _{\epsilon \rightarrow 0}\left[\mathcal{A}(s+i \epsilon)-(\mathcal{A}(s+i \epsilon))^{*}\right]=2 i \operatorname{Im} \mathcal{A}(s),
$$

where we used the Schwarz reflection principle, $\mathcal{A}\left(s^{*}\right)=[\mathcal{A}(s)]^{*}$. Recalling the optical theorem $\operatorname{Im} \mathcal{A}(s)=s \sigma(s)$ (a consequence of unitarity), we find that

$$
\lambda_{2}=\frac{2}{\pi} \int_{s_{\mathrm{d}}}^{\infty} \frac{\mathrm{d} s}{s^{2}} \sigma(s) .
$$

\footnotetext{
${ }^{4}$ If the amplitude contains loops with massless particles, then branch cuts extend all the way to $s=0$. However, as in ref. [34] we can deform the theory in the IR by introducing a mass regulator, ensuring analyticity in a window near $s=0$, though subtleties arise in the case of gravity [40, 44].

${ }^{5}$ For massive states, the vanishing of the boundary integral at infinity is a consequence of the Froissart bound $[79,80]$. For massless states, this conclusion can instead be reached from the weak requirement that the amplitude of the UV completion scales more slowly with energy at large momentum than the EFT contribution [58].

${ }^{6}$ While the choice of real polarizations is not strictly necessary for placing positivity bounds [48], it will be convenient for our purposes.
} 
Finally, as cross sections are by definition positive, we conclude that $\lambda_{2}>0$. In the case of the scalar field example, $\lambda_{2}=4 c / M^{4}$, and so we have demonstrated that analyticity requires $c>0$.

Alternatively, we can arrive at $\lambda_{2}>0$ by invoking causality. Suppose $\lambda_{2}$ is the coefficient of an operator $\mathcal{O}\left[\phi_{1}, \phi_{2}\right]$, where $\mathcal{O}$ is quadratic in both $\phi_{1}$ and $\phi_{2}$ (here, $\phi_{1}$ and $\phi_{2}$ may or may not be distinct fields). Since $\lambda_{2}$ is defined to be the coefficient of $s^{2}$, there must be exactly four derivatives in $\mathcal{O}$. We wish to determine the dynamics of a perturbation in $\phi_{1}$ in a background of nonzero $\overline{\partial \phi_{2}}$ condensate. The two remaining derivatives in $\mathcal{O}$ will contribute to the dispersion relation quadratic in the momentum of $\phi_{1}$. Thus, if $\phi_{1}$ is massless, the speed $v$ of the $\phi_{1}$ perturbation is given schematically by

$$
v=1-\lambda_{2} \overline{\partial \phi_{2} \partial \phi_{2}}
$$

where as shown in refs. $[34,58,67]$ the signs in the speed calculation work out such that when $\lambda_{2}<0, v>1$. In that case, by taking two nonoverlapping bubbles of condensate, giving them a large relative boost (with relative speed $v_{\text {rel }}$ satisfying $1-v_{\text {rel }}=\mathcal{O}\left(\epsilon^{2}\right)$ when the signal speed is $v=1+\epsilon$ in the bubble [67]), and sending signals back and forth between the two bubbles [34], one can form a bona fide causal paradox, with the return signal arriving at the sender before the outgoing signal was sent [84, 85]; see figure 2 of ref. [34].

Let us demonstrate how this argument is realized in the simple scalar field example. Here, we have $\mathcal{O}\left[\phi_{1}, \phi_{2}\right]=(\partial \phi)^{4}$, so that $\phi_{1}=\phi_{2}=\phi$, and the operator is quartic in $\phi$ and $\partial$ as required. The equation of motion associated with eq. (2.1) is given by

$$
\square \phi-\frac{4 c}{M^{4}}\left[\square \phi(\partial \phi)^{2}+2\left(\partial^{\mu} \phi\right)\left(\partial^{\nu} \phi\right)\left(\partial_{\mu} \partial_{\nu} \phi\right)\right]=0 .
$$

In order to derive the bound, we must consider the dynamics of small perturbations in the scalar field around a nonzero background field. To do so, we set $\phi=\varphi+\bar{\phi}$, with $\varphi$ the perturbation we wish to study and $\bar{\phi}$ a background condensate chosen such that $\overline{\partial_{\mu} \phi}=q_{\mu}$, with $q_{\mu}$ a constant four-vector. The aim is to study the impact of interactions such as those in figure 3 on the propagation of $\varphi$. Working in this background, the linearized equation of motion for $\varphi$ becomes

$$
\left(-1+\frac{4 c q^{2}}{M^{4}}\right) \square \varphi+\frac{8 c}{M^{4}} q^{\mu} q^{\nu} \partial_{\mu} \partial_{\nu} \varphi=0 .
$$

If we expand $\varphi$ in plane waves, as $\varphi \propto e^{i k \cdot x}$, this becomes a dispersion relation

$$
\left(-1+\frac{4 c q^{2}}{M^{4}}\right) k^{2}+\frac{8 c}{M^{4}}(q \cdot k)^{2}=0
$$

Writing $k_{\mu}=\left(k_{0}, \mathbf{k}\right)$, the speed of propagation of $\varphi$ is $v=k_{0} /|\mathbf{k}|$, which we can compute from eq. (2.10) to be

$$
v=\sqrt{1-\frac{8 c(q \cdot k)^{2}}{|\mathbf{k}|^{2}\left(M^{4}-4 c q^{2}\right)}} \simeq 1-\frac{4 c(q \cdot k)^{2}}{M^{4} k_{0}^{2}},
$$




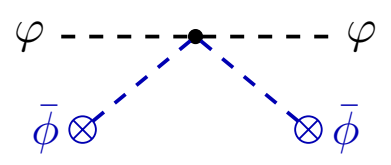

Figure 3. A representative interaction between a small perturbation $\varphi$ and the background scalar condensate $\bar{\phi}$ in which it is propagating. As shown in the text, in a theory where this interaction is mediated by a term $c(\partial \phi)^{4}$, unless $c>0$, these interactions will lead to the superluminal propagation of $\varphi$.

where in the second equality we expand to first order in the Wilson coefficient $c$. We conclude that unless $c>0, \varphi$ will experience superluminal propagation, allowing causal paradoxes to be constructed as described above.

\section{Operator basis}

Before proving our positivity bounds, let us first construct our basis of operators. As discussed in section 1, we wish to consider all operators in the SMEFT that are quartic in bosonic fields and that contain four derivatives. While the full set of operators at dimension eight in the SMEFT is vast [23, 24], containing 993 terms in a minimal basis, ${ }^{7}$ the scattering processes we consider will only activate a fraction of these operators, allowing us to tractably place positivity bounds.

\subsection{Four-derivative dimension-eight bosonic operators}

We will write the gauge field strengths for $\mathrm{U}(1)_{Y}$ weak hypercharge, $\mathrm{SU}(2)_{L}$ weak isospin, and $\mathrm{SU}(3)_{C}$ color as $B_{\mu \nu}, W_{\mu \nu}^{I}$, and $G_{\mu \nu}^{a}$, throughout using Greek letters for Lorentz indices, $I, J, K$ for weak indices, and $a, b, c, d$ for color indices. ${ }^{8}$ We define the dual field strengths via contraction with the Levi-Civita tensor $\epsilon^{\mu \nu \rho \sigma}: \widetilde{B}^{\mu \nu}=\epsilon^{\mu \nu \rho \sigma} B_{\rho \sigma} / 2, \widetilde{W}^{I \mu \nu}=\epsilon^{\mu \nu \rho \sigma} W_{\rho \sigma}^{I} / 2$, and $\widetilde{G}^{a \mu \nu}=\epsilon^{\mu \nu \rho \sigma} G_{\rho \sigma}^{a} / 2$.

A priori, we could construct arbitrary operators that are gauge and Lorentz singlets, contracting Lorentz indices with combinations of $g^{\mu \nu}$ and $\epsilon^{\mu \nu \rho \sigma}$ and gauge indices with $\delta^{I J}$ and $\epsilon^{I J K}$ for isospin and combinations of $\delta^{a b}, f^{a b c}$, and $d^{a b c}$ for color, where we define the structure constants via

$$
\begin{aligned}
{\left[T^{a}, T^{b}\right] } & =i f^{a b c} T^{c} \\
\left\{T^{a}, T^{b}\right\} & =\frac{1}{3} \delta^{a b} \mathbb{1}+d^{a b c} T^{c},
\end{aligned}
$$

for totally antisymmetric $f^{a b c}$, totally symmetric $d^{a b c}$, and defining the generators of SU(3) as $T^{a}=\lambda^{a} / 2$ in terms of the Gell-Mann matrices $\lambda^{a}$. However, many of these operators

\footnotetext{
${ }^{7}$ The figure of 993 results [23] from counting operators and hermitian conjugates separately, but not counting operators corresponding to different fermion generations. In this counting, there are 84 operators at dimension six, cf. ref. [22].

${ }^{8}$ Written in terms of the gauge fields $B_{\mu}, W_{\mu}^{I}$, and $G_{\mu}^{a}$, the field strengths are $B_{\mu \nu}=\partial_{\mu} B_{\nu}-\partial_{\nu} B_{\mu}$, $W_{\mu \nu}^{I}=\partial_{\mu} W_{\nu}^{I}-\partial_{\nu} W_{\mu}^{I}+g_{2} \epsilon^{I J K} W_{\mu}^{J} W_{\nu}^{K}$, and $G_{\mu \nu}^{a}=\partial_{\mu} G_{\nu}^{a}-\partial_{\nu} G_{\mu}^{a}+g_{3} f^{a b c} G_{\mu}^{b} G_{\nu}^{c}$. We write the gauge couplings of $\mathrm{U}(1)_{Y}, \mathrm{SU}(2)_{L}$, and $\mathrm{SU}(3)_{C}$ as $g_{1}, g_{2}$, and $g_{3}$, respectively.
} 
will not be linearly independent, by virtue of identities among Lorentz and color indices. The Levi-Civita identity, ${ }^{9}$

$$
\epsilon^{\mu \nu \rho \sigma} \epsilon_{\alpha \beta \gamma \delta}=-24 \delta_{\alpha}^{[\mu} \delta_{\beta}^{\nu} \delta_{\gamma}^{\rho} \delta_{\delta}^{\sigma]}
$$

implies, for example,

$$
B_{\mu \nu} B^{\nu \rho} B_{\rho \sigma} B^{\sigma \mu}=\frac{1}{2}\left(B_{\mu \nu} B^{\mu \nu}\right)^{2}+\frac{1}{4}\left(B_{\mu \nu} \widetilde{B}^{\mu \nu}\right)^{2} .
$$

The Schouten identity,

$$
\epsilon^{[\alpha \beta \gamma \delta} g^{\mu] \nu}=0,
$$

can also be used to reduce the list of operators, e.g.,

$$
4 \epsilon^{\mu \nu \rho \sigma} G_{\mu \alpha}^{a} G_{\nu}^{b \alpha} G_{\rho \beta}^{c} G_{\sigma}^{d \beta}=\left(G_{\mu \nu}^{a} G^{c \mu \nu} G_{\rho \sigma}^{b} \widetilde{G}^{d \rho \sigma}+G_{\mu \nu}^{b} G^{d \mu \nu} G_{\rho \sigma}^{a} \widetilde{G}^{c \rho \sigma}\right)-(c \leftrightarrow d) .
$$

To reduce the color structures, we exploit a particularly useful $\mathrm{SU}(N)$ identity,

$$
f^{a b e} f^{c d e}=\frac{2}{N}\left(\delta^{a c} \delta^{b d}-\delta^{a d} \delta^{b c}\right)+d^{a c e} d^{b d e}-d^{b c e} d^{a d e},
$$

and the Jacobi identity,

$$
f^{a b e} f^{e c d}+f^{c b e} f^{a e d}+f^{d b e} f^{a c e}=0,
$$

along with the additional $\mathrm{SU}(N)$ identities

$$
\begin{aligned}
f^{a b c} f^{a b d} & =N \delta^{c d} \\
d^{a b c} d^{a b d} & =\frac{N^{2}-4}{N} \delta^{c d} \\
f^{a b e} d^{c d e}+f^{a c e} d^{b d e}+f^{a d e} d^{b c e} & =0 \\
f^{a b e} d^{c d e}+f^{c b e} d^{a d e}+f^{d b e} d^{a c e} & =0 .
\end{aligned}
$$

Moreover, for SU(3), there are two additional identities:

$$
\begin{aligned}
3 d^{a b e} d^{c d e}-f^{a c e} f^{b d e}-f^{a d e} f^{b c e} & =\delta^{a c} \delta^{b d}+\delta^{a d} \delta^{b c}-\delta^{a b} \delta^{c d} \\
3\left(d^{a b e} d^{c d e}+d^{a c e} d^{b d e}+d^{a d e} d^{b c e}\right) & =\delta^{a b} \delta^{c d}+\delta^{a c} \delta^{b d}+\delta^{a d} \delta^{b c} .
\end{aligned}
$$

Using these relations, one can construct a minimal basis of operators quartic in the field strength for Yang-Mills theory, as originally derived in ref. [86]. In particular, one can show that, among the $G^{4}$-type operators quartic in the gluon field strength of $\mathrm{SU}(3)_{C}$, the minimal basis consists of $6 \mathrm{CP}$-conserving and $3 \mathrm{CP}$-violating operators. Analogously, for the $W^{4}$ operators of $\mathrm{SU}(2)_{L}$, since $d^{a b c}$ vanishes for this group, the minimal basis comprises $4 \mathrm{CP}$-conserving and $2 \mathrm{CP}$-violating operators, while for the $B^{4}$ operators of $\mathrm{U}(1)$, there

\footnotetext{
${ }^{9}$ We denote normalized symmetrization via $\lambda^{\left(a_{1} \cdots a_{n}\right)}=\frac{1}{n !}\left(\lambda^{a_{1} \cdots a_{n}}+\right.$ permutations $)$ and antisymmetrization similarly with $\left[a_{1} \cdots a_{n}\right]$.
} 
are 2 independent $\mathrm{CP}$-conserving operators and $1 \mathrm{CP}$-violating operator. The minimal basis is given in table 1 .

We can similarly construct a minimal basis of cross-quartic operators. For operators of the $B^{2} W^{2}$ form, there are $4 \mathrm{CP}$-conserving and $3 \mathrm{CP}$-violating independent operators, and similarly for operators of the $B^{2} G^{2}$ and $W^{2} G^{2}$ types. Moreover, there are 2 independent $\mathrm{CP}$-conserving and $2 \mathrm{CP}$-violating operators of the $B G^{3}$ form (but there are no nonvanishing operators of the $B W^{3}$ form). All of these operators are also given in table 1 .

Finally, we define the complex Higgs field $H$ as a fundamental $(2)$ of $\mathrm{SU}(2)_{L}$, which we can write in terms of four real scalar fields $\phi_{1,2,3,4}$ as

$$
H_{i}=\frac{1}{\sqrt{2}}\left(\begin{array}{c}
\phi_{1}+i \phi_{2} \\
\phi_{3}+i \phi_{4}
\end{array}\right),
$$

with conjugate

$$
H_{i}^{\dagger}=\frac{1}{\sqrt{2}}\left(\begin{array}{c}
-\phi_{3}+i \phi_{4} \\
\phi_{1}-i \phi_{2}
\end{array}\right) .
$$

We contract fundamental $\mathrm{SU}(2)$ indices in two different ways. First, contractions of the form $H^{\dagger} H$ are performed using $\epsilon_{i j} .{ }^{10}$ On occasion, we will also express the triplet resulting from the product of two doublets as, for example, $H^{\dagger} \tau^{K} H=H^{\dagger i}\left(\tau^{K}\right)_{i}^{j} H_{j}$, where $\tau^{I}=\sigma^{I} / 2$, with $\sigma^{I}$ the usual Pauli matrices. Further, we define the covariant derivative $D_{\mu} H=\left(\partial_{\mu}+\frac{1}{2} i g_{1} B_{\mu}+i g_{2} \tau^{I} W_{\mu}^{I}\right) H$. Then, as shown by ref. [87], there are three linearlyindependent operators of the form $(D H)^{4}$, as well as $10 \mathrm{CP}$-conserving and $8 \mathrm{CP}$-violating four-derivative bosonic cross-quartics containing the Higgs; see table $2 .{ }^{11}$

In total, the basis in tables 1 and 2 contains 64 terms, of which 39 conserve and 25 violate CP. All possible quartic, four-derivative, Lorentz and gauge invariant dimensioneight operators in the SMEFT can be written as a linear combination of operators in this basis. We will write the SMEFT action relevant for our bounds as

$$
\begin{aligned}
\mathcal{L}= & -\frac{1}{4} B_{\mu \nu} B^{\mu \nu}-\frac{1}{4} W_{\mu \nu}^{I} W^{I \mu \nu}-\frac{1}{4} G_{\mu \nu}^{a} G^{a \mu \nu}-\left(D^{\mu} H\right)^{\dagger} D_{\mu} H \\
& -\mu^{2} H^{\dagger} H+\lambda\left(H^{\dagger} H\right)^{2}+\frac{1}{M^{4}} \sum_{i} c_{i} \mathcal{O}_{i},
\end{aligned}
$$

where $M$ is the scale of new physics, the sum over the $\mathcal{O}_{i}$ contains all the operators in tables 1 and 2 (e.g., $\mathcal{O}_{1}^{G^{4}}, \mathcal{O}_{2}^{H^{2} G^{2}}, \widetilde{\mathcal{O}}_{3}^{B^{2}} W^{2}$, etc.), and the $c_{i}$ are unitless couplings decorated analogously with their corresponding operators (e.g., $c_{1}^{G^{4}}, c_{2}^{H^{2} G^{2}}, \widetilde{c}_{3}^{B^{2} W^{2}}$, etc.). In all tables, each operator in the basis is self-hermitian, so the $c_{i}$ are real. It is on this set of couplings $c_{i}$ that we will derive bounds from analyticity and causality, which will be the main result of this work.

\footnotetext{
${ }^{10}$ We follow the convention of ref. [87], treating $H_{i}^{\dagger}=\epsilon_{i j} H^{\dagger j}$ as a $\mathbf{2}$ of $\mathrm{SU}(2)$ rather than $\overline{\mathbf{2}}$. Here $i$ indexes the fundamental representation of $\mathrm{SU}(2)$ and runs over $i \in\{1,2\}$. We define $\epsilon^{12}=\epsilon_{21}=+1$, so that indices are contracted as $H_{i}=\epsilon_{i j} H^{j}, H^{i}=\epsilon^{i j} H_{j}$, and $H^{\dagger} H=\epsilon^{i j} H_{j}^{\dagger} H_{i}=+\left(\phi_{1}^{2}+\phi_{2}^{2}+\phi_{3}^{2}+\phi_{4}^{2}\right) / 2$.

${ }^{11}$ As confirmed by ref. [88], we note that ref. [87] contains a typo in the equivalent expressions for our $\mathcal{O}_{3}^{H^{2} W^{2}}, \widetilde{\mathcal{O}}_{3}^{H^{2} W^{2}}$, and $\widetilde{\mathcal{O}}_{2}^{H^{2}} B W$. In ref. [87], the equivalent expressions are missing a factor of $i$ required to make them self-hermitian. (In detail, the relevant terms in their notation are $\mathcal{O}_{8, D H W 3}, \mathcal{O}_{8, D H W 3 b}$, and $\mathcal{O}_{8, D H \tilde{W} B 2}$ in their table 5.)
} 


\begin{tabular}{|c|c|c|c|}
\hline & $B^{4}$ operators & \multicolumn{2}{|c|}{$F_{1}^{2} F_{2}^{2} / F_{1} F_{2}^{3}$ cross-quartics } \\
\hline $\mathcal{O}_{1}^{B^{4}}$ & $(B B)(B B)$ & $\mathcal{O}_{1}^{B^{2} W^{2}}$ & $(B B)\left(W^{I} W^{I}\right)$ \\
\hline $\mathcal{O}_{2}^{B^{4}}$ & $(B \widetilde{B})(B \widetilde{B})$ & $\mathcal{O}_{2}^{B^{2} W^{2}}$ & $(B \widetilde{B})\left(W^{I} \widetilde{W}^{I}\right)$ \\
\hline \multirow{5}{*}{$\widetilde{\mathcal{O}}_{1}^{B^{4}}$} & $(B B)(B \widetilde{B})$ & $\mathcal{O}_{3}^{B^{2} W^{2}}$ & $\left(B W^{I}\right)\left(B W^{I}\right)$ \\
\hline & & $\mathcal{O}_{4}^{B^{2} W^{2}}$ & $\left(B \widetilde{W}^{I}\right)\left(B \widetilde{W}^{I}\right)$ \\
\hline & & $\widetilde{\mathcal{O}}_{1}^{B^{2} W^{2}}$ & $(B \widetilde{B})\left(W^{I} W^{I}\right)$ \\
\hline & $W^{4}$ operators & $\widetilde{\mathcal{O}}_{2}^{B^{2} W^{2}}$ & $(B B)\left(W^{I} \widetilde{W}^{I}\right)$ \\
\hline & & $\widetilde{\mathcal{O}}_{3}^{B^{2} W^{2}}$ & $\left(B W^{I}\right)\left(B \widetilde{W}^{I}\right)$ \\
\hline \multicolumn{4}{|c|}{$\left(W^{I} W^{I}\right)\left(W^{J} W^{J}\right)$} \\
\hline $\mathcal{O}_{2}^{W^{4}}$ & $\left(W^{I} \widetilde{W}^{I}\right)\left(W^{J} \widetilde{W}^{J}\right)$ & $\mathcal{O}_{1}^{B^{2} G^{2}}$ & $(B B)\left(G^{a} G^{a}\right)$ \\
\hline $\mathcal{O}_{3}^{W^{4}}$ & $\left(W^{I} W^{J}\right)\left(W^{I} W^{J}\right)$ & $\mathcal{O}_{2}^{B^{2} G^{2}}$ & $(B \widetilde{B})\left(G^{a} \widetilde{G}^{a}\right)$ \\
\hline $\mathcal{O}_{4}^{W^{4}}$ & $\left(W^{I} \widetilde{W}^{J}\right)\left(W^{I} \widetilde{W}^{J}\right)$ & $\mathcal{O}_{3}^{B^{2} G^{2}}$ & $\left(B G^{a}\right)\left(B G^{a}\right)$ \\
\hline$\widetilde{O}_{1}^{W^{4}}$ & $\left(W^{I} W^{I}\right)\left(W^{J} \widetilde{W}^{J}\right)$ & $\mathcal{O}_{4}^{B^{2} G^{2}}$ & $\left(B \widetilde{G}^{a}\right)\left(B \widetilde{G}^{a}\right)$ \\
\hline \multirow[t]{5}{*}{$\widetilde{\mathcal{O}}_{2}^{W^{4}}$} & $\left(W^{I} W^{J}\right)\left(W^{I} \widetilde{W}^{J}\right)$ & $\widetilde{\mathcal{O}}_{1}^{B^{2} G^{2}}$ & $(B \widetilde{B})\left(G^{a} G^{a}\right)$ \\
\hline & & $\widetilde{\mathcal{O}}_{2}^{B^{2} G^{2}}$ & $(B B)\left(G^{a} \widetilde{G}^{a}\right)$ \\
\hline & & $\widetilde{\mathcal{O}}_{3}^{B^{2} G^{2}}$ & $\left(B G^{a}\right)\left(B \widetilde{G}^{a}\right)$ \\
\hline & $G^{4}$ operators & & \\
\hline & & $\mathcal{O}_{1}^{W^{2} G^{2}}$ & $\left(W^{I} W^{I}\right)\left(G^{a} G^{a}\right)$ \\
\hline $\mathcal{O}_{1}^{G^{4}}$ & $\left(G^{a} G^{a}\right)\left(G^{b} G^{b}\right)$ & $\mathcal{O}_{2}^{W^{2} G^{2}}$ & $\left(W^{I} \widetilde{W}^{I}\right)\left(G^{a} \widetilde{G}^{a}\right)$ \\
\hline \multirow{2}{*}{$\begin{array}{l}\mathcal{O}_{2}^{G^{4}} \\
\mathcal{O}_{3}^{G^{4}}\end{array}$} & $\left(G^{a} \widetilde{G}^{a}\right)\left(G^{b} \widetilde{G}^{b}\right)$ & $\mathcal{O}_{3}^{W^{2} G^{2}}$ & $\left(W^{I} G^{a}\right)\left(W^{I} G^{a}\right)$ \\
\hline & $\left(G^{a} G^{b}\right)\left(G^{a} G^{b}\right)$ & $\mathcal{O}_{4}^{W^{2} G^{2}}$ & $\left(W^{I} \widetilde{G}^{a}\right)\left(W^{I} \widetilde{G}^{a}\right)$ \\
\hline \multirow{2}{*}{$\begin{array}{l}\mathcal{O}_{4}^{G^{4}} \\
\mathcal{O}_{5}^{G^{4}}\end{array}$} & $\left(G^{a} \widetilde{G}^{b}\right)\left(G^{a} \widetilde{G}^{b}\right)$ & $\widetilde{\mathcal{O}}_{1}^{W^{2} G^{2}}$ & $\left(W^{I} \widetilde{W}^{I}\right)\left(G^{a} G^{a}\right)$ \\
\hline & $d^{a b e} d^{c d e}\left(G^{a} G^{b}\right)\left(G^{c} G^{d}\right)$ & $\widetilde{\mathcal{O}}_{2}^{W^{2} G^{2}}$ & $\left(W^{I} W^{I}\right)\left(G^{a} \widetilde{G}^{a}\right)$ \\
\hline \multirow{2}{*}{$\begin{array}{l}\mathcal{O}_{6}^{G^{4}} \\
\widetilde{O}_{1}^{G^{4}}\end{array}$} & $d^{a b e} d^{c d e}\left(G^{a} \widetilde{G}^{b}\right)\left(G^{c} \widetilde{G}^{d}\right)$ & $\widetilde{\mathcal{O}}_{3}^{W^{2} G^{2}}$ & $\left(W^{I} G^{a}\right)\left(W^{I} \widetilde{G}^{a}\right)$ \\
\hline & $\left(G^{a} G^{a}\right)\left(G^{b} \widetilde{G}^{b}\right)$ & & \\
\hline \multirow{4}{*}{$\begin{array}{l}\widetilde{\mathcal{O}}_{2}^{G^{4}} \\
\widetilde{\mathcal{O}}_{3}^{G^{4}}\end{array}$} & $\left(G^{a} G^{b}\right)\left(G^{a} \widetilde{G}^{b}\right)$ & $\mathcal{O}_{1}^{B G^{3}}$ & $d^{a b c}\left(B G^{a}\right)\left(G^{b} G^{c}\right)$ \\
\hline & $d^{a b e} d^{c d e}\left(G^{a} G^{b}\right)\left(G^{c} \widetilde{G}^{d}\right)$ & $\mathcal{O}_{2}^{B G^{3}}$ & $d^{a b c}\left(B \widetilde{G}^{a}\right)\left(G^{b} \widetilde{G}^{c}\right)$ \\
\hline & & $\widetilde{\mathcal{O}}_{1}^{B G^{3}}$ & $d^{a b c}\left(B \widetilde{G}^{a}\right)\left(G^{b} G^{c}\right)$ \\
\hline & & $\widetilde{\mathcal{O}}_{2}^{B G^{3}}$ & $d^{a b c}\left(B G^{a}\right)\left(G^{b} \widetilde{G}^{c}\right)$ \\
\hline
\end{tabular}

Table 1. Basis of independent dimension-eight operators in the SMEFT that are quartic in gauge field strengths. CP-violating operators are denoted with a tilde. Throughout the table, we have for clarity suppressed Lorentz indices, writing $(A B)$ for $A_{\mu \nu} B^{\mu \nu}$. 


\begin{tabular}{|c|c|}
\hline \multicolumn{2}{|r|}{$(D H)^{4}$ operators } \\
\hline $\mathcal{O}_{1}^{H^{4}}$ & $\left(D_{\mu} H^{\dagger} D_{\nu} H\right)\left(D^{\nu} H^{\dagger} D^{\mu} H\right)$ \\
\hline $\mathcal{O}_{2}^{H^{4}}$ & $\left(D_{\mu} H^{\dagger} D_{\nu} H\right)\left(D^{\mu} H^{\dagger} D^{\nu} H\right)$ \\
\hline $\mathcal{O}_{3}^{H^{4}}$ & $\left(D^{\mu} H^{\dagger} D_{\mu} H\right)\left(D^{\nu} H^{\dagger} D_{\nu} H\right)$ \\
\hline \multicolumn{2}{|r|}{$(D H)^{2} F^{2}$ cross-quartics } \\
\hline $\mathcal{O}_{1}^{H^{2} B^{2}}$ & $\left(D^{\mu} H^{\dagger} D^{\nu} H\right) B_{\mu \rho} B_{\nu}{ }^{\rho}$ \\
\hline $\mathcal{O}_{2}^{H^{2} B^{2}}$ & $\left(D^{\mu} H^{\dagger} D_{\mu} H\right) B_{\rho \sigma} B^{\rho \sigma}$ \\
\hline$\widetilde{\mathcal{O}}_{1}^{H^{2} B^{2}}$ & $\left(D^{\mu} H^{\dagger} D_{\mu} H\right) B_{\rho \sigma} \widetilde{B}^{\rho \sigma}$ \\
\hline $\mathcal{O}_{1}^{H^{2} W^{2}}$ & $\left(D^{\mu} H^{\dagger} D^{\nu} H\right) W_{\mu \rho}^{I} W_{\nu}^{I \rho}$ \\
\hline $\mathcal{O}_{2}^{H^{2} W^{2}}$ & $\left(D^{\mu} H^{\dagger} D_{\mu} H\right) W_{\rho \sigma}^{I} W^{I \rho \sigma}$ \\
\hline $\mathcal{O}_{3}^{H^{2} W^{2}}$ & $i \epsilon^{I J K}\left(D^{\mu} H^{\dagger} \tau^{I} D^{\nu} H\right) W_{\mu \rho}^{J} W_{\nu}^{K \rho}$ \\
\hline$\widetilde{\mathcal{O}}_{1}^{H^{2}} W^{2}$ & $\left(D^{\mu} H^{\dagger} D_{\mu} H\right) W_{\rho \sigma}^{I} \widetilde{W}^{I \rho \sigma}$ \\
\hline$\widetilde{\mathcal{O}}_{2}^{H^{2}} W^{2}$ & $\epsilon^{I J K}\left(D^{\mu} H^{\dagger} \tau^{I} D^{\nu} H\right)\left(W_{\mu \rho}^{J} \widetilde{W}_{\nu}^{K \rho}-\widetilde{W}_{\mu \rho}^{J} W_{\nu}^{K \rho}\right)$ \\
\hline$\widetilde{\mathcal{O}}_{3}^{H^{2}} W^{2}$ & $i \epsilon^{I J K}\left(D^{\mu} H^{\dagger} \tau^{I} D^{\nu} H\right)\left(W_{\mu \rho}^{J} \widetilde{W}_{\nu}^{K \rho}+\widetilde{W}_{\mu \rho}^{J} W_{\nu}^{K \rho}\right)$ \\
\hline $\mathcal{O}_{1}^{H^{2} G^{2}}$ & $\left(D^{\mu} H^{\dagger} D^{\nu} H\right) G_{\mu \rho}^{a} G_{\nu}^{a \rho}$ \\
\hline $\mathcal{O}_{2}^{H^{2} G^{2}}$ & $\left(D^{\mu} H^{\dagger} D_{\mu} H\right) G_{\rho \sigma}^{a} G^{a \rho \sigma}$ \\
\hline$\widetilde{\mathcal{O}}_{1}^{H^{2} G^{2}}$ & $\left(D^{\mu} H^{\dagger} D_{\mu} H\right) G_{\rho \sigma}^{a} \widetilde{G}^{a \rho \sigma}$ \\
\hline \multicolumn{2}{|r|}{$(D H)^{2} F_{1} F_{2}$ cross-quartics } \\
\hline $\mathcal{O}_{1}^{H^{2} B W}$ & $\left(D^{\mu} H^{\dagger} \tau^{I} D_{\mu} H\right) B_{\rho \sigma} W^{I \rho \sigma}$ \\
\hline $\mathcal{O}_{2}^{H^{2} B W}$ & $i\left(D^{\mu} H^{\dagger} \tau^{I} D^{\nu} H\right)\left(B_{\mu \rho} W_{\nu}^{I \rho}-B_{\nu \rho} W_{\mu}^{I \rho}\right)$ \\
\hline $\mathcal{O}_{3}^{H^{2} B W}$ & $\left(D^{\mu} H^{\dagger} \tau^{I} D^{\nu} H\right)\left(B_{\mu \rho} W_{\nu}^{I \rho}+B_{\nu \rho} W_{\mu}^{I \rho}\right)$ \\
\hline$\widetilde{\mathcal{O}}_{1}^{H^{2} B W}$ & $\left(D^{\mu} H^{\dagger} \tau^{I} D_{\mu} H\right) B_{\rho \sigma} \widetilde{W}^{I \rho \sigma}$ \\
\hline$\widetilde{\mathcal{O}}_{2}^{H^{2}} B W$ & $i\left(D^{\mu} H^{\dagger} \tau^{I} D^{\nu} H\right)\left(B_{\rho[\mu} \widetilde{W}_{\nu]}^{I \rho}-\widetilde{B}_{\rho[\mu} W_{\nu]}^{I \rho}\right)$ \\
\hline$\widetilde{\mathcal{O}}_{3}^{H^{2}} B W$ & $\left(D^{\mu} H^{\dagger} \tau^{I} D^{\nu} H\right)\left(B_{\rho(\mu} \widetilde{W}_{\nu)}^{I \rho}+\widetilde{B}_{\rho(\mu} W_{\nu)}^{I \rho}\right)$ \\
\hline
\end{tabular}

Table 2. Basis of independent dimension-eight operators in the SMEFT that are quartic in bosons and contain four derivatives and/or field strengths and at least one Higgs. CP-violating operators are denoted with a tilde. Factors of $i$ ensure that each operator is self-hermitian and thus has real Wilson coefficient. 


\begin{tabular}{|ll|ll|}
\hline $\mathcal{O}_{\operatorname{dim}-6}^{H^{2} B^{2}}$ & $H^{\dagger} H B_{\mu \nu} B^{\mu \nu}$ & $\mathcal{O}_{\operatorname{dim}-6}^{W^{3}}$ & $\epsilon^{I J K} W_{\mu}^{I \nu} W_{\nu}^{J \rho} W_{\rho}^{K \mu}$ \\
$\widetilde{\mathcal{O}}_{\operatorname{dim}-6}^{H^{2} B^{2}}$ & $H^{\dagger} H B_{\mu \nu} \widetilde{B}^{\mu \nu}$ & $\widetilde{\mathcal{O}}_{\operatorname{dim}-6}^{W^{3}}$ & $\epsilon^{I J K} W_{\mu}^{I \nu} W_{\nu}^{J \rho} \widetilde{W}_{\rho}^{K \mu}$ \\
$\mathcal{O}_{\operatorname{dim}}^{H^{2} W^{2}}$ & $H^{\dagger} H W_{\mu \nu}^{I} W^{I \mu \nu}$ & $\mathcal{O}_{\operatorname{dim}-6}^{G^{3}}$ & $f^{a b c} G_{\mu}^{a \nu} G_{\nu}^{b \rho} G_{\rho}^{c \mu}$ \\
$\widetilde{\mathcal{O}}_{\operatorname{dim}-6}^{H^{2} W^{2}}$ & $H^{\dagger} H W_{\mu \nu}^{I} \widetilde{W}^{I \mu \nu}$ & $\widetilde{\mathcal{O}}_{\operatorname{dim}-6}^{G^{3}}$ & $f^{a b c} G_{\mu}^{a \nu} G_{\nu}^{b \rho} \widetilde{G}_{\rho}^{c \mu}$ \\
$\mathcal{O}_{\operatorname{dim}-6}^{H^{2} G^{2}}$ & $H^{\dagger} H G_{\mu \nu}^{a} G^{a \mu \nu}$ & $\mathcal{O}_{1, \operatorname{dim}-6}^{H^{4}}$ & $\left(H^{\dagger} H\right) \square\left(H^{\dagger} H\right)$ \\
$\widetilde{\mathcal{O}}_{\operatorname{dim}-6}^{H^{2} G^{2}}$ & $H^{\dagger} H G_{\mu \nu}^{a} \widetilde{G}^{a \mu \nu}$ & $\mathcal{O}_{2, \operatorname{dim}-6}^{H^{4}}$ & $\left(H^{\dagger} D^{\mu} H\right)^{\star}\left(H^{\dagger} D_{\mu} H\right)$ \\
$\mathcal{O}_{\operatorname{dim}-6}^{H^{2} B W}$ & $H^{\dagger} \tau^{I} H B_{\mu \nu} W^{I \mu \nu}$ & $\mathcal{O}_{\operatorname{dim}-6}^{H^{6}}$ & $\left(H^{\dagger} H\right)^{3}$ \\
$\widetilde{\mathcal{O}}_{\operatorname{dim}-6}^{H^{2} B W}$ & $H^{\dagger} \tau^{I} H B_{\mu \nu} \widetilde{W}^{I \mu \nu}$ & & \\
\hline
\end{tabular}

Table 3. Bosonic operators at dimension six in the SMEFT.

\subsection{Lower-dimension operators}

In a general EFT of the SM, there are additional higher-dimension operators appearing at mass dimension five, six, and seven. For a general scattering process or perturbative calculation, such operators are formally dominant over dimension-eight terms. However, for the four-boson operators we will consider, we can exclude such operators from consideration as follows.

The only dimension-five operator in the SMEFT is the Weinberg operator [89], which generates Majorana neutrino masses. This operator contains fermionic fields, but we will be considering four-point scattering of bosons and the propagation of bosonic states in backgrounds that have nonzero bosonic field support but vanishing classical fermionic field backgrounds. That is, since fermion lines cannot end in Feynman diagrams, fermions cannot contribute to four-point boson scattering at leading order in perturbation theory. Hence, the dimension-five term's contribution to the amplitude in the low-energy EFT can be neglected for the processes we consider. By the same token, since all dimension-seven operators in the SMEFT contain fermions [23], we can exclude these from our analysis as well.

The SMEFT at dimension six is well studied; see refs. [22, 23, 89-95] and refs. therein. The operator basis, given in ref. [22], contains 59 independent baryon number conserving and 4 baryon number violating operators (counting hermitian conjugates as part of the original operator). Of these terms, 48 contain fermions, which we exclude by the reasoning given above, leaving the 15 purely bosonic operators given in table 3 .

Since the quartic operators with a Higgs in table 3 - of the schematic form $H^{2} F^{2}$, $H^{2} \square H^{2}$, or $(H D H)^{2}$ — have terms containing at most two derivatives, a single insertion of these operators contributes at lower order in momenta in the four-point amplitude; hence, such terms will give no contribution to the $s^{2}$ coefficient in the forward amplitude and will thus drop out of the contour integral in eq. (2.3) by the residue theorem. Again, we need not consider multiple insertions of quartic operators, since we are computing amplitudes to leading order low-energy EFT. In the causality calculation, such operators also do not contribute to the first-order change to the speed of propagation, since the two derivatives in 
these operators become two insertions of the zeroth-order momentum $k$, and one can see by table 3 that these two $k$ momenta will always both be contracted into each other (yielding zero contribution to the first-order dispersion relation), into a polarization (yielding zero by the Lorenz gauge condition), or into a Levi-Civita tensor (again yielding zero). Hence, we can drop all the operators in the first column of table 3 , as well as $\mathcal{O}_{1, \mathrm{dim}-6}^{H^{4}}$ and $\mathcal{O}_{2 \text {,dim- } 6 \text {. }}^{H^{4}}$. We can also drop the six-point term $\mathcal{O}_{\mathrm{dim}-6}^{H^{6}}$, since it cannot contribute to the leading-order four-point amplitudes in the EFT.

We are thus left with only four operators to consider at dimension six: $\mathcal{O}_{\text {dim-6 }}^{W^{3}}, \widetilde{\mathcal{O}}_{\text {dim-6 }}^{W^{3}}$, $\mathcal{O}_{\text {dim-6 } 6}^{G^{3}}$, and $\widetilde{\mathcal{O}}_{\text {dim- } 6}^{G^{3}}$. Expanding in gauge fields, these operators can generate both cubic and quartic vertices, which can contribute to four-point $W$ boson or gluon scattering. While a single insertion of the quartic will be of lower order in derivatives, exchange of a gauge boson through two insertions of the dimension-six operator will give a contribution independent of the sign of the coupling and at the same order in derivatives as a single insertion of a four-point dimension-eight vertex, thus obstructing the bounds we wish to place. We can avoid this obstacle by simply choosing to scatter incoming states with commuting colors. Writing $u_{1}^{a}$ and $u_{2}^{a}$ for the two color vectors of the incoming gluons (and similarly for the $W$ bosons), choosing $u_{1,2}$ such that

$$
f^{a b c} u_{1}^{b} u_{2}^{c}=0
$$

guarantees that the leading-order four-point forward amplitude induced by $\mathcal{O}_{\operatorname{dim}-6}^{G^{3}}$, and $\widetilde{\mathcal{O}}_{\text {dim-6 } 6}^{G^{3}}$ vanishes. Similarly, choosing eq. (3.13), where now $u_{1,2}$ are the colors of the background and perturbation, guarantees that these operators do not change the speed of propagation. A similar conclusion holds for the $W^{3}$ operators. The requirement in eq. (3.13) also excises the $t$-channel singularity, which scales as $s / t$, from the forward Yang-Mills amplitude. By requiring states with commuting colors according to eq. (3.13), we are choosing gluons within the abelian Cartan subalgebra describing the diagonal generators of $\mathrm{SU}(N)$; the Cartan subalgebra has $N-1$ generators, so we will derive $N-1 \mathrm{U}(1)$-like bounds from $\mathrm{SU}(N)$ scattering.

Finally, we should consider whether contributions from the unperturbed SM itself interfere with our bounds. Given the paucity of direct new physics detections at the LHC and elsewhere, we make the well-motivated assumption that the scale $M$ of new physics is above the weak scale. We can therefore treat all of the bosons in our thought experiments as massless to good approximation. Above the weak scale, the SM obeys perturbative unitarity, so amplitudes from purely SM operators are well behaved at large momenta. In contrast, the addition of four-derivative (dimension-eight) EFT operators induces amplitudes that diverge $s^{2}$. As before, since the contour integral in $s$ extracts the $s^{2}$ part of the amplitude, we can ignore at leading order any contribution from the uncorrected SM. Accordingly, in all amplitudes we subsequently write, we will suppress these contributions, retaining only the terms induced by the operators in tables 1 and 2. Moreover, the condition in eq. (3.13) (along with its $\mathrm{SU}(2)$ analogue) ensures that a gauge field background of one color does not interfere with the null propagation of gauge fields of the other color, and similarly the Yang-Mills contribution to the forward amplitude vanishes. 


\begin{tabular}{|ll|}
\hline $\mathcal{O}_{1}^{F^{4}}$ & $\left(F^{a} F^{a}\right)\left(F^{b} F^{b}\right)$ \\
$\mathcal{O}_{2}^{F^{4}}$ & $\left(F^{a} \widetilde{F}^{a}\right)\left(F^{b} \widetilde{F}^{b}\right)$ \\
$\mathcal{O}_{3}^{F^{4}}$ & $\left(F^{a} F^{b}\right)\left(F^{a} F^{b}\right)$ \\
$\mathcal{O}_{4}^{F^{4}}$ & $\left(F^{a} \widetilde{F}^{b}\right)\left(F^{a} \widetilde{F}^{b}\right)$ \\
$\mathcal{O}_{5}^{F^{4}}$ & $d^{a b e} d^{c d e}\left(F^{a} F^{b}\right)\left(F^{c} F^{d}\right)$ \\
$\mathcal{O}_{6}^{F^{4}}$ & $d^{a b e} d^{c d e}\left(F^{a} \widetilde{F}^{b}\right)\left(F^{c} \widetilde{F}^{d}\right)$ \\
$\mathcal{O}_{7}^{F^{4}}$ & $d^{a c e} d^{b d e}\left(F^{a} F^{b}\right)\left(F^{c} F^{d}\right)$ \\
$\mathcal{O}_{8}^{F^{4}}$ & $d^{a c e} d^{b d e}\left(F^{a} \widetilde{F}^{b}\right)\left(F^{c} \widetilde{F}^{d}\right)$ \\
$\widetilde{\mathcal{O}}_{1}^{F^{4}}$ & $\left(F^{a} F^{a}\right)\left(F^{b} \widetilde{F}^{b}\right)$ \\
$\widetilde{\mathcal{O}}_{2}^{F^{4}}$ & $\left(F^{a} F^{b}\right)\left(F^{a} \widetilde{F}^{b}\right)$ \\
$\widetilde{\mathcal{O}}_{3}^{F^{4}}$ & $d^{a b e} d^{c d e}\left(F^{a} F^{b}\right)\left(F^{c} \widetilde{F}^{d}\right)$ \\
$\widetilde{\mathcal{O}}_{4}^{F^{4}}$ & $d^{a c e} d^{b d e}\left(F^{a} F^{b}\right)\left(F^{c} \widetilde{F}^{d}\right)$ \\
\hline
\end{tabular}

Table 4. Basis of independent operators for $\mathrm{SU}(N)$ Yang-Mills theory that are quartic in gauge field strengths. Notation is as in table 1.

Thus, provided we take $M$ above the weak scale and choose colors and isospins according to eq. (3.13), we are able to apply the analyticity and causality arguments reviewed in section 2 to four-point contact terms in the EFT in order to bound the dimension-eight operators without being affected by operators of lower mass dimension.

\section{Bounds}

Using the causality and analyticity techniques of section 2, we now derive bounds on the EFT coefficients of the operators in tables 1 and 2 . In total we will derive 27 independent bounds, and we will do so by considering different classes of operators separately.

\subsection{Single field strength quartics}

Let us first consider bounding the operators in table 1 that contain a single type of gauge field. It will be useful to first generalize to gauge group $\mathrm{SU}(N)$ for $N$ arbitrary, writing the gauge field as $A_{\mu}^{a}$ and field strength at $F_{\mu \nu}^{a}$. As shown in ref. [86], there are eight $\mathrm{CP}$-conserving and four CP-violating operators of the form $F^{4}$, given in table 4; the larger number of operators, compared to the $\mathrm{SU}(3)$ operators in table 1, is due to the fact that the identities in eq. (3.9) do not apply for general $N$.

We first consider the constraint that causality imposes on this more general set of operators. Taking the Lagrangian $-\frac{1}{4} F_{\mu \nu}^{a} F^{a \mu \nu}+\frac{1}{M^{4}} \sum_{i} c_{i} \mathcal{O}_{i}$, for the $\mathcal{O}_{i}$ in table 4 , the leading-order (i.e., uncorrected Yang-Mills) equation of motion is

$$
D^{\mu} F_{\mu \nu}^{a}=\partial^{\mu} F_{\mu \nu}^{a}+g f^{a b c} A^{\mu b} F_{\mu \nu}^{c}=0,
$$

where $g$ is the gauge coupling. This equation of motion is solved by a constant $F_{\mu \nu}^{a}$, so we take the background gauge field to be

$$
\bar{A}_{\mu}^{a}=u_{1}^{a} \epsilon_{1 \mu} w,
$$


where $u_{1}$ is a constant real vector in color space, $\epsilon_{1}$ is a constant four-vector of arbitrary signature, and $w$ is an arbitrary Cartesian coordinate in spacetime, i.e., $\partial_{\mu} w=\ell_{\mu}$, where $\ell_{\mu}$ is a constant four-vector; we make no assumptions regarding the direction or signature of $\ell$ or $\epsilon_{1} \cdot{ }^{12}$ Note that $u_{1}$ is not a generator of $\mathrm{SU}(N)$; $u_{1}$ is not matrix-valued, but is simply a constant vector of real numbers. We then expand around this background, writing $A_{\mu}^{a}=\bar{A}_{\mu}^{a}+\delta A_{\mu}^{a}$, where the fluctuation is taken to be a plane wave,

$$
\delta A_{\mu}^{a}=u_{2}^{a} \epsilon_{2 \mu} e^{i k \cdot x}
$$

choosing an arbitrary color vector $u_{2}$ and spacelike polarization $\epsilon_{2}$. In vacuum, where $\bar{A}_{\mu}^{a}=0$, eq. (4.3) is a solution of the equations of motion (4.1) provided $k^{2}=k \cdot \epsilon_{2}=0$. However, for general backgrounds, this does not solve the unperturbed equations of motion, due to the self-interaction of the gluon. If we take the background $\bar{A}_{\mu}^{a}$ to be given by the constant solution in eq. (4.2), then we require $u_{1,2}$ to satisfy eq. (3.13) in order for the null plane wave perturbation to solve its equation of motion (i.e., to get $k^{2}=0$ as a solution of the dispersion relation at zeroth order in the $c_{i}$ ). In spite of eq. (3.13), there are additional color combinations we can form using $d^{a b c}$, which we define as $U^{a}=d^{a b c} u_{1}^{b} u_{2}^{c}$, $V^{a}=d^{a b c} u_{1}^{b} u_{1}^{c}$, and $W^{a}=d^{a b c} u_{2}^{b} u_{2}^{c}$. The identity in eq. (3.6) relates these as

$$
V W=U^{2}+\frac{2}{N}\left[\left(u_{1} u_{2}\right)^{2}-u_{1}^{2} u_{2}^{2}\right]
$$

where we contract color indices using $\delta^{a b}$.

Within this framework, we now compute the speed $v$ of propagation of our fluctuation to leading order in the $c_{i}$, as in section 2 writing $k_{\mu}=\left(k_{0}, \mathbf{k}\right)$ and identifying the speed $v$ as $k_{0} /|\mathbf{k}|$. To our given order in perturbation theory, we find

$$
\begin{array}{r}
v=1-\frac{8}{M^{4} \epsilon_{2}^{2} u_{2}^{2} k_{0}^{2} N}\left\{\left[\left(\epsilon_{1} \cdot k\right)\left(\epsilon_{2} \cdot \ell\right)-(k \cdot \ell)\left(\epsilon_{1} \cdot \epsilon_{2}\right)\right]^{2} A+\left(\epsilon_{1}^{\mu} \epsilon_{2}^{\nu} k^{\rho} \ell^{\sigma} \epsilon_{\mu \nu \rho \sigma}\right)^{2} B\right. \\
\left.-\left[\left(\epsilon_{1} \cdot k\right)\left(\epsilon_{2} \cdot \ell\right)-(k \cdot \ell)\left(\epsilon_{1} \cdot \epsilon_{2}\right)\right] \epsilon_{1}^{\mu} \epsilon_{2}^{\nu} k^{\rho} \ell^{\sigma} \epsilon_{\mu \nu \rho \sigma} C\right\},
\end{array}
$$

where $A, B$, and $C$ are combinations of the operator coefficients defined as

$$
\begin{aligned}
& A=N\left[\left(2 c_{1}+c_{3}\right)\left(u_{1} u_{2}\right)^{2}+c_{3} u_{1}^{2} u_{2}^{2}+2\left(c_{5}+c_{7}\right) U^{2}\right]+2 c_{7}\left[\left(u_{1} u_{2}\right)^{2}-u_{1}^{2} u_{2}^{2}\right] \\
& B=N\left[\left(2 c_{2}+c_{4}\right)\left(u_{1} u_{2}\right)^{2}+c_{4} u_{1}^{2} u_{2}^{2}+2\left(c_{6}+c_{8}\right) U^{2}\right]+2 c_{8}\left[\left(u_{1} u_{2}\right)^{2}-u_{1}^{2} u_{2}^{2}\right] \\
& C=N\left[\left(2 \widetilde{c}_{1}+\widetilde{c}_{2}\right)\left(u_{1} u_{2}\right)^{2}+\widetilde{c}_{2} u_{1}^{2} u_{2}^{2}+2\left(\widetilde{c}_{3}+\widetilde{c}_{4}\right) U^{2}\right]+2 \widetilde{c}_{4}\left[\left(u_{1} u_{2}\right)^{2}-u_{1}^{2} u_{2}^{2}\right] .
\end{aligned}
$$

Normalizing the color vectors such that $u_{1}^{2}=u_{2}^{2}=1$ will allow us to define $u_{1} u_{2}=\cos \zeta$. For concreteness, we can use Lorentz invariance to fix the direction of $\mathbf{k}$ and the direction of the (spatial vector) $\epsilon_{2}^{\mu}$ orthogonal to $k$. Let us take $\mathbf{k}$ to point in the $z$-direction and $\epsilon_{2}^{\mu}$ to be a unit vector pointing in the $x$-direction. Then let us define parameters $\theta_{1,2,3}, \phi_{1,2,3}$, and $E$ via

$$
\begin{aligned}
\ell_{\mu} & =\left(\cos \theta_{1}, \sin \theta_{1} \cos \theta_{2}, \sin \theta_{1} \sin \theta_{2} \cos \theta_{3}, \sin \theta_{1} \sin \theta_{2} \sin \theta_{3}\right) \\
\epsilon_{1}^{\mu} & =E\left(\cos \phi_{1}, \sin \phi_{1} \cos \phi_{2}, \sin \phi_{1} \sin \phi_{2} \cos \phi_{3}, \sin \phi_{1} \sin \phi_{2} \sin \phi_{3}\right) .
\end{aligned}
$$

\footnotetext{
${ }^{12}$ For the dispersion relation calculation, we work in the geometric optics limit, so that we can approximate any solution with constant $F_{\mu \nu}^{a}$ provided that we ultimately consider perturbations on length scales small compared to the characteristic scale of the background.
} 
Note that we do not need to specify a separate overall scale for both $\ell$ and $\epsilon_{1}$; since each term in the speed correction in eq. (4.5) is quadratic in each of these vectors individually, we can absorb the normalization into the constant $E$ in eq. (4.7). Then the speed $v$ is

$$
v=1-\frac{8 E^{2}}{M^{4} N}\left(A X^{2}+B Y^{2}+C X Y\right),
$$

where

$$
\left(\begin{array}{l}
X \\
Y
\end{array}\right)=\left(\begin{array}{cc}
\sin \theta_{1} \cos \theta_{2} & \sin \phi_{1} \cos \phi_{2} \\
\sin \theta_{1} \sin \theta_{2} \cos \theta_{3} & \sin \phi_{1} \sin \phi_{2} \cos \phi_{3}
\end{array}\right)\left(\begin{array}{c}
\cos \phi_{1}+\sin \phi_{1} \sin \phi_{2} \sin \phi_{3} \\
\cos \theta_{1}-\sin \theta_{1} \sin \theta_{2} \sin \theta_{3}
\end{array}\right) .
$$

Writing $X=Z \cos \psi$ and $Y=Z \sin \psi$ for some $Z>0$, the causality bound becomes

$$
A \cos ^{2} \psi+B \sin ^{2} \psi+C \cos \psi \sin \psi>0 .
$$

We can alternately obtain the constraint in eq. (4.10) from analyticity and unitarity of scattering amplitudes. To do so, we compute the two-to-two gluon scattering amplitude for gluons ${ }^{13}$ with incoming momenta $k_{1,2,3,4}$, polarizations $\epsilon_{1,2,3,4}$, and color vectors $u_{1,2,3,4}$. Let us choose forward kinematics $\left(k_{3}=-k_{1}, k_{4}=-k_{2}, \epsilon_{3}=\epsilon_{1}, \epsilon_{4}=\epsilon_{2}, u_{3}=u_{1}, u_{4}=u_{2}\right)$ and require that $u_{i}$ and $\epsilon_{i}$ be real vectors. As discussed in section 3.2, by requiring that $u_{1,2}$ be chosen such that $f^{a b c} u_{1}^{a} u_{2}^{b}=0$ as in the causality bound, we ensure that contributions from the Yang-Mills cubic interaction and any dimension-six cubics vanish. The forward amplitude is then

$$
\begin{aligned}
\mathcal{A}_{F^{4}}(s) & =\frac{8}{M^{4} N}\left\{A\left(\epsilon_{1} \cdot \epsilon_{2}\right)^{2} s^{2}+B\left[\epsilon_{1}^{2} \epsilon_{2}^{2}-\left(\epsilon_{1} \cdot \epsilon_{2}\right)^{2}\right] s^{2}+2 C\left(\epsilon_{1} \cdot \epsilon_{2}\right) \epsilon_{1}^{\mu} \epsilon_{2}^{\nu} k_{1}^{\rho} k_{2}^{\sigma} \epsilon_{\mu \nu \rho \sigma} s\right\} \\
& =\frac{8 s^{2}}{M^{4} N}\left[A \cos ^{2} \psi+B \sin ^{2} \psi+C \cos \psi \sin \psi\right],
\end{aligned}
$$

where in the second line $\psi$ is now the angle between the unit vectors $\epsilon_{1}$ and $\epsilon_{2}$, defined with sign such that the cross product of $\epsilon_{1}$ and $\epsilon_{2}$ points along the spatial direction of $k_{1}$. Thus, analyticity of gluon-gluon scattering requires the same bound in eq. (4.10) as implied by causality. Marginalizing over $\psi$, we have the requirements: ${ }^{14}$

$$
A>0, \quad B>0, \quad \text { and } \quad C^{2}<4 A B,
$$

which must be satisfied for all $u_{1,2}$ for which $f^{a b c} u_{1}^{a} u_{2}^{b}=0$. Note that if all the CPconserving terms vanish (so that $A=B=0$ ), then the CP-violating terms are forced to vanish as well, since all our Wilson coefficients are real; we will discuss this feature further in section 5.3.

\footnotetext{
${ }^{13}$ Even though gluons in realistic scattering experiments would be confined into nonperturbative objects like hadrons, it is still well-defined to consider the perturbative S-matrix for asymptotic states of gluons and analyze its analytic structure. Indeed, the properties of gluon amplitudes are among the central objects of study in the modern amplitudes program [96-101]. Our analyticity constraints therefore derive from considering such an idealized thought experiment for gluon scattering.

${ }^{14}$ In particular, one obtains $A>0$ by setting $\psi=0, B>0$ by setting $\psi=\pi / 2$, and $C^{2}<4 A B$ by setting $\psi= \pm \arctan \sqrt{A / B}$. One does not find the third bound on the CP-violating term by considering strictly parallel or perpendicular polarizations alone; equivalently, it would not be obtained by considering positivity bounds from fixed-helicity amplitudes, but only from superpositions thereof.
} 


\subsection{1 $\mathrm{SU}(3)$}

Let us determine the consequences of the bound in eq. (4.12) for $\mathrm{SU}(3)_{C}$. For $N=3$, we can rewrite $\mathcal{O}_{7}, \mathcal{O}_{8}$, and $\widetilde{\mathcal{O}}_{4}$ in table 4 in terms of the other operators, resulting in the basis for $\mathcal{O}_{i}^{G^{4}}, \widetilde{\mathcal{O}}_{i}^{G^{4}}$ in table 1. Moreover, for $\mathrm{SU}(3)$ the identities in eqs. (3.6) and (3.9) imply that $U^{2}=u_{1}^{2} u_{2}^{2} / 3$, so we can redefine $A, B, C$ as

$$
\begin{aligned}
& A=3 c_{3}^{G^{4}}+2 c_{5}^{G^{4}}+3\left(2 c_{1}^{G^{4}}+c_{3}^{G^{4}}\right) \cos ^{2} \zeta \\
& \left.B=3 c_{4}^{G^{4}}+2 c_{6}^{G^{4}}+3\left(2 c_{2}^{G^{4}}+c_{4}^{G^{4}}\right) \cos ^{2} \zeta \quad \text { [for } \mathrm{SU}(3)\right] \\
& C=3 \widetilde{c}_{2}^{G^{4}}+2 \widetilde{c}_{3}^{G^{4}}+3\left(2 \widetilde{c}_{1}^{G^{4}}+\widetilde{c}_{2}^{G^{4}}\right) \cos ^{2} \zeta .
\end{aligned}
$$

Since the bound in eq. (4.12) is now linear in $\cos ^{2} \zeta$, we obtain a basis of bounds by considering the two cases of $\cos ^{2} \zeta=0$ or 1 , so the independent bounds are

$$
\begin{aligned}
3 c_{1}^{G^{4}}+3 c_{3}^{G^{4}}+c_{5}^{G^{4}} & >0 \\
3 c_{3}^{G^{4}}+2 c_{5}^{G^{4}} & >0 \\
3 c_{2}^{G^{4}}+3 c_{4}^{G^{4}}+c_{6}^{G^{4}} & >0 \\
3 c_{4}^{G^{4}}+2 c_{6}^{G^{4}} & >0 \\
\left(3 \widetilde{c}_{1}^{G^{4}}+3 \widetilde{c}_{2}^{G^{4}}+\widetilde{c}_{3}^{G^{4}}\right)^{2} & <4\left(3 c_{1}^{G^{4}}+3 c_{3}^{G^{4}}+c_{5}^{G^{4}}\right)\left(3 c_{2}^{G^{4}}+3 c_{4}^{G^{4}}+c_{6}^{G^{4}}\right) \\
\left(3 \widetilde{c}_{2}^{G^{4}}+2 \widetilde{c}_{3}^{G^{4}}\right)^{2} & <4\left(3 c_{3}^{G^{4}}+2 c_{5}^{G^{4}}\right)\left(3 c_{4}^{G^{4}}+2 c_{6}^{G^{4}}\right) .
\end{aligned}
$$

\subsection{2 $\mathrm{SU}(2)$}

For the case of $\mathrm{SU}(2)_{L}$, the basis and bounds simplify further. The $d^{a b c}$ coefficients all vanish for $\mathrm{SU}(2)$, so we reduce to the basis of $\mathcal{O}_{i}^{W^{4}}, \widetilde{\mathcal{O}}_{i}^{W^{4}}$ in table 1 and have $U^{2}=0$. Moreover, since the structure constants for SU(2) are simply $\epsilon^{I J K}$, the requirement in eq. (3.13) implies that we must have $u_{1}= \pm u_{2}$. Thus, we can now redefine $A, B, C$ for $\mathrm{SU}(2)$ as

$$
\begin{aligned}
& A=4\left(c_{1}^{W^{4}}+c_{3}^{W^{4}}\right) \\
& B=4\left(c_{2}^{W^{4}}+c_{4}^{W^{4}}\right) \quad[\text { for } \mathrm{SU}(2)] \\
& C=4\left(\widetilde{c}_{1}^{W^{4}}+\widetilde{c}_{2}^{W^{4}}\right),
\end{aligned}
$$

so the independent bounds become

$$
\begin{array}{rlr}
c_{1}^{W^{4}}+c_{3}^{W^{4}} & >0 \\
c_{2}^{W^{4}}+c_{4}^{W^{4}} & >0 \\
\left(\widetilde{c}_{1}^{W^{4}}+\widetilde{c}_{2}^{W^{4}}\right)^{2} & <4\left(c_{1}^{W^{4}}+c_{3}^{W^{4}}\right)\left(c_{2}^{W^{4}}+c_{4}^{W^{4}}\right) . &
\end{array}
$$

\subsubsection{U(1)}

For the $\mathrm{U}(1)_{Y}$ case, all the color structure disappears, and we are left with only the three $B^{4}$ operators in table 1 . We can write

$$
\begin{aligned}
& A=2 c_{1}^{B^{4}} \\
& \left.B=2 c_{2}^{B^{4}} \quad \text { [for } \mathrm{U}(1)\right] \\
& C=2 \widetilde{c}_{1}^{B^{4}},
\end{aligned}
$$


so the independent bounds become

$$
\begin{aligned}
c_{1}^{B^{4}} & >0 \\
c_{2}^{B^{4}} & >0 \\
\left(\widetilde{c}_{1}^{B^{4}}\right)^{2} & <4 c_{1}^{B^{4}} c_{2}^{B^{4}} .
\end{aligned}
$$

The first two lines of eq. (4.18) match the results derived in ref. [34], and the third line can further be obtained by demanding index of refraction greater than unity in the vacuum birefringence calculation of ref. [102]; see also refs. [103, 104]. Discussion of experimental limits on the photonic component of $c_{i}^{B^{4}}$ can be found in ref. [105].

That the SU(2) and SU(3) results in eq. (4.16) and eq. (4.14) take the form of single and pairwise copies of the $\mathrm{U}(1)$ bounds in eq. (4.18) is not by accident. As commented above, it is due to our insistence on imposing eq. (3.13), which restricts us to the Cartan subalgebra in each case. For $\mathrm{SU}(N)$ the Cartan subalgebra consists of $N-1$ copies of $\mathrm{U}(1)$, explaining this pattern between the bounds.

\subsection{Field strength cross-quartics}

Let us next bound the cross-quartic operators among the gauge field strengths appearing in table 1. For a Yang-Mills theory with gauge group $\mathrm{SU}(N) \otimes \mathrm{SU}(n)$ for $N, n$ arbitrary with respective gauge fields $A_{\mu}^{a}$ and $a_{\mu}^{A}$ and field strengths $F_{\mu \nu}^{a}$ and $f_{\mu \nu}^{A}$, let us again choose a background for $A_{\mu}^{a}$ as in eq. (4.2). The ansatz for the perturbation of the $\mathrm{SU}(n)$ gauge field, $\delta a_{\mu}^{A}$, will be written as in eq. (4.3), $u_{2}^{A} \epsilon_{2 \mu} e^{i k \cdot x}$. We define the coordinate system for $\epsilon_{1,2}$ and $\partial_{\mu} w$ as in section 4.1 .

Writing the basis of cross-quartic operators analogously with those in table 1 as

$$
\begin{aligned}
& \mathcal{O}_{1}=F_{\mu \nu}^{a} F^{a \mu \nu} f_{\rho \sigma}^{A} f^{A \rho \sigma} \\
& \mathcal{O}_{2}=F_{\mu \nu}^{a} \widetilde{F}^{a \mu \nu} f_{\rho \sigma}^{A} \widetilde{f}^{A \rho \sigma} \\
& \mathcal{O}_{3}=F_{\mu \nu}^{a} f^{A \mu \nu} F_{\rho \sigma}^{a} f^{A \rho \sigma} \\
& \mathcal{O}_{4}=F_{\mu \nu}^{a} \widetilde{f}^{A \mu \nu} \widetilde{F}_{\rho \sigma}^{a} f^{A \rho \sigma} \\
& \widetilde{\mathcal{O}}_{1}=F_{\mu \nu}^{a} \widetilde{F}^{a \mu \nu} f_{\rho \sigma}^{A} f^{A \rho \sigma} \\
& \widetilde{\mathcal{O}}_{2}=F_{\mu \nu}^{a} F^{a \mu \nu} f_{\rho \sigma}^{A} \widetilde{f}^{A \rho \sigma} \\
& \widetilde{\mathcal{O}}_{3}=F_{\mu \nu}^{a} f^{A \mu \nu} \widetilde{F}_{\rho \sigma}^{a} f^{A \rho \sigma},
\end{aligned}
$$

with Wilson coefficients $c_{i} / M^{4}, \widetilde{c}_{i} / M^{4}$, we find that the speed of propagation of the $\delta a_{\mu}^{A}$ fluctuations is

$$
v=1-\frac{4 E^{2}}{M^{4}}\left(c_{3} X^{2}+c_{4} Y^{2}+\widetilde{c}_{3} X Y\right)
$$

for $X, Y$ defined in eq. (4.9). Similarly, the four-point amplitude becomes

$$
\mathcal{A}_{F^{2} f^{2}}(s)=\frac{4 s^{2}}{M^{4}}\left[c_{3} \cos ^{2} \psi+c_{4} \sin ^{2} \psi+\widetilde{c}_{3} \cos \psi \sin \psi\right],
$$

so the bounds from analyticity and causality are identical and, marginalizing over $\psi$, can be written simply as the requirements that $c_{3}>0, c_{4}>0$, and $\widetilde{c}_{3}^{2}<4 c_{3} c_{4}$. 
Applying this result to the operators in table 1, we obtain the bounds on the SMEFT:

$$
\begin{aligned}
c_{3}^{B^{2} W^{2}} & >0 \\
c_{4}^{B^{2} W^{2}} & >0 \\
\left(\widetilde{c}_{3}^{B^{2} W^{2}}\right)^{2} & <4 c_{3}^{B^{2} W^{2}} c_{4}^{B^{2} W^{2}} \\
c_{3}^{B^{2} G^{2}} & >0 \\
c_{4}^{B^{2} G^{2}} & >0 \\
\left(\widetilde{c}_{3}^{B^{2} G^{2}}\right)^{2} & <4 c_{3}^{B^{2} G^{2}} c_{4}^{B^{2} G^{2}} \\
c_{3}^{W^{2} G^{2}} & >0 \\
c_{4}^{W^{2} G^{2}} & >0 \\
\left(\widetilde{c}_{3}^{W^{2} G^{2}}\right)^{2} & <4 c_{3}^{W^{2} G^{2}} c_{4}^{W^{2} G^{2}} .
\end{aligned}
$$

\subsection{Higgs quartics}

Let us now bound the dimension-eight operators quartic in the Higgs in table 2. We will be considering the change to the speed of Higgs propagation in nonzero Higgs backgrounds, as well as Higgs four-point scattering. As we reviewed in section 2, a positivity bound on the coefficient of $(\partial \phi)^{4}$ was proven for a real massless scalar $\phi$ in ref. [34]; for the $\mathcal{O}_{i}^{H^{4}}$, however, the $\mathrm{SU}(2)$ doublet nature of the SM Higgs field leads to more interesting structure in the bounds. Since we are considering a completion scale $M$ above the weak scale, we can approximate the Higgs as effectively massless in our dispersion relation calculation.

Expanding the covariant derivatives and considering backgrounds with zero gauge field, the $\mathcal{O}_{i}^{H^{4}}$ operators become $(\partial H)^{4}$. Expanding in the $\phi_{1,2,3,4}$ defined in eq. (3.10), we have

$$
\begin{aligned}
\mathcal{O}_{1}^{H^{4}} \rightarrow & \frac{1}{4} \sum_{i=1}^{4}\left(\partial \phi_{i}\right)^{4}+\frac{1}{2}\left[\left(\partial_{\mu} \phi_{1} \partial^{\mu} \phi_{3}\right)^{2}+\left(\partial_{\mu} \phi_{1} \partial^{\mu} \phi_{4}\right)^{2}+\left(\partial_{\mu} \phi_{2} \partial^{\mu} \phi_{3}\right)^{2}+\left(\partial_{\mu} \phi_{2} \partial^{\mu} \phi_{4}\right)^{2}\right] \\
& +\frac{1}{2}\left[\left(\partial \phi_{1}\right)^{2}\left(\partial \phi_{2}\right)^{2}+\left(\partial \phi_{3}\right)^{2}\left(\partial \phi_{4}\right)^{2}\right]-\partial_{\mu} \phi_{1} \partial^{\mu} \phi_{4} \partial_{\nu} \phi_{2} \partial^{\nu} \phi_{3}+\partial_{\mu} \phi_{1} \partial^{\mu} \phi_{3} \partial_{\nu} \phi_{2} \partial^{\nu} \phi_{4} \\
\mathcal{O}_{2}^{H^{4}} \rightarrow & \frac{1}{4} \sum_{i=1}^{4}\left(\partial \phi_{i}\right)^{4}+\frac{1}{2}\left[\left(\partial_{\mu} \phi_{1} \partial^{\mu} \phi_{3}\right)^{2}+\left(\partial_{\mu} \phi_{1} \partial^{\mu} \phi_{4}\right)^{2}+\left(\partial_{\mu} \phi_{2} \partial^{\mu} \phi_{3}\right)^{2}+\left(\partial_{\mu} \phi_{2} \partial^{\mu} \phi_{4}\right)^{2}\right] \\
& -\frac{1}{2}\left[\left(\partial \phi_{1}\right)^{2}\left(\partial \phi_{2}\right)^{2}+\left(\partial \phi_{3}\right)^{2}\left(\partial \phi_{4}\right)^{2}\right]+\left(\partial_{\mu} \phi_{1} \partial^{\mu} \phi_{2}\right)^{2}+\left(\partial_{\mu} \phi_{3} \partial^{\mu} \phi_{4}\right)^{2} \\
& +\partial_{\mu} \phi_{1} \partial^{\mu} \phi_{4} \partial_{\nu} \phi_{2} \partial^{\nu} \phi_{3}-\partial_{\mu} \phi_{1} \partial^{\mu} \phi_{3} \partial_{\nu} \phi_{2} \partial^{\nu} \phi_{4} \\
\mathcal{O}_{3}^{H^{4}} \rightarrow & \frac{1}{4} \sum_{i=1}^{4}\left(\partial \phi_{i}\right)^{4}+\frac{1}{2}\left[\left(\partial \phi_{1}\right)^{2}\left(\partial \phi_{2}\right)^{2}+\left(\partial \phi_{1}\right)^{2}\left(\partial \phi_{3}\right)^{2}+\left(\partial \phi_{2}\right)^{2}\left(\partial \phi_{3}\right)^{2}\right] \\
& +\frac{1}{2}\left[\left(\partial \phi_{1}\right)^{2}\left(\partial \phi_{4}\right)^{2}+\left(\partial \phi_{2}\right)^{2}\left(\partial \phi_{4}\right)^{2}+\left(\partial \phi_{3}\right)^{2}\left(\partial \phi_{4}\right)^{2}\right] .
\end{aligned}
$$

Let us now compute the effect of the operators in eq. (4.23) on the speed of Higgs propagation. In a background of nonzero $\bar{\phi}_{i}$ for $i \in\{1,2,3,4\}$, for perturbations $\delta \phi_{i} \propto e^{i k \cdot x}$ with wavelengths much smaller than the characteristic scale of the background, $\bar{\phi}_{i}$ can be expanded as a constant vacuum expectation value plus a constant derivative $\overline{\partial_{\mu} \phi_{i}}=j_{\mu}$. 
We choose this background to be smaller than the weak scale, so that it does not induce an effective Higgs mass via the Higgs quartic or higher-dimension operators. We then find a speed of fluctuations equal to

$$
v=1-\left(c_{1}^{H^{4}}+c_{2}^{H^{4}}+c_{3}^{H^{4}}\right) \frac{(j \cdot k)^{2}}{k_{0}^{2} M^{4}},
$$

so we must have $c_{1}^{H^{4}}+c_{2}^{H^{4}}+c_{3}^{H^{4}}>0$.

Similarly, if we take a background of $\phi_{1}$ or $\phi_{2}$, and consider perturbations in $\phi_{3}$ or $\phi_{4}$ (or vice versa), we have

$$
v=1-\left(c_{1}^{H^{4}}+c_{2}^{H^{4}}\right) \frac{(j \cdot k)^{2}}{2 k_{0}^{2} M^{4}},
$$

so we must have $c_{1}^{H^{4}}+c_{2}^{H^{4}}>0$. Finally, if we take a background of $\phi_{1}$ (or $\phi_{3}$ ) and consider perturbations in $\phi_{2}$ (or $\phi_{4}$, respectively), or vice versa, we obtain

$$
v=1-c_{2}^{H^{4}} \frac{(j \cdot k)^{2}}{k_{0}^{2} M^{4}}
$$

so we must have $c_{2}^{H^{4}}>0$.

We can arrive at these same bounds from analyticity of two-to-two Higgs scattering. Let us compute an amplitude for particles I and II scattering to particles III and IV. ${ }^{15}$ For each of particles I, II, III, IV, let us consider an arbitrary superposition of $\phi_{i}$ states:

$$
\begin{array}{rlrl}
|\mathrm{I}\rangle & =\sum_{i=1}^{4} \alpha_{i}\left|\phi_{i}\right\rangle & |\mathrm{III}\rangle & =\sum_{i=1}^{4} \gamma_{i}\left|\phi_{i}\right\rangle \\
|\mathrm{II}\rangle=\sum_{i=1}^{4} \beta_{i}\left|\phi_{i}\right\rangle & |\mathrm{IV}\rangle=\sum_{i=1}^{4} \delta_{i}\left|\phi_{i}\right\rangle
\end{array}
$$

where $\sum_{i}\left|\alpha_{i}\right|^{2}=\sum_{i}\left|\beta_{i}\right|^{2}=\sum_{i}\left|\gamma_{i}\right|^{2}=\sum_{i}\left|\delta_{i}\right|^{2}=1$. To apply the optical theorem, we must consider forward scattering, which means $k_{\mathrm{I}}=-k_{\mathrm{III}}$ and $k_{\mathrm{II}}=-k_{\mathrm{IV}}$ in the all-incoming convention and $\alpha_{i}=\gamma_{i}^{*}$ and $\beta_{i}=\delta_{i}^{*}$. Schematically, the $s^{2}$ part of the forward amplitude coming from the $\mathcal{O}_{i}^{H^{4}}$ is then

$$
\mathcal{A}_{H^{4}}(s)=\sum_{i j k l} K_{i j k l} \alpha_{i} \beta_{j} \alpha_{k}^{*} \beta_{l}^{*} \frac{s^{2}}{M^{4}},
$$

where $K_{i j k l}$ is a rank-four tensor, with each entry proportional to a sum of $c_{1,2,3}^{H^{4}}$. Analogously with polarizations, to ensure for simplicity that $\mathcal{A}_{H^{4}}(s)$ maps onto itself under crossing, we choose $\alpha_{i}$ and $\beta_{i}$ to be real. Analyticity and unitarity then require

$$
\sum_{i j k l} K_{i j k l} \alpha_{i} \beta_{j} \alpha_{k} \beta_{l}>0
$$

\footnotetext{
${ }^{15}$ We use $|\mathrm{I}\rangle|\mathrm{II}\rangle \rightarrow|\mathrm{III}\rangle|\mathrm{IV}\rangle$ here, instead of our usual $12 \rightarrow 34$ notation, to avoid ambiguity with the four Higgs fields, which we have already labeled 1234 .
} 
for all $\alpha_{i}, \beta_{i}$. Numerical analysis shows that the analyticity and unitarity conditions in eq. (4.29) can be reduced to

$$
\begin{aligned}
c_{1}^{H^{4}}+c_{2}^{H^{4}}+c_{3}^{H^{4}} & >0 \\
c_{1}^{H^{4}}+c_{2}^{H^{4}} & >0 \\
c_{2}^{H^{4}} & >0
\end{aligned}
$$

and that these conditions are unchanged even if we let $\alpha_{i}$ and $\beta_{i}$ be complex (i.e., marginalize over $K_{i j k l} \alpha_{i} \beta_{j} \alpha_{k}^{*} \beta_{l}^{*}$ ). These are precisely the same conditions we obtained from causality. The first bound in eq. (4.30) can be obtained from scattering $\phi_{i} \phi_{i} \rightarrow \phi_{i} \phi_{i}$ (all the same state) for any $i \in\{1,2,3,4\}$, in which case $\mathcal{A}_{H^{4}}=\left(c_{1}^{H^{4}}+c_{2}^{H^{4}}+c_{3}^{H^{4}}\right) s^{2} / M^{4}$. The second bound can be obtained from scattering $\phi_{1} \phi_{3} \rightarrow \phi_{1} \phi_{3}, \phi_{1} \phi_{4} \rightarrow \phi_{1} \phi_{4}, \phi_{2} \phi_{3} \rightarrow \phi_{2} \phi_{3}$, or $\phi_{2} \phi_{4} \rightarrow \phi_{2} \phi_{4}$, in which case $\mathcal{A}_{H^{4}}=\left(c_{1}^{H^{4}}+c_{2}^{H^{4}}\right) s^{2} / 2 M^{4}$. Finally, the third bound can be obtained from scattering $\phi_{1} \phi_{2} \rightarrow \phi_{1} \phi_{2}$ or $\phi_{3} \phi_{4} \rightarrow \phi_{3} \phi_{4}$, in which case $\mathcal{A}_{H^{4}}=c_{2}^{H^{4}} s^{2} / M^{4}$.

\subsection{Higgs/field strength cross-quartics}

Let us now bound the coefficients of operators in table 2 that give cross-quartic couplings between the Higgs and gauge bosons. Since we will be considering the speed of fluctuations and scattering amplitudes for bosons of definite type (i.e., $H, B, W$, or $G$ ), we will be bounding operators of the type $\mathcal{O}_{i}^{H^{2} F^{2}}$, for $F=B, W, G$.

Within a Higgs background $\overline{\partial_{\mu} \phi_{i}}$ as described in section 4.3 , a $B, W$, or $G$ boson's speed of propagation is corrected by the operators in table 2 to

$$
\begin{aligned}
v & =1-c_{1}^{H^{2} B^{2}} \frac{(j \cdot k)^{2}}{2 k_{0}^{2} M^{4}} \\
v & =1-c_{1}^{H^{2} W^{2}} \frac{(j \cdot k)^{2}}{2 k_{0}^{2} M^{4}} \\
\text { or } v & =1-c_{1}^{H^{2} G^{2}} \frac{(j \cdot k)^{2}}{2 k_{0}^{2} M^{4}},
\end{aligned}
$$

respectively. If we instead have a gauge field background of $B, W$, or $G$ as defined in section 4.1 and compute the speed of propagation for a perturbation of the Higgs field, we have

$$
\begin{gathered}
v=1-\frac{E^{2}}{2 M^{4}} c_{1}^{H^{2} B^{2}}\left(X^{2}+Y^{2}\right) \\
v=1-\frac{E^{2}}{2 M^{4}} c_{1}^{H^{2} W^{2}}\left(X^{2}+Y^{2}\right) \\
\text { or } v=1-\frac{E^{2}}{2 M^{4}} c_{1}^{H^{2} G^{2}}\left(X^{2}+Y^{2}\right),
\end{gathered}
$$

respectively. Thus, causality requires ${ }^{16}$

$$
\begin{aligned}
c_{1}^{H^{2} B^{2}} & >0 \\
c_{1}^{H^{2} W^{2}} & >0 \\
c_{1}^{H^{2} G^{2}} & >0 .
\end{aligned}
$$

\footnotetext{
${ }^{16}$ We note that in the mostly-minus metric convention, the opposite of what we use in this work, these bounds all become $c_{1}^{H^{2} F^{2}}<0$. This sign change in the bound arises as the associated operators involve an odd number of Lorentz contractions and therefore also change signs between signatures. These are the only bounds presented in this work that vary with signature convention.
} 
We can check that analyticity of scattering amplitudes gives the same bounds. Scattering $H B \rightarrow H B$ in the forward limit, we have, at $\mathcal{O}\left(s^{2}\right)$,

$$
\mathcal{A}(s)_{H^{2} B^{2}}=c_{1}^{H^{2} B^{2}} \frac{s^{2}}{2 M^{4}} .
$$

As in section 4.3, we can ignore any SM contribution to this process, since the contour integral discussed in section 2 will extract the $s^{2}$ term in the forward amplitude. Similarly for forward $H W \rightarrow H W$ and $H G \rightarrow H G$ scattering, we obtain, at $\mathcal{O}\left(s^{2}\right)$,

$$
\begin{aligned}
& \mathcal{A}(s)_{H^{2} W^{2}}=c_{1}^{H^{2} W^{2}} \frac{s^{2}}{2 M^{4}} \\
& \mathcal{A}(s)_{H^{2} G^{2}}=c_{1}^{H^{2} G^{2}} \frac{s^{2}}{2 M^{4}} .
\end{aligned}
$$

Thus, analyticity implies the same bounds as causality for Higgs/field strength crossquartics, given in eq. (4.33).

\section{UV completions}

It is instructive to check whether the EFT coefficients in example theories satisfy the positivity bounds we derived in section 4. We should expect the EFT coefficients arising from any well defined field theoretic completion to automatically obey these bounds. By verifying that the inequalities are manifestly satisfied for a large class of completions, we thus perform a nontrivial consistency check of our calculation.

In all of the examples considered in this section, we will analyze the operator coefficients generated at the scale where the new physics is integrated out. This qualifier is important: at lower energy scales, the coefficients will generically run and mix under renormalizationgroup evolution. While the form of our bounds is independent of these details, if one wanted to convert the operator coefficients appearing in our bounds at a given scale into either the parameters of the UV theory, or the parameters of the EFT at the matching scale, these complications must be accounted for. For the analysis in this section, we could require that the UV theory is sufficiently weakly coupled that, at the scale we imagine performing our scattering experiments, the matching coefficients remain the only relevant contributions to the dimension- 8 operators.

\subsection{One-loop completions of gauge field operators}

Let us consider introducing some extra massive field $\Phi$, with mass $M$, charged under the SM gauge group. ${ }^{17}$ We will let $\Phi$ be a complex scalar, Dirac fermion, or complex vector field, in an arbitrary irreducible representation of $\mathrm{SU}(3)_{C} \otimes \mathrm{SU}(2)_{L} \otimes \mathrm{U}(1)_{Y}$. Let us write the representation under $\mathrm{SU}(3)_{C}$ as $\mathbf{R}_{3}$, the representation under $\mathrm{SU}(2)_{L}$ as $\mathbf{R}_{2}$, and the charge under $\mathrm{U}(1)_{Y}$ as $Q$.

\footnotetext{
${ }^{17}$ For example, the well known Euler-Heisenberg coefficients for $\mathrm{U}(1)$ electrodynamics, $c_{1,2}^{B^{4}}$, are both positive when the electron is integrated out [106-108], satisfying the positivity bounds of ref. [34]; we wish to substantially generalize this check.
} 


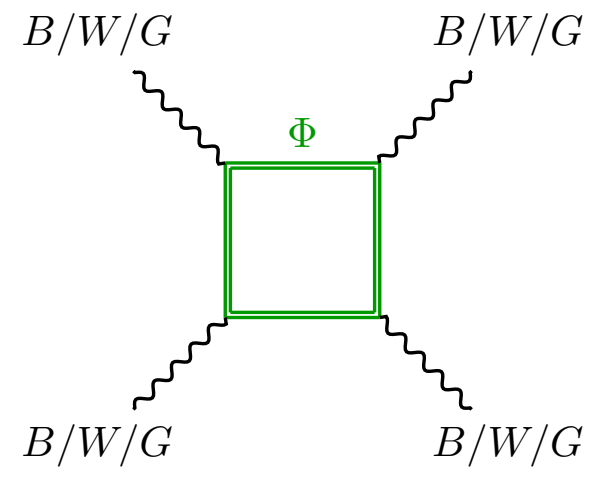

Figure 4. One-loop diagram involving a heavy state $\Phi$, which when integrated out will generate a set of CP-even $F^{4}$ operators, the coefficients of which must obey our bounds. Here $\Phi$ can be a complex scalar, Dirac fermion, or complex vector field.

Integrating out $\Phi$ at one loop generates quartic terms among the gauge field strengths of the form given in table 1, specifically, the CP-conserving operators. The corresponding Feynman diagram is shown in figure 4, where in the EFT below $M$, the loop is replaced by an effective four-point vertex, giving rise to the higher-dimension operators we consider. In ref. [109], the Wilson coefficients for these generalized Euler-Heisenberg terms in the EFT were computed. To translate from the basis of operators in ref. [109] to that in table 1, the following identities are useful: for SU(3),

$$
\begin{aligned}
f^{a b e} f^{c d e} G_{\mu \nu}^{a} G^{c \mu \nu} G_{\rho \sigma}^{b} G^{d \rho \sigma} & =\frac{1}{2} \mathcal{O}_{1}^{G^{4}}-\mathcal{O}_{3}^{G^{4}}+\frac{3}{2} \mathcal{O}_{5}^{G^{4}} \\
f^{a b e} f^{c d e} G_{\mu \nu}^{a} \widetilde{G}^{c \mu \nu} G_{\rho \sigma}^{b} \widetilde{G}^{d \rho \sigma} & =\frac{1}{2} \mathcal{O}_{2}^{G^{4}}-\mathcal{O}_{4}^{G^{4}}+\frac{3}{2} \mathcal{O}_{6}^{G^{4}},
\end{aligned}
$$

and for $\mathrm{SU}(2)$,

$$
\begin{aligned}
\epsilon^{I J M} \epsilon^{K L M} W_{\mu \nu}^{I} W^{K \mu \nu} W_{\rho \sigma}^{J} W^{L \rho \sigma} & =\mathcal{O}_{1}^{W^{4}}-\mathcal{O}_{3}^{W^{4}} \\
\epsilon^{I J M} \epsilon^{K L M} W_{\mu \nu}^{I} \widetilde{W}^{K \mu \nu} W_{\rho \sigma}^{J} \widetilde{W}^{L \rho \sigma} & =\mathcal{O}_{2}^{W^{4}}-\mathcal{O}_{4}^{W^{4}} .
\end{aligned}
$$

We can specify the irreducible representations of $\mathrm{SU}(2)$ and $\mathrm{SU}(3)$ as $\mathbf{R}_{2}=(r)$ and $\mathbf{R}_{3}=(p, q)$, labeled by their Dynkin indices. ${ }^{18}$ Let us define the quadratic invariants for the representations,

$$
\begin{aligned}
I_{2}(r) & =\frac{1}{12} r(r+1)(r+2) \\
I_{2}(p, q) & =\frac{1}{48}(p+1)(q+1)(p+q+2)[p(p+3)+q(q+3)+p q],
\end{aligned}
$$

the cubic anomaly invariant [111], which vanishes for $\mathrm{SU}(2)$,

$$
I_{3}(p, q)=\frac{1}{120}(p+1)(q+1)(p+q+2)(p-q)(p+2 q+3)(q+2 p+3),
$$

\footnotetext{
${ }^{18}$ The number of states in the $\mathrm{SU}(2)$ multiplet is $r+1$. For the $\mathrm{SU}(3)$ multiplet, there are $(p+1)(q+1)(p+$ $q+2) / 2$ particles in the representation, and one can think of the $(p, q)$ of $\mathrm{SU}(3)$ as the number of quarks and antiquarks, respectively, in the representation, corresponding to a Young tableau with $p$ columns of one box and $q$ columns of two boxes (or, equivalently, to the number of steps across the top and bottom, respectively, of the multiplet diagram) [110].
} 


\begin{tabular}{|c|c|c|c|}
\hline & scalar & fermion & vector \\
\hline$c_{1}^{B^{4}}$ & $\frac{7}{32} g_{1}^{4} Q^{4}$ & $\frac{1}{2} g_{1}^{4} Q^{4}$ & $\frac{261}{32} g_{1}^{4} Q^{4}$ \\
\hline$c_{2}^{B^{4}}$ & $\frac{1}{32} g_{1}^{4} Q^{4}$ & $\frac{7}{8} g_{1}^{4} Q^{4}$ & $\frac{243}{32} g_{1}^{4} Q^{4}$ \\
\hline$c_{1}^{W^{4}}$ & $g_{2}^{4}\left[\frac{7}{32} \Lambda\left(\mathbf{R}_{2}\right)+\frac{1}{48} I_{2}\left(\mathbf{R}_{2}\right)\right]$ & $g_{2}^{4}\left[\frac{1}{2} \Lambda\left(\mathbf{R}_{2}\right)+\frac{1}{48} I_{2}\left(\mathbf{R}_{2}\right)\right]$ & $g_{2}^{4}\left[\frac{261}{32} \Lambda\left(\mathbf{R}_{2}\right)-\frac{3}{16} I_{2}\left(\mathbf{R}_{2}\right)\right]$ \\
\hline$c_{2}^{W^{4}}$ & $g_{2}^{4}\left[\frac{1}{32} \Lambda\left(\mathbf{R}_{2}\right)+\frac{1}{336} I_{2}\left(\mathbf{R}_{2}\right)\right]$ & $g_{2}^{4}\left[\frac{7}{8} \Lambda\left(\mathbf{R}_{2}\right)+\frac{19}{336} I_{2}\left(\mathbf{R}_{2}\right)\right]$ & $g_{2}^{4}\left[\frac{243}{32} \Lambda\left(\mathbf{R}_{2}\right)-\frac{27}{112} I_{2}\left(\mathbf{R}_{2}\right)\right]$ \\
\hline$c_{3}^{W^{4}}$ & $g_{2}^{4}\left[\frac{7}{16} \Lambda\left(\mathbf{R}_{2}\right)-\frac{1}{48} I_{2}\left(\mathbf{R}_{2}\right)\right]$ & $g_{2}^{4}\left[\Lambda\left(\mathbf{R}_{2}\right)-\frac{1}{48} I_{2}\left(\mathbf{R}_{2}\right)\right]$ & $g_{2}^{4}\left[\frac{261}{16} \Lambda\left(\mathbf{R}_{2}\right)+\frac{3}{16} I_{2}\left(\mathbf{R}_{2}\right)\right]$ \\
\hline$c_{4}^{W^{4}}$ & $g_{2}^{4}\left[\frac{1}{16} \Lambda\left(\mathbf{R}_{2}\right)-\frac{1}{336} I_{2}\left(\mathbf{R}_{2}\right)\right]$ & $g_{2}^{4}\left[\frac{7}{4} \Lambda\left(\mathbf{R}_{2}\right)-\frac{19}{336} I_{2}\left(\mathbf{R}_{2}\right)\right]$ & $g_{2}^{4}\left[\frac{243}{16} \Lambda\left(\mathbf{R}_{2}\right)+\frac{27}{112} I_{2}\left(\mathbf{R}_{2}\right)\right]$ \\
\hline$c_{1}^{G^{4}}$ & $g_{3}^{4}\left[\frac{7}{32} \Lambda\left(\mathbf{R}_{3}\right)+\frac{1}{96} I_{2}\left(\mathbf{R}_{3}\right)\right]$ & $g_{3}^{4}\left[\frac{1}{2} \Lambda\left(\mathbf{R}_{3}\right)+\frac{1}{96} I_{2}\left(\mathbf{R}_{3}\right)\right]$ & $g_{3}^{4}\left[\frac{261}{32} \Lambda\left(\mathbf{R}_{3}\right)-\frac{3}{32} I_{2}\left(\mathbf{R}_{3}\right)\right]$ \\
\hline$c_{2}^{G^{4}}$ & $g_{3}^{4}\left[\frac{1}{32} \Lambda\left(\mathbf{R}_{3}\right)+\frac{1}{672} I_{2}\left(\mathbf{R}_{3}\right)\right]$ & $g_{3}^{4}\left[\frac{7}{8} \Lambda\left(\mathbf{R}_{3}\right)+\frac{19}{672} I_{2}\left(\mathbf{R}_{3}\right)\right]$ & $g_{3}^{4}\left[\frac{243}{32} \Lambda\left(\mathbf{R}_{3}\right)-\frac{27}{224} I_{2}\left(\mathbf{R}_{3}\right)\right]$ \\
\hline$c_{3}^{G^{4}}$ & $g_{3}^{4}\left[\frac{7}{16} \Lambda\left(\mathbf{R}_{3}\right)-\frac{1}{48} I_{2}\left(\mathbf{R}_{3}\right)\right]$ & $g_{3}^{4}\left[\Lambda\left(\mathbf{R}_{3}\right)-\frac{1}{48} I_{2}\left(\mathbf{R}_{3}\right)\right]$ & $g_{3}^{4}\left[\frac{261}{16} \Lambda\left(\mathbf{R}_{3}\right)+\frac{3}{16} I_{2}\left(\mathbf{R}_{3}\right)\right]$ \\
\hline$c_{4}^{G^{4}}$ & $g_{3}^{4}\left[\frac{1}{16} \Lambda\left(\mathbf{R}_{3}\right)-\frac{1}{336} I_{2}\left(\mathbf{R}_{3}\right)\right]$ & $g_{3}^{4}\left[\frac{7}{4} \Lambda\left(\mathbf{R}_{3}\right)-\frac{19}{336} I_{2}\left(\mathbf{R}_{3}\right)\right]$ & $g_{3}^{4}\left[\frac{243}{16} \Lambda\left(\mathbf{R}_{3}\right)+\frac{27}{112} I_{2}\left(\mathbf{R}_{3}\right)\right]$ \\
\hline$c_{5}^{G^{4}}$ & $\frac{1}{32} g_{3}^{4} I_{2}\left(\mathbf{R}_{3}\right)$ & $\frac{1}{32} g_{3}^{4} I_{2}\left(\mathbf{R}_{3}\right)$ & $-\frac{9}{32} g_{3}^{4} I_{2}\left(\mathbf{R}_{3}\right)$ \\
\hline$c_{6}^{G^{4}}$ & $\frac{1}{224} g_{3}^{4} I_{2}\left(\mathbf{R}_{3}\right)$ & $\frac{19}{224} g_{3}^{4} I_{2}\left(\mathbf{R}_{3}\right)$ & $-\frac{81}{224} g_{3}^{4} I_{2}\left(\mathbf{R}_{3}\right)$ \\
\hline$c_{1}^{B^{2} W^{2}}$ & $\frac{7}{16} g_{1}^{2} g_{2}^{2} Q^{2} I_{2}\left(\mathbf{R}_{2}\right)$ & $g_{1}^{2} g_{2}^{2} Q^{2} I_{2}\left(\mathbf{R}_{2}\right)$ & $\frac{261}{16} g_{1}^{2} g_{2}^{2} Q^{2} I_{2}\left(\mathbf{R}_{2}\right)$ \\
\hline$c_{2}^{B^{2} W^{2}}$ & $\frac{1}{16} g_{1}^{2} g_{2}^{2} Q^{2} I_{2}\left(\mathbf{R}_{2}\right)$ & $\frac{7}{4} g_{1}^{2} g_{2}^{2} Q^{2} I_{2}\left(\mathbf{R}_{2}\right)$ & $\frac{243}{16} g_{1}^{2} g_{2}^{2} Q^{2} I_{2}\left(\mathbf{R}_{2}\right)$ \\
\hline$c_{3}^{B^{2} W^{2}}$ & $\frac{7}{8} g_{1}^{2} g_{2}^{2} Q^{2} I_{2}\left(\mathbf{R}_{2}\right)$ & $2 g_{1}^{2} g_{2}^{2} Q^{2} I_{2}\left(\mathbf{R}_{2}\right)$ & $\frac{261}{8} g_{1}^{2} g_{2}^{2} Q^{2} I_{2}\left(\mathbf{R}_{2}\right)$ \\
\hline$c_{4}^{B^{2} W^{2}}$ & $\frac{1}{8} g_{1}^{2} g_{2}^{2} Q^{2} I_{2}\left(\mathbf{R}_{2}\right)$ & $\frac{7}{2} g_{1}^{2} g_{2}^{2} Q^{2} I_{2}\left(\mathbf{R}_{2}\right)$ & $\frac{243}{8} g_{1}^{2} g_{2}^{2} Q^{2} I_{2}\left(\mathbf{R}_{2}\right)$ \\
\hline$c_{1}^{B^{2} G^{2}}$ & $\frac{7}{16} g_{1}^{2} g_{3}^{2} Q^{2} I_{2}\left(\mathbf{R}_{3}\right)$ & $g_{1}^{2} g_{3}^{2} Q^{2} I_{2}\left(\mathbf{R}_{3}\right)$ & $\frac{261}{16} g_{1}^{2} g_{3}^{2} Q^{2} I_{2}\left(\mathbf{R}_{3}\right)$ \\
\hline$c_{2}^{B^{2} G^{2}}$ & $\frac{1}{16} g_{1}^{2} g_{3}^{2} Q^{2} I_{2}\left(\mathbf{R}_{3}\right)$ & $\frac{7}{4} g_{1}^{2} g_{3}^{2} Q^{2} I_{2}\left(\mathbf{R}_{3}\right)$ & $\frac{243}{16} g_{1}^{2} g_{3}^{2} Q^{2} I_{2}\left(\mathbf{R}_{3}\right)$ \\
\hline$c_{3}^{B^{2} G^{2}}$ & $\frac{7}{8} g_{1}^{2} g_{3}^{2} Q^{2} I_{2}\left(\mathbf{R}_{3}\right)$ & $2 g_{1}^{2} g_{3}^{2} Q^{2} I_{2}\left(\mathbf{R}_{3}\right)$ & $\frac{261}{8} g_{1}^{2} g_{3}^{2} Q^{2} I_{2}\left(\mathbf{R}_{3}\right)$ \\
\hline$c_{4}^{B^{2} G^{2}}$ & $\frac{1}{8} g_{1}^{2} g_{3}^{2} Q^{2} I_{2}\left(\mathbf{R}_{3}\right)$ & $\frac{7}{2} g_{1}^{2} g_{3}^{2} Q^{2} I_{2}\left(\mathbf{R}_{3}\right)$ & $\frac{243}{8} g_{1}^{2} g_{3}^{2} Q^{2} I_{2}\left(\mathbf{R}_{3}\right)$ \\
\hline$c_{1}^{W^{2} G^{2}}$ & $\frac{7}{16} g_{2}^{2} g_{3}^{2} I_{2}\left(\mathbf{R}_{2}\right) I_{2}\left(\mathbf{R}_{3}\right)$ & $g_{2}^{2} g_{3}^{2} I_{2}\left(\mathbf{R}_{2}\right) I_{2}\left(\mathbf{R}_{3}\right)$ & $\frac{261}{16} g_{2}^{2} g_{3}^{2} I_{2}\left(\mathbf{R}_{2}\right) I_{2}\left(\mathbf{R}_{3}\right)$ \\
\hline$c_{2}^{W^{2} G^{2}}$ & $\frac{1}{16} g_{2}^{2} g_{3}^{2} I_{2}\left(\mathbf{R}_{2}\right) I_{2}\left(\mathbf{R}_{3}\right)$ & $\frac{7}{4} g_{2}^{2} g_{3}^{2} I_{2}\left(\mathbf{R}_{2}\right) I_{2}\left(\mathbf{R}_{3}\right)$ & $\frac{243}{16} g_{2}^{2} g_{3}^{2} I_{2}\left(\mathbf{R}_{2}\right) I_{2}\left(\mathbf{R}_{3}\right)$ \\
\hline$c_{3}^{W^{2} G^{2}}$ & $\frac{7}{8} g_{2}^{2} g_{3}^{2} I_{2}\left(\mathbf{R}_{2}\right) I_{2}\left(\mathbf{R}_{3}\right)$ & $2 g_{2}^{2} g_{3}^{2} I_{2}\left(\mathbf{R}_{2}\right) I_{2}\left(\mathbf{R}_{3}\right)$ & $\frac{261}{8} g_{2}^{2} g_{3}^{2} I_{2}\left(\mathbf{R}_{2}\right) I_{2}\left(\mathbf{R}_{3}\right)$ \\
\hline$c_{4}^{W^{2} G^{2}}$ & $\frac{1}{8} g_{2}^{2} g_{3}^{2} I_{2}\left(\mathbf{R}_{2}\right) I_{2}\left(\mathbf{R}_{3}\right)$ & $\frac{7}{2} g_{2}^{2} g_{3}^{2} I_{2}\left(\mathbf{R}_{2}\right) I_{2}\left(\mathbf{R}_{3}\right)$ & $\frac{243}{8} g_{2}^{2} g_{3}^{2} I_{2}\left(\mathbf{R}_{2}\right) I_{2}\left(\mathbf{R}_{3}\right)$ \\
\hline$c_{1}^{B G^{3}}$ & $\frac{7}{32} g_{1} g_{3}^{3} Q I_{3}\left(\mathbf{R}_{3}\right)$ & $\frac{1}{2} g_{1} g_{3}^{3} Q I_{3}\left(\mathbf{R}_{3}\right)$ & $\frac{261}{32} g_{1} g_{3}^{3} Q I_{3}\left(\mathbf{R}_{3}\right)$ \\
\hline$c_{2}^{B G^{3}}$ & $\frac{1}{32} g_{1} g_{3}^{3} Q I_{3}\left(\mathbf{R}_{3}\right)$ & $\frac{7}{8} g_{1} g_{3}^{3} Q I_{3}\left(\mathbf{R}_{3}\right)$ & $\frac{243}{32} g_{1} g_{3}^{3} Q I_{3}\left(\mathbf{R}_{3}\right)$ \\
\hline
\end{tabular}

Table 5. Wilson coefficients, from ref. [109], for the generalized Euler-Heisenberg operators in table 1 generated by integrating out a massive complex scalar, Dirac fermion, or complex vector charged under the SM gauge group as described in text. All $c_{i}$ have been multiplied by $6 ! \pi^{2}$, and all $c_{i}$ not given in this table vanish at one loop for these completions.

and the $\Lambda$ constants

$$
\begin{aligned}
\Lambda(r)= & \frac{1}{720} r(r+1)(r+2)\left(3 r^{2}+6 r-4\right) \\
\Lambda(p, q)= & \frac{1}{480}(p+1)(q+1)(p+q+2)[p(p+3)+q(q+3)+p q] \\
& \times\left\{\frac{1}{3}[p(p+3)+q(q+3)+p q]-\frac{1}{2}\right\} .
\end{aligned}
$$

In terms of these quantities, the $c_{i}$ as computed for this class of completions are given in table 5. For detailed definitions of these group theoretic quantities, see appendix A. 
The relevant positivity bounds from section 4, which we repeat here for convenience, are

$$
\begin{array}{rlrl}
c_{1}^{B^{4}} & >0 & 3 c_{4}^{G^{4}}+2 c_{6}^{G^{4}} & >0 \\
c_{2}^{B^{4}} & >0 & c_{3}^{B^{2} W^{2}} & >0 \\
c_{1}^{W^{4}}+c_{3}^{W^{4}} & >0 & c_{4}^{B^{2} W^{2}} & >0 \\
c_{2}^{W^{4}}+c_{4}^{W^{4}} & >0 & c_{3}^{B^{2} G^{2}} & >0 \\
3 c_{1}^{G^{4}}+3 c_{3}^{G^{4}}+c_{5}^{G^{4}} & >0 & c_{4}^{B^{2} G^{2}} & >0 \\
3 c_{3}^{G^{4}}+2 c_{5}^{G^{4}} & >0 & c_{3}^{W^{2} G^{2}}>0 \\
3 c_{2}^{G^{4}}+3 c_{4}^{G^{4}}+c_{6}^{G^{4}} & >0 & c_{4}^{W^{2} G^{2}}>0 .
\end{array}
$$

Plugging the $c_{i}$ from table 5 for the scalar, fermion, and vector cases into eq. (5.6), we find that the left-hand sides of the inequalities each become a positive numerical coefficient times one of the following:

$$
g_{1}^{4} Q^{4}, g_{2}^{4} \Lambda\left(\mathbf{R}_{2}\right), g_{3}^{4} \Lambda\left(\mathbf{R}_{3}\right), g_{1}^{2} g_{2}^{2} Q^{2} I_{2}\left(\mathbf{R}_{2}\right), g_{1}^{2} g_{3}^{2} Q^{2} I_{2}\left(\mathbf{R}_{3}\right), \text { or } g_{2}^{2} g_{3}^{2} I_{2}\left(\mathbf{R}_{2}\right) I_{2}\left(\mathbf{R}_{3}\right)
$$

Since $I_{2}\left(\mathbf{R}_{2}\right), I_{2}\left(\mathbf{R}_{3}\right), \Lambda\left(\mathbf{R}_{2}\right)$, and $\Lambda\left(\mathbf{R}_{3}\right)$ are all nonnegative per eqs. (5.3) and (5.5), our bounds are satisfied by this class of completions.

\subsection{Born-Infeld}

Another well-motivated extension of the quartic gauge field operators in table 1 is the BornInfeld (BI) action, in which field strengths have an upper limit. Originally formulated in the context of nonlinear Maxwell theory [112], the BI action appears in string theory models in which the gauge fields are coupled to brane-localized matter (see refs. [113, 114] and refs. therein). The BI action moreover yields amplitudes with structures connected to the gauge/gravity double copy $[100,115]$ and possessing special soft limits [116].

If the SM gauge group is extended to a BI model with universal BI parameter $M^{2}$, then the action is [117]

$$
\mathcal{L}=M^{4}\left[1-\sqrt{1+\frac{1}{2 M^{4}} F_{\mu \nu}^{A} F^{A \mu \nu}-\frac{1}{16 M^{8}}\left(F_{\mu \nu}^{A} \widetilde{F}^{A \mu \nu}\right)^{2}}\right],
$$

where $A$ runs over all twelve generators of $\mathrm{SU}(3) \otimes \mathrm{SU}(2) \otimes \mathrm{U}(1)$, i.e., $F_{\mu \nu}^{A}=\left(B_{\mu \nu}, W_{\mu \nu}^{I}, G_{\mu \nu}^{a}\right)$. Expanding for small field values, we have the EFT up to dimension-eight operators:

$$
\begin{aligned}
\mathcal{L}= & -\frac{1}{4} B_{\mu \nu} B^{\mu \nu}-\frac{1}{4} W_{\mu \nu}^{I} W^{I \mu \nu}-\frac{1}{4} G_{\mu \nu}^{a} G^{a \mu \nu} \\
& +\frac{1}{32 M^{4}}\left(\mathcal{O}_{1}^{B^{4}}+\mathcal{O}_{2}^{B^{4}}+\mathcal{O}_{1}^{W^{4}}+\mathcal{O}_{2}^{W^{4}}+\mathcal{O}_{1}^{G^{4}}+\mathcal{O}_{2}^{G^{4}}\right) \\
& +\frac{1}{16 M^{4}}\left(\mathcal{O}_{1}^{B^{2} W^{2}}+\mathcal{O}_{2}^{B^{2} W^{2}}+\mathcal{O}_{1}^{B^{2} G^{2}}+\mathcal{O}_{2}^{B^{2} G^{2}}+\mathcal{O}_{1}^{W^{2} G^{2}}+\mathcal{O}_{2}^{W^{2} G^{2}}\right) .
\end{aligned}
$$

Reading off the $c_{i}$, we see that the bounds in eq. (5.6) are manifestly satisfied. 


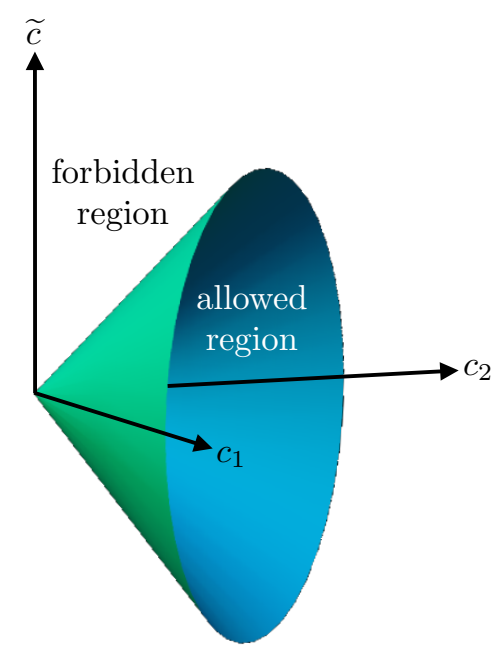

Figure 5. General form of the bounds derived in section 4. CP-conserving terms (or linear combinations thereof) have coefficients $c_{1}$ and $c_{2}$ bounded to be positive, while the corresponding CP-breaking term has coefficient $\widetilde{c}$ satisfying $\widetilde{c}^{2}<4 c_{1} c_{2}$.

We could also consider a different BI action, with different BI parameters $M_{i}^{2}$,

$$
\begin{aligned}
\mathcal{L}= & M_{1}^{4}\left[1-\sqrt{1+\frac{1}{2 M_{1}^{4}} B_{\mu \nu} B^{\mu \nu}-\frac{1}{16 M_{1}^{8}}\left(B_{\mu \nu} \widetilde{B}^{\mu \nu}\right)^{2}}\right] \\
& +M_{2}^{4}\left[1-\sqrt{1+\frac{1}{2 M_{2}^{4}} W_{\mu \nu}^{I} W^{I \mu \nu}-\frac{1}{16 M_{2}^{8}}\left(W_{\mu \nu}^{I} \widetilde{W}^{I \mu \nu}\right)^{2}}\right] \\
& +M_{3}^{4}\left[1-\sqrt{1+\frac{1}{2 M_{3}^{4}} G_{\mu \nu}^{a} G^{a \mu \nu}-\frac{1}{16 M_{3}^{8}}\left(G_{\mu \nu}^{a} \widetilde{G}^{a \mu \nu}\right)^{2}}\right]
\end{aligned}
$$

for which the EFT becomes

$$
\begin{aligned}
\mathcal{L}= & -\frac{1}{4} B_{\mu \nu} B^{\mu \nu}-\frac{1}{4} W_{\mu \nu}^{I} W^{I \mu \nu}-\frac{1}{4} G_{\mu \nu}^{a} G^{a \mu \nu} \\
& +\frac{1}{32 M_{1}^{4}} \mathcal{O}_{1}^{B^{4}}+\frac{1}{32 M_{1}^{4}} \mathcal{O}_{2}^{B^{4}}+\frac{1}{32 M_{2}^{4}} \mathcal{O}_{1}^{W^{4}}+\frac{1}{32 M_{2}^{4}} \mathcal{O}_{2}^{W^{4}}+\frac{1}{32 M_{3}^{4}} \mathcal{O}_{1}^{G^{4}}+\frac{1}{32 M_{3}^{4}} \mathcal{O}_{2}^{G^{4}},
\end{aligned}
$$

which also clearly satisfies the bounds in eq. (5.6).

\subsection{CP-violating terms and completing the square}

A striking feature of the bounds derived in section 4 is that they come in two distinct forms, either $c>0$, for some CP-even term with coefficient $c$, or $\widetilde{c}^{2}<4 c_{1} c_{2}$, where $\widetilde{c}$ is the coefficient of a CP-odd term and $c_{1,2}$ are the positive coefficients of CP-even terms. In other words, the bounds take the form of a cone, $\widetilde{c}^{2}+c_{-}^{2}<c_{+}^{2}$ for $c_{+}>0$, where $c_{ \pm}=c_{1} \pm c_{2}$. That is, the overall size of CP-odd terms tends to be upper-bounded by the size of CP-even terms appearing in the action; see figure 5 .

Let us restrict our attention to the $\mathcal{O}_{i}^{B^{4}}$ terms containing four $\mathrm{U}(1)$ field strengths. The bounds in eq. (4.18), $c_{1,2}^{B^{4}}>0$ and $\left(\widetilde{c}_{1}^{B^{4}}\right)^{2}<4 c_{1}^{B^{4}} c_{2}^{B^{4}}$, imply that it is possible to rewrite 


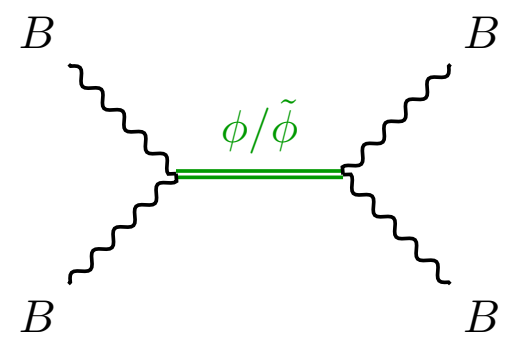

Figure 6. Tree-level interaction between four $\mathrm{U}(1)_{Y}$ gauge bosons, mediated by a CP-even scalar $\phi$, a CP-odd scalar $\widetilde{\phi}$, and their mixing. When these states are integrated out, CP even and odd $B^{4}$ operators are generated, and their coefficients satisfy our bound on the CP-odd coefficient given in eq. (4.18), in detail $\left(\tilde{c}_{1}^{B^{4}}\right)^{2}<4 c_{1}^{B^{4}} c_{2}^{B^{4}}$.

the Lagrangian corrections

$$
\Delta \mathcal{L}=\frac{1}{M^{4}}\left[c_{1}^{B^{4}}\left(B_{\mu \nu} B^{\mu \nu}\right)^{2}+c_{2}^{B^{4}}\left(B_{\mu \nu} \widetilde{B}^{\mu \nu}\right)^{2}+\widetilde{c}_{1}^{B^{4}} B_{\mu \nu} B^{\mu \nu} B_{\rho \sigma} \widetilde{B}^{\rho \sigma}\right]
$$

as a sum of perfect squares,

$$
\Delta \mathcal{L}=\frac{\alpha^{2}}{2 M^{4}}\left[\left(B_{\mu \nu} B^{\mu \nu}+\beta B_{\mu \nu} \widetilde{B}^{\mu \nu}\right)^{2}+\gamma^{2}\left(B_{\mu \nu} B^{\mu \nu}-\beta B_{\mu \nu} \widetilde{B}^{\mu \nu}\right)^{2}\right],
$$

where $\alpha, \beta, \gamma$ are real constants chosen such that $\alpha^{2}\left(1+\gamma^{2}\right)=2 c_{1}^{B^{4}}, \alpha^{2} \beta^{2}\left(1+\gamma^{2}\right)=2 c_{2}^{B^{4}}$, and $\alpha^{2} \beta\left(1-\gamma^{2}\right)=\widetilde{c}_{1}^{B^{4}}$, so $\left(\widetilde{c}_{1}^{B^{4}}\right)^{2} / 4 c_{1}^{B^{4}} c_{2}^{B^{4}}=\left[\left(1-\gamma^{2}\right) /\left(1+\gamma^{2}\right)\right]^{2}$ and eq. (4.18) guarantees that such a choice of $\alpha, \beta, \gamma \in \mathbb{R}$ exists.

The sum-of-squares form of the action in eq. (5.13) suggests how it can be generated at tree level through mass mixing of a massive dilaton and axion, ${ }^{19}$ coupling a CP-even scalar $\phi$ and CP-odd scalar $\widetilde{\phi}$ to $B_{\mu \nu} B^{\mu \nu}$ and $B_{\mu \nu} \widetilde{B}^{\mu \nu}$, respectively: ${ }^{20}$

$$
\mathcal{L} \supset-\frac{M^{2}}{2}(\phi+\widetilde{\phi})^{2}-\frac{M^{2}}{2 \gamma^{2}}(\phi-\widetilde{\phi})^{2}+\frac{2 \alpha}{M} \phi B_{\mu \nu} B^{\mu \nu}+\frac{2 \alpha \beta}{M} \widetilde{\phi} B_{\mu \nu} \widetilde{B}^{\mu \nu},
$$

where $\phi+\widetilde{\phi}$ and $\phi-\widetilde{\phi}$ are the mass eigenstates, with masses $M$ and $M /|\gamma|$, respectively. (When $\gamma=1$ the two masses are degenerate, the mass mixing between $\phi$ and $\widetilde{\phi}$ vanishes, and the CP-odd coefficient $\widetilde{c}_{1}^{B^{4}}=0$.) Integrating out $\phi$ and $\widetilde{\phi}$, effectively replacing the diagram in figure 6 by a quartic $B$ vertex, we obtain the interactions in eq. (5.13). Indeed, any scalar tree-level completion of $\mathcal{O}_{1,2}^{B^{4}}$ and $\widetilde{\mathcal{O}}_{1}^{B^{4}}$ will manifestly obey the bounds in eq. (4.18):

\footnotetext{
${ }^{19}$ Such a model may be motivated by string theory, where the universal axion and dilaton can be treated as real and imaginary parts of a complex modular parameter [118-120], though difficulties remain in engineering mass splitting [121].

${ }^{20}$ As this Lagrangian involves dimension-five operators, it is not a complete UV theory. However, it is still a UV extension of the theory written in terms of dimension-eight operators, in that its amplitudes will grow more slowly with momentum and its cutoff will be higher if the theory is weakly coupled; focusing on the $\alpha$ coefficient, the cutoff of the original EFT in eq. (5.13) is at $\sim M / \sqrt{\alpha}$, while that of the theory in eq. (5.14) is at $\sim M / \alpha$. The latter action can be itself completed in any of the ways theories of axions and dilatons traditionally are without impacting the arguments in this section. A similar comment applies for the completions considered in section 5.4.
} 


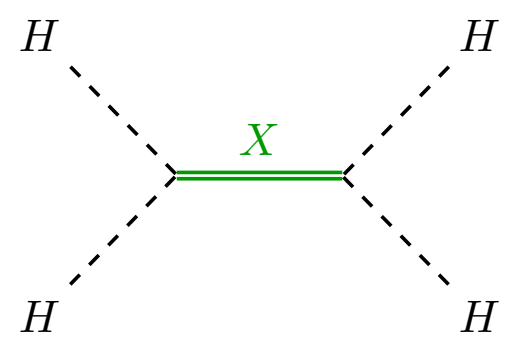

Figure 7. Tree-level completion of a $(D H)^{4}$ operator, where $X$ is a heavy field transforming under a specific representation of $\mathrm{SU}(2)_{L}$. In the text, we consider a number of examples for $X$ and show in all cases that, when it is integrated out, the coefficients of the $(D H)^{4}$ operators generated always satisfy our bounds.

$c_{1,2}^{B^{4}}$ will both be positive when integrating out a nontachyonic state coupled at tree level to $B_{\mu \nu} B^{\mu \nu}$ and $B_{\mu \nu} \widetilde{B}^{\mu \nu}$, respectively, while $\widetilde{c}_{1}^{B^{4}}$ can be generated by a scalar at tree level only through a state that breaks $\mathrm{CP}$ by simultaneously coupling to $B_{\mu \nu} B^{\mu \nu}$ and $B_{\mu \nu} \widetilde{B}^{\mu \nu}$, generating $\widetilde{O}_{1}^{B^{4}}$ through the cross term but also generating $O_{1,2}^{B^{4}}$. Beyond the example completion in eq. (5.14), it would be interesting to understand, from a UV perspective and for more general completions, why CP-violating terms in the EFT are constrained to always come in the form of sums of perfect squares with CP-even terms. For example, a similar pattern of bounds was found from causality for CP-even and -odd quartic Riemann operators in ref. [122]. We leave such investigations to future work.

\subsection{Tree-level completions of $(\mathrm{DH})^{4}$ operators}

Finally, let us consider the three $(D H)^{4}$ operators $\mathcal{O}_{1,2,3}^{H^{4}}$ given in table 2,

$$
\begin{aligned}
& \mathcal{O}_{1}^{H^{4}}=\left(D_{\mu} H^{\dagger} D_{\nu} H\right)\left(D^{\nu} H^{\dagger} D^{\mu} H\right) \\
& \mathcal{O}_{2}^{H^{4}}=\left(D_{\mu} H^{\dagger} D_{\nu} H\right)\left(D^{\mu} H^{\dagger} D^{\nu} H\right) \\
& \mathcal{O}_{3}^{H^{4}}=\left(D^{\mu} H^{\dagger} D_{\mu} H\right)\left(D^{\nu} H^{\dagger} D_{\nu} H\right) .
\end{aligned}
$$

We will construct a few representative completions of these operators via the tree-level exchange of massive states, as depicted in figure 7, and verify that they obey the three bounds derived in eq. (4.30), $c_{2}^{H^{4}}>0, c_{1}^{H^{4}}+c_{2}^{H^{4}}>0$, and $c_{1}^{H^{4}}+c_{2}^{H^{4}}+c_{3}^{H^{4}}>0$.

First, the simplest example tree completion of a $(D H)^{4}$ operator is a massive real scalar $\phi$ transforming as a singlet under $\mathrm{SU}(2)$, with UV action

$$
\mathcal{L} \supset-D^{\mu} H^{\dagger} D_{\mu} H-\frac{1}{2}(\partial \phi)^{2}-\frac{1}{2} m^{2} \phi^{2}+\frac{\alpha}{M} \phi\left(D^{\mu} H^{\dagger} D_{\mu} H\right),
$$

where $\alpha$ is an arbitrary real coupling. Integrating out $\phi$ generates the Wilson coefficients

$$
\left.\left(c_{1}^{H^{4}}, c_{2}^{H^{4}}, c_{3}^{H^{4}}\right)=\frac{\alpha^{2}}{2 m^{2} M^{2}}(0,0,1) . \quad \text { [singlet scalar }\right]
$$

Second, let us consider completion via a massive state $\pi^{I}$ that transforms as an $\mathrm{SU}(2)$ triplet:

$$
\mathcal{L} \supset-D^{\mu} H^{\dagger} D_{\mu} H-\frac{1}{2} \partial^{\mu} \pi^{I} \partial_{\mu} \pi^{I}-\frac{1}{2} m^{2} \pi^{I} \pi^{I}+\frac{\alpha}{M} \pi^{I}\left(D^{\mu} H^{\dagger} \tau^{I} D_{\mu} H\right) .
$$


Using the Pauli matrix completeness relation,

$$
\left(\sigma^{I}\right)_{i}^{j}\left(\sigma^{I}\right)_{k}^{l}=2 \delta_{i}^{l} \delta_{k}^{j}-\delta_{i}^{j} \delta_{k}^{l},
$$

one finds that at low energies $\pi^{I}$ generates the coefficients

$$
\left.\left(c_{1}^{H^{4}}, c_{2}^{H^{4}}, c_{3}^{H^{4}}\right)=\frac{\alpha^{2}}{8 m^{2} M^{2}}(2,0,-1) . \quad \text { [triplet scalar }\right]
$$

Third, let us consider a complex bifundamental scalar $\rho_{i j}$ transforming as a $\mathbf{2} \otimes \mathbf{2}$ of $\mathrm{SU}(2)$. In analogy with $H$ and $H^{\dagger}$ in eqs. (3.10) and (3.11), we can write out $\rho_{i j}$ and $\rho_{i j}^{\dagger}$ in terms of real fields $\sigma_{1, \ldots, 8}{ }^{21}$

$$
\rho_{i j}=\frac{1}{\sqrt{2}}\left(\begin{array}{cc}
\sigma_{1}+i \sigma_{2} & \sigma_{3}+i \sigma_{4} \\
\sigma_{5}+i \sigma_{6} & \sigma_{7}+i \sigma_{8}
\end{array}\right) \quad \text { and } \quad \rho_{i j}^{\dagger}=\frac{1}{\sqrt{2}}\left(\begin{array}{cc}
\sigma_{7}-i \sigma_{8} & -\sigma_{5}+i \sigma_{6} \\
-\sigma_{3}+i \sigma_{4} & \sigma_{1}-i \sigma_{2}
\end{array}\right)
$$

and define $\rho^{i j}=\epsilon^{i k} \epsilon^{j l} \rho_{k l}$ and $\rho^{\dagger i j}=\epsilon^{i k} \epsilon^{j l} \rho_{k l}^{\dagger}$. Let us then consider the action

$$
\mathcal{L} \supset-D^{\mu} H^{\dagger} D_{\mu} H-\partial^{\mu} \rho_{i j}^{\dagger} \partial_{\mu} \rho^{i j}-m^{2} \rho_{i j}^{\dagger} \rho^{i j}+\frac{1}{M}\left(\alpha D^{\mu} H_{i}^{\dagger} \rho^{i j} D_{\mu} H_{j}^{\dagger}+\alpha^{*} D^{\mu} H^{i} \rho_{i j}^{\dagger} D_{\mu} H^{j}\right) .
$$

Since $\rho_{i j}^{\dagger} \rho^{i j}=+\frac{1}{2} \sum_{n=1}^{8} \sigma_{n}^{2}$, the action contains no ghosts or tachyons. Here, $\alpha$ is an arbitrary complex unitless coupling, and one van verify that the interaction term is selfhermitian. Integrating out $\rho_{i j}$, we obtain the EFT coefficients

$$
\left.\left(c_{1}^{H^{4}}, c_{2}^{H^{4}}, c_{3}^{H^{4}}\right)=\frac{|\alpha|^{2}}{m^{2} M^{2}}(0,1,0) . \quad \text { [bifundamental scalar }\right]
$$

A final well-motivated example completion is a massive symmetric spin-two state $\chi_{\mu \nu}$, coupled to the Higgs stress tensor $T_{\mu \nu}$ akin to a massive graviton (as would naturally occur in, e.g., a Kaluza-Klein model):

$$
\mathcal{L} \supset-D^{\mu} H^{\dagger} D_{\mu} H+\mathcal{L}_{\mathrm{FP}}+\frac{\alpha}{M} \chi^{\mu \nu} T_{\mu \nu},
$$

where $\mathcal{L}_{\mathrm{FP}}$ is the Fierz-Pauli action [123], $\alpha$ is real, and we can take $T_{\mu \nu}=D_{\mu} H^{\dagger} D_{\nu} H+$ $D_{\nu} H^{\dagger} D_{\mu} H-g_{\mu \nu}\left(D^{\rho} H^{\dagger} D_{\rho} H\right)$, neglecting Higgs mass and potential terms as we are interested in specifically generating $\mathcal{O}_{1,2,3}^{H^{4}}$ enumerated above. Integrating out $\chi_{\mu \nu}$, we obtain the EFT operators with coefficients

$$
\left.\left(c_{1}^{H^{4}}, c_{2}^{H^{4}}, c_{3}^{H^{4}}\right)=\frac{\alpha^{2}}{3 m^{2} M^{2}}(3,3,-2) . \quad \text { [symmetric tensor }\right]
$$

The bounds in eq. (4.30) delineate a the triangular cone defining the allowed space of $c_{1,2,3}^{H^{4}}$; see figure 8. We find, as required, that the sets of Wilson coefficients explored in the examples in eqs. (5.17), (5.20), (5.23), and (5.25) correspond to vectors lying either on the edge of or interior to this cone.

\footnotetext{
${ }^{21} \mathrm{As}$ in footnote 10, we again use the $\mathrm{SU}(2)$ notation conventions of ref. [87], where each lowered index of $\rho_{i j}^{\dagger}$ is treated as a $\mathbf{2}$ rather than a $\overline{\mathbf{2}}$.
} 


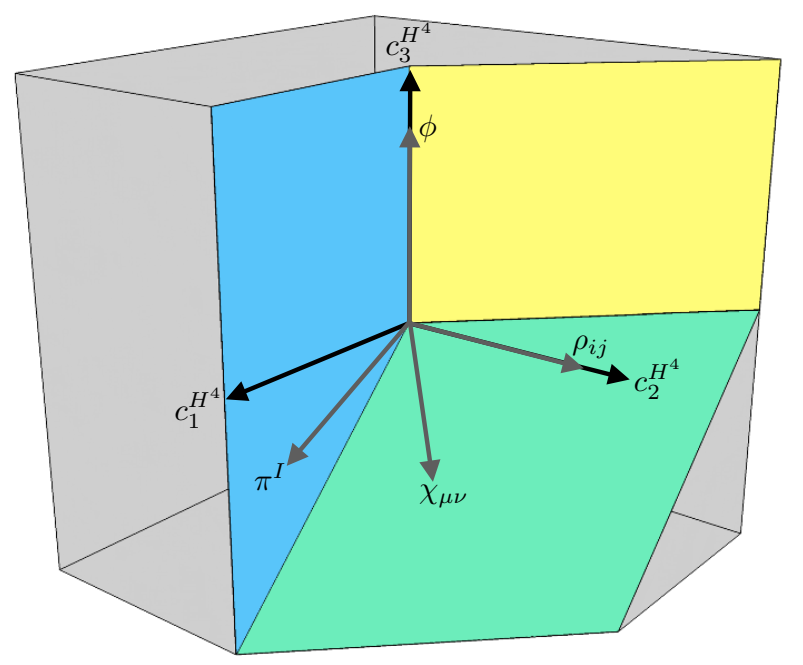

Figure 8. Bounds on the three $(D H)^{4}$ operator coefficients from eq. (4.30): $c_{2}^{H^{4}}>0$ (blue), $c_{1}^{H^{4}}+c_{2}^{H^{4}}>0$ (yellow), and $c_{1}^{H^{4}}+c_{2}^{H^{4}}+c_{3}^{H^{4}}>0$ (green). Gray arrows indicate the vectors of Wilson coefficients generated in example tree-level completions, all lying in the interior or on the boundary of the allowed space, with various massive states coupling to $(D H)^{2}$ : a scalar singlet $\phi$, scalar triplet $\pi^{I}$, bifundamental scalar $\rho_{i j}$, and symmetric spin-two field $\chi_{\mu \nu}$. The forbidden region is shaded in gray.

\section{Phenomenological consequences}

Searching for deviations from the SM is one of the driving forces for all of particle physics. The bounds derived in this work demarcate the space of experimentally accessible deviations into those that can be associated with well-defined UV completions and those that cannot. Accordingly, the bounds represent significantly constraining theoretical priors on the SMEFT parameter space, which would be particularly appropriate in the context of global analyses $[124,125]$. Conversely, any conclusively-measured experimental violation of our bounds would have powerful implications for fundamental assumptions about physics, falsifying low-energy causality/locality, Lorentz invariance, and, by extension, both lowenergy quantum field theory and perturbative string theory [74]. Performing such a direct test of our bounds is a challenging endeavor. Not only does it require a discovery of new physics generated by the SMEFT, for many of our bounds a definitive determination of the sign as well as the magnitude is required. ${ }^{22}$ In spite of the challenges, the possibility to test the fundamental pillars of modern physics represents a unique motivation for experiments sensitive to the operators we bound.

The full reach of experimental effects associated with the operators considered in this work is vast. Given this scope, in this section we will restrict ourselves to a brief exploration of searches where our bounds have immediate application. One example of such a search is the measurement of aQGCs at the LHC. Such searches are expected to represent a viable

\footnotetext{
${ }^{22}$ Extracting the sign of the operator requires an observable linear in the operator coefficient. Some observables, such as EDMs, are automatically linear in the coefficient, but for others extracting the sign will require interference with an associated SM contribution.
} 
discovery channel for several of the operators we have considered, making our bounds immediately relevant. Beyond aQGCs, we also outline how our bounds are likely to have consequences for additional collider searches, as well as other precision probes such as neutron EDM measurements.

As demonstrated in section 5, a generic UV completion will satisfy our bounds. For this reason, we do not see our results as immediate probes of the viable BSM model building space. To take an example, a theory where dark matter is made up of bound states of color octet fermions [126] will automatically satisfy our bounds, as shown in section 5.1. Instead, our bounds are most applicable in the context of the SMEFT, which will be the focus of this section.

Before presenting explicit examples, we mention a caveat that must be kept in mind whenever constraints on the SMEFT are being considered. Whenever a limit on the scale $M$ of higher-dimension operators is quoted, one must ensure that the limit was derived within the regime of validity for the EFT. In particular, if the measurement was performed at an invariant mass scale $\sqrt{s}$, then unless $\sqrt{s}<M$, the EFT limit was derived in a region where the theory violated perturbative unitarity, and can no longer be trusted. This point is often relevant for SMEFT constraints at the LHC — for a few examples see refs. [127-131] - but must always be considered when EFT constraints are discussed.

\subsection{Anomalous quartic gauge-boson couplings}

Through a subset of the operators we have considered, BSM physics can induce anomalous corrections to the tree-level SM quartic gauge-boson couplings $W W W W, W W Z Z$, $W W \gamma \gamma$, and $W W Z \gamma$ or even induce vertices not observed in the SM at tree level such as $Z Z Z Z$. The set of all such processes are collectively referred to as aQGCs. ${ }^{23}$ Importantly, the LHC can probe these vertices and, accordingly, the corrections that dimension-eight operators induce. For example, the $W W W W$ and $W W Z Z$ vertices can be probed using channels such as $q q \rightarrow q q W W$ and $q q \rightarrow q q Z Z$. This is illustrated in figure 9 for an example Feynman diagram contributing to these processes, where dimension-eight operators can provide corrections to the highlighted four-point vertex.

As pointed out in ref. [76], aQGC searches represent a powerful channel for probing a number of dimension-eight SMEFT operators. Naively, one may worry that any effects will be swamped by the contribution from the dimension-six bosonic operators shown in table 3, which, when squared, will also contribute to aQGCs. Yet a subset of these dimension-six operators also induce anomalous triple gauge-boson couplings, on which the LHC has significantly extended the constraints from LEP [133] (see also ref. [134]), potentially leaving room for aQGCs from dimension-eight operators. Deviations from the SM in these channels have been searched for at both ATLAS [27-29] and CMS [30-32]; for more detail see refs. $[75,135,136]$. At the moment no anomalies have been observed, and assuming $\mathcal{O}(1)$ couplings, these searches are currently probing new physics scales from the hundreds of $\mathrm{GeV}$ to $\mathrm{TeV}$ range. Importantly, however, if a deviation from the $\mathrm{SM}$ were to

\footnotetext{
${ }^{23}$ Additional quartic gauge couplings that are not always considered within the aQGC framework can also be probed at the LHC. For example, constraints on dimension-eight operators associated with $\gamma \gamma \rightarrow \gamma \gamma$ and $g g \rightarrow \gamma \gamma$ have been considered in the literature [117, 132], although we will not discuss them further.
} 


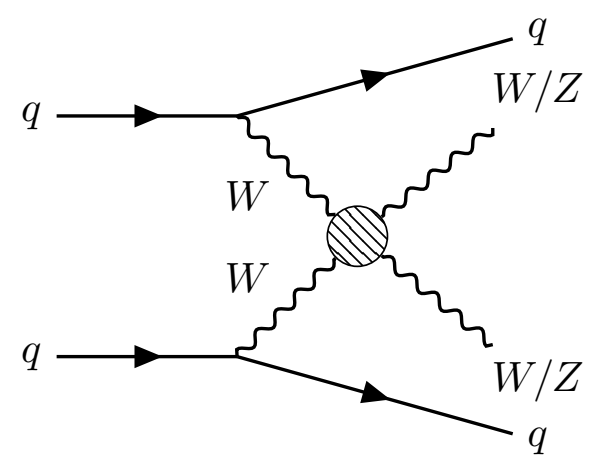

Figure 9. Example Feynman diagram for the contribution of aQGCs to the $q q \rightarrow q q W W$ or $q q \rightarrow q q Z Z$ channel.

emerge in these channels, it could represent a sign of new physics first appearing through dimension-eight operators.

In addition to pointing out the importance of aQGCs for dimension-eight operators, ref. [76] also introduced the commonly-used set of operators for these effects. This list, which has since received several corrections (see, for example, ref. [136] for details), contains $18 \mathrm{CP}$-even operators, all of which are related to operators we considered above. Conventionally, the 18 are divided into three types of operators:

1. 3 scalar or S-type operators of the form $(D H)^{4}$,

2. 7 mixed or M-type operators, schematically $(D H)^{2} F^{2}$, and

3. 8 tensor or T-type operators, such as $F^{4}$.

As aQGCs exclude gluonic vertices, in the above $F$ can be $B$ or $W$. A complete mapping between this notation and the basis of operators used in the present work is possible and will be provided below. We note in passing that our basis for such processes includes three additional independent operators that are missing from the standard aQGC list. In detail, while we also have three operators of the form $(D H)^{4}$, as shown in table 2 we have 8 M-type operators such as $(D H)^{2} B^{2},(D H)^{2} W^{2}$, or $(D H)^{2}(B W)$, and we have 10 T-type operators as can be seen in table 1 . One can show that all the operators in our basis are indeed independent [86], so the aQGC operator basis commonly used in the literature is incomplete. A complete basis is crucial, since an incomplete operator basis could in principle lead to conflation and/or overlooking of distinct signals of new physics.

Before converting our bounds into the conventional aQGC language, let us comment on the related work in refs. $[65,66]$, which also considered the question of positivity constraints on these operators. Those works determined the restrictions associated with the forward limit of all possible $2 \rightarrow 2$ electroweak boson scatterings, such as $\gamma \gamma \rightarrow \gamma \gamma$ or $\gamma W^{ \pm} \rightarrow \gamma W^{ \pm}$. This can be contrasted with the approach we have taken in this work, where we have restricted our attention to the scattering of bosons in the limit of unbroken electroweak symmetry; this choice is natural for theories with completions above the electroweak scale, and we accordingly organized our SMEFT operators in the unbroken basis using $B$ and 
$W^{I}$. In ref. [65], the authors considered 18 aQGC operators, with the results in their table 1 stated using a one-at-a-time approach, where limits are derived on a single operator assuming all others vanish. The work of ref. [65] also considered the more general case where multiple operators at a time are allowed to be nonzero, the results of which were then expanded upon significantly in ref. [66]. Given the different starting points used to derive our bounds, as contrasted to those in ref. [65], instead of comparing each of our full expressions, we will consider just results restricted to a single operator and show that our bounds are generally in agreement.

We caution that the one-at-a-time approach can lead to results that must be interpreted carefully. Indeed, unlike our bounds, which we exhaustively verified in section 5 to be consistent with a large suite of UV completions, bounds derived in a one-at-a-time approach will generically be violated by these checks. We will provide several explicit examples of this below. The loophole is that the bounds are always violated in a context where at least one additional operator is also generated, implying a contradiction with the basic one-ata-time assumption. This highlights that one-at-a-time bounds are not generic conditions that must be satisfied by any UV completion, but rather conditions that can only possibly apply to particular high-scale theories that, when the UV degrees of freedom are integrated out, generate very simple IR EFTs. As we saw in section 5, such completions are highly nongeneric. Even for those theories where this assumption is satisfied, however, below the matching scale renormalization-group evolution will also generically induce operator mixing, and therefore, at the relevant experimental scale, invalidate the single-operator assumption. For such reasons, one-at-a-time results must be interpreted carefully, and often have a narrow range of applicability.

Such theoretical considerations do not immediately address the practical consideration underlying the one-at-a-time approach: it is not straightforward to determine, and then present in a useful manner, limits on the 18-dimensional space spanned by the aQGC operators. Fundamentally, it is a challenge to find enough observables with sufficient statistics to disentangle the various operators. Motivated by this concern, experimental limits are almost exclusively quoted on single operators, although there have also been efforts to extend this to two operators; see for example ref. [32]. In spite of this, theoretical consistency only requires that the complete basis of appropriate operators be considered when deriving the bounds; as we have seen many times above, individual bounds themselves usually only apply to a significantly smaller subset of operators. The same will be true when our bounds are converted to the space of aQGC operators. We will find many examples of bounds that apply to a single operators, or to small numbers of operators, and, unlike for one-at-a-time results, our bounds apply to all possible UV completions.

With the above considerations in mind, let us return to converting our operators and bounds into the conventional notation of aQGCs, considering the S-type, M-type, and T-type operators in turn. To begin with, the three S-type operators in the notation of refs. $[65,66,136]$ map directly onto our $(D H)^{4}$ operators given in table 2 as follows:

$$
\mathcal{O}_{S, 0}=\mathcal{O}_{2}^{H^{4}}, \quad \mathcal{O}_{S, 1}=\mathcal{O}_{3}^{H^{4}}, \quad \text { and } \quad \mathcal{O}_{S, 2}=\mathcal{O}_{1}^{H^{4}}
$$


Using this mapping, our constraints on these operators given in eq. (4.30) become

$$
\begin{aligned}
c_{S, 0}+c_{S, 1}+c_{S, 2} & >0 \\
c_{S, 0}+c_{S, 2} & >0 \\
c_{S, 0} & >0 .
\end{aligned}
$$

These results can be contrasted with the one-at-a-time bounds derived in table 1 of ref. [65], which required that each of these coefficients be individually positive. Looking back at the results in section 5.4, we can see that two of the four example completions discussed in that section have one of these coefficients negative. These same two completions also generate multiple operators, so these provide examples of the inconsistency associated with interpreting operator bounds on a one-at-a-time basis.

Turning to the M-type operators, our bounds impact three of the seven operators in the commonly-used partial aQGC basis. Those operators, in the notation of refs. [65, 66, 136], can be converted to our basis in table 2 using

$$
\begin{aligned}
\mathcal{O}_{M, 0} & =-\frac{g_{2}^{2}}{2} \mathcal{O}_{2}^{H^{2} W^{2}} \\
\mathcal{O}_{M, 1} & =\frac{g_{2}^{2}}{2} \mathcal{O}_{1}^{H^{2} W^{2}} \\
\mathcal{O}_{M, 2} & =-\frac{g_{1}^{2}}{4} \mathcal{O}_{2}^{H^{2} B^{2}} \\
\mathcal{O}_{M, 3} & =\frac{g_{1}^{2}}{4} \mathcal{O}_{1}^{H^{2} B^{2}} \\
\mathcal{O}_{M, 4} & =-\frac{g_{1} g_{2}}{2} \mathcal{O}_{1}^{H^{2} B W} \\
\mathcal{O}_{M, 5} & =\frac{g_{1} g_{2}}{4}\left(i \mathcal{O}_{2}^{H^{2} B W}-\mathcal{O}_{3}^{H^{2} B W}\right) \\
\mathcal{O}_{M, 7} & =\frac{g_{2}^{2}}{4}\left(2 \mathcal{O}_{3}^{H^{2} W^{2}}-\mathcal{O}_{1}^{H^{2} W^{2}}\right),
\end{aligned}
$$

where $g_{1,2}$ are the $\mathrm{U}(1)_{Y}$ and $\mathrm{SU}(2)_{L}$ couplings, respectively. Accordingly, our two relevant bounds in eq. (4.33) convert to a requirement that

$$
\begin{aligned}
2 c_{M, 1}-c_{M, 7} & >0 \\
c_{M, 3} & >0 .
\end{aligned}
$$

Recall that the overall sign of these operators, and hence the bounds, varies between metric conventions. After accounting for the fact we use the mostly-plus convention in this work, these bounds are also consistent with those in ref. [65].

Finally, for the T-type operators, we will make use of the following conversion from the notation of refs. $[65,66,136]$ to a subset of the operators in our basis, as listed in table 1 :

$$
\begin{aligned}
& \mathcal{O}_{T, 0}=\frac{g_{2}^{4}}{4} \mathcal{O}_{1}^{W^{4}} \\
& \mathcal{O}_{T, 1}=\frac{g_{2}^{4}}{4} \mathcal{O}_{3}^{W^{4}}
\end{aligned}
$$




$$
\begin{aligned}
\mathcal{O}_{T, 2} & =\frac{g_{2}^{4}}{16}\left(\mathcal{O}_{1}^{W^{4}}+\mathcal{O}_{3}^{W^{4}}+\mathcal{O}_{4}^{W^{4}}\right) \\
\mathcal{O}_{T, 5} & =\frac{g_{1}^{2} g_{2}^{2}}{8} \mathcal{O}_{1}^{B^{2} W^{2}} \\
\mathcal{O}_{T, 6} & =\frac{g_{1}^{2} g_{2}^{2}}{8} \mathcal{O}_{3}^{B^{2} W^{2}} \\
\mathcal{O}_{T, 7} & =\frac{g_{1}^{2} g_{2}^{2}}{32}\left(\mathcal{O}_{1}^{B^{2} W^{2}}+\mathcal{O}_{3}^{B^{2} W^{2}}+\mathcal{O}_{4}^{B^{2} W^{2}}\right) \\
\mathcal{O}_{T, 8} & =\frac{g_{1}^{4}}{16} \mathcal{O}_{1}^{B^{4}} \\
\mathcal{O}_{T, 9} & =\frac{g_{1}^{4}}{64}\left(2 \mathcal{O}_{1}^{B^{4}}+\mathcal{O}_{2}^{B^{4}}\right) .
\end{aligned}
$$

Our constraints given in eqs. (4.16), (4.18), and (4.22) then imply

$$
\begin{aligned}
2 c_{T, 0}+2 c_{T, 1}+c_{T, 2} & >0 \\
4 c_{T, 6}+c_{T, 7} & >0 \\
c_{T, 7} & >0 \\
2 c_{T, 8}+c_{T, 9} & >0 \\
c_{T, 9} & >0 .
\end{aligned}
$$

Moreover, our bound $c_{2}^{W^{4}}+c_{4}^{W^{4}}>0$ from eq. (4.16) cannot be converted into aQGC notation, as the conventional aQGC basis is incomplete, missing the operator $\mathcal{O}_{2}^{W^{4}}$. The majority of the T-type bounds in eq. (6.6) are consistent with those in table 1 of ref. [65]. The only exception is that we require $c_{T, 7}>0$, whereas when those authors restricted their results to a single operator, they found $c_{T, 7}=0$. In that work, the authors also required that the coefficient of $\mathcal{O}_{T, 5}$, or equivalently our $\mathcal{O}_{1}^{B^{2} W^{2}}$, vanished. Looking back to the relevant row in table 5, we see that any one-loop completion of these operators that involves a scalar, fermion, or vector that is charged under both $\mathrm{SU}(2)_{L}$ and $\mathrm{U}(1)_{Y}$ will generate a nonzero coefficient for both $\mathcal{O}_{T, 5}$ and $\mathcal{O}_{T, 7}$, in conflict with the one-at-a-time bounds in ref. [65]. These completions are standard quantum field theories and are analogous to the completion of the Euler-Heisenberg action by the electron loop in quantum electrodynamics [109], so these models should not be forbidden by any consistent set of positivity bounds, providing another explicit example of the limitations of one-at-a-time results.

Having converted our bounds into the aQGC notation, we see from eqs. (6.2), (6.4), and (6.6) that we are able to demand the positivity of four of the 18 commonly-used aQGC couplings individually, with a further five coefficients entering bounds containing two operators and an additional four coefficients entering only bounds containing three operators. Two depictions of the allowed space are shown in figure 10 and represent two-dimensional views of the parameter space where the unseen parameters have been marginalized over. As we derived the bounds while considering all possible four-fieldstrength operators, these constraints must be satisfied by any consistent UV completion. Nevertheless, while our bounds apply to every UV completion, not every UV completion will generate only the combinations of operator coefficients that appear in our bounds. This point is manifest in table 5 , where we see that introducing a new field at one loop that is charged under the SM will generically yield a large number of higher-dimension operators. 

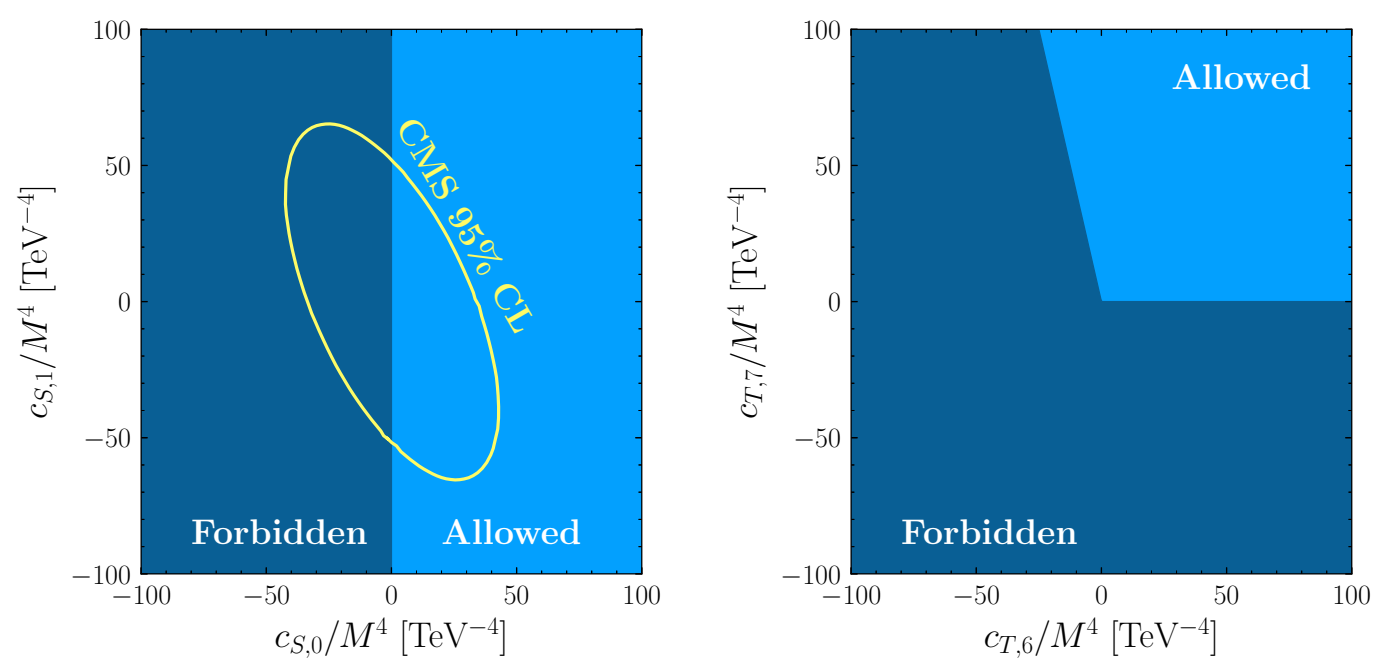

Figure 10. Two examples of the allowed and forbidden aQGC parameter space, as demarcated by our bounds. In each figure we have marginalized over the operators that are not shown. On the left we show the allowed parameter space in the $c_{S, 0}$ and $c_{S, 1}$ plane, applying the bounds in eq. (6.2). Note that $c_{S, 0}+c_{S, 1}+c_{S, 2}>0$ provides no additional information in this plane once we marginalize over $c_{S, 2}$. In this space, we show the $95 \%$ confidence level (CL) limits derived by CMS [32] under the assumption that $c_{S, 2}$ precisely vanishes. On the right, we provide an example of two parameters relevant for aQGCs where our bounds are more restrictive, $c_{T, 6}$ and $c_{T, 7}$, as determined from eq. (6.6). These figures represent an explicit realization of the schematic goal of our work shown in figure 1 .

At this stage, we have ten bounds that constrain 13 of the aQGC operators (plus one additional bound that cannot be translated as the standard aQGC basis is incomplete). This can be contrasted with refs. [65] and [66], where the authors gave bounds on 16 and 18 operators, respectively. One point of difference is that in the present work we have restricted ourselves to the scattering of individual gauge-bosons in the unbroken phase, with colors chosen subject to the condition in eq. (3.13). A possibility for going beyond this is to consider scattering a superpositions of gauge bosons, which is particularly well motivated given that the $\operatorname{SM} \gamma, Z$, and $W^{ \pm}$are themselves superpositions of the unbroken fields. Fully mapping out in detail the space of all positivity bounds on the operators in tables 1 and 2 arising from arbitrary superpositions of $B, W, G$, and $H$ fields is beyond the scope of our present work, but would proceed similarly to the approach we used for scalars in section 4.3. To provide a flavor of the additional constraints that can be derived from such an approach, however, let us consider a simple example of scattering states of the form $\left(B+W^{3}\right) / \sqrt{2}$, which would be the photon in a theory with $\theta_{W}=\pi / 4$. Doing so, we arrive at the following additional bounds on the operators in table 1 :

$$
\begin{aligned}
& c_{1}^{B^{4}}+c_{1}^{W^{4}}+c_{3}^{W^{4}}+c_{1}^{B^{2} W^{2}}+c_{3}^{B^{2} W^{2}}>0 \\
& c_{2}^{B^{4}}+c_{2}^{W^{4}}+c_{4}^{W^{4}}+c_{2}^{B^{2} W^{2}}+c_{4}^{B^{2} W^{2}}>0 \\
& \left(\tilde{c}_{1}^{B^{4}}+\tilde{c}_{1}^{W^{4}}+\tilde{c}_{2}^{W^{4}}+\tilde{c}_{1}^{B^{2} W^{2}}+\tilde{c}_{2}^{B^{2} W^{2}}+\tilde{c}_{3}^{B^{2} W^{2}}\right)^{2} \\
& \quad<4\left(c_{1}^{B^{4}}+c_{1}^{W^{4}}+c_{3}^{W^{4}}+c_{1}^{B^{2} W^{2}}+c_{3}^{B^{2} W^{2}}\right)\left(c_{2}^{B^{4}}+c_{2}^{W^{4}}+c_{4}^{W^{4}}+c_{2}^{B^{2} W^{2}}+c_{4}^{B^{2} W^{2}}\right) .
\end{aligned}
$$


The first of these can be converted completely into the aQGC notation, and requires

$$
4 g_{2}^{4}\left(2 c_{T, 0}+2 c_{T, 1}+c_{T, 2}\right)+2 g_{1}^{2} g_{2}^{2}\left(2 c_{T, 5}+2 c_{T, 6}+c_{T, 7}\right)+g_{1}^{4}\left(2 c_{T, 8}+c_{T, 9}\right)>0 .
$$

This inequality now provides a constraint on the coefficient of $\mathcal{O}_{T, 5}$, although in a more complicated form than the bounds already presented. More generally, additional superpositions will allow the construction of further bounds than those already considered, but we postpone a full exploration to future work.

Before moving on, let us comment on additional collider searches where our bounds are likely to be relevant. The discussion here will be brief, as it would be appropriate to have the complete set of superposition bounds before determining the full scope of applicability. Fundamentally, any such search must be sensitive to the effect of dimension-eight operators, preferably in a channel where the dimension-six effects can be suppressed. Of course, even if both types of operators are present, it is not true that all UV theories predict that the dimension-six operators dominate. For example, in certain theories that invoke strong dynamics at the $\mathrm{TeV}$ scale, it has been shown that dimension-eight operators can sometimes dominate the corresponding LHC observables [137, 138]. Even in situations where the dimension-six operators are expected to dominate, there are still experimental handles to expose the higher-dimension effects. In the case of aQGCs, as already mentioned, there are separate channels where the dimension-six operators can be independently constrained. Another possibility would involve observables where the leading contribution occurs at dimension eight. Such a possibility is provided by neutral triple gauge boson couplings [139]; future $e^{+} e^{-}$colliders will be particularly sensitive to a possible $Z Z \gamma$ vertex [140].

More generically, the contribution from higher-dimension operators is expected to fall less quickly with $s$, and thereby can be isolated by studying the kinematic tails of distributions. A classic example of this, discussed in refs. [141, 142], is that if the Superconducting Super Collider had been realized, the effect of dimension-eight pure gluon operators of the form $G^{4}$ could be observed in the high $p_{T}$ tail of the inclusive differential jet cross section, $p p \rightarrow$ jet $+X$. This effect can also manifest at the LHC. In ref. [87], the authors considered the systematic error induced by neglecting dimension-eight operators when extracting coefficients for the dimension-six corrections to $p p \rightarrow h W^{+}$. They found that, depending on the measured deviation from the SM for $\sigma\left(p p \rightarrow h W^{+}\right)$, the associated error was generally on the order of several percent. Nevertheless, if the analysis were repeated with an invariant mass cut of $m_{h W}>500 \mathrm{GeV}$, the error increased to as large as $50 \%$, highlighting the increased relevance of dimension-eight operators in the tails. This approach can also be pursued to probe SMEFT corrections to the Higgs couplings [134]. Despite these possibilities, there are disadvantages inherent in focusing on the tails, such as the inherent reduction in statistics and further the enhanced PDF uncertainties (for an example see ref. [143]), although this latter uncertainty can potentially be reduced by considering cross section ratios instead. ${ }^{24}$

\subsection{Neutron EDM}

The form in which CP-odd operators enter our results, always being bounded by the associated CP-even contributions, raises the intriguing possibility of connecting potentially

\footnotetext{
${ }^{24}$ We thank Jesse Thaler for this suggestion.
} 
disparate experimental measurements. A comprehensive analysis of this possibility is beyond our present scope, but in this section we demonstrate a proof-of-principle example: the neutron EDM.

The coefficient of the dimension-four operator that would generate a neutron EDM, $G \widetilde{G}$, is famously small, which raises the possibility of a dominant contribution arising from higher-dimension operators. Indeed, as first observed in ref. [144], in extensions to the SM involving multiple Higgs, at low energies the neutron EDM receives a large contribution from the following dimension-six operator from table 3:

$$
\widetilde{\mathcal{O}}_{\operatorname{dim}-6}^{G^{3}}=f^{a b c} G_{\mu}^{a \nu} G_{\nu}^{b \rho} \widetilde{G}_{\rho}^{c \mu} .
$$

The low energy SMEFT associated with these models also includes several of the dimensioneight CP-odd operators considered in this work, namely $\widetilde{\mathcal{O}}_{1-3}^{G^{4}}$. In ref. [145], it was shown that in these same multi-Higgs scenarios, it is possible for the dimension-eight operators to actually dominate over the dimension-six in the contribution to the neutron EDM, highlighting this as an observable potentially sensitive to CP-odd operators that are subject to our bounds. As another example, in ref. [146] it was demonstrated that in a theory containing a heavy quark with a chromoelectric dipole moment, the corresponding EFT at low energies generates both $\widetilde{\mathcal{O}}_{1}^{G^{4}}$ and $\widetilde{\mathcal{O}}_{3}^{G^{4}}$. (It can be verified that in this case the EFT coefficients explicitly satisfy our bounds.)

Recall that the bounds we derived on these operators in eq. (4.14), absent any fine tuning, are schematically of the form $\left(\widetilde{c}^{G^{4}}\right)^{2}<\left(c^{G^{4}}\right)^{2}$; heuristically, the CP-even contribution bounds the CP-odd. Yet as seen above, experiment should also bound the CP-odd contribution in this case. To estimate the impact of the dimension-eight operators on the neutron EDM, following ref. [144], we can use naive dimensional analysis [147, 148] applied to an operator of the form $\left(c / M^{4}\right) G^{3} \widetilde{G}$. Doing so, we obtain

$$
\left|d_{n}\right| \sim \frac{e c}{M^{4}} \frac{\Lambda_{\chi \mathrm{SB}}^{3}}{(4 \pi)^{2}} \approx c\left(\frac{1 \mathrm{TeV}}{M}\right)^{4} \times 10^{-28} e \mathrm{~cm} .
$$

Here $\Lambda_{\chi \mathrm{SB}}=4 \pi F_{\pi} \approx 1190 \mathrm{MeV}$ is the scale associated with chiral symmetry breaking. Taking $c \sim \mathcal{O}(1)$ and the current experimental bound of $\left|d_{n}\right| \lesssim 10^{-26} e \mathrm{~cm}$ [14-16], we see these measurements are already probing the scale associated with $\widetilde{\mathcal{O}}_{1-3}^{G^{4}}$ to several hundred GeV.

The hope would then be to compare this estimate to the limits on the associated CPeven contribution. Unfortunately, bounds on CP-even dimension-eight gluon operators, for example from multijet events at colliders, have not been comprehensively considered in the literature; as such, we cannot explore this possibility here. We note that there are clear challenges inherent in such an analysis. Most importantly there are significant uncertainties associated with measurements of multijet cross sections at the LHC (see, for example, refs. $[149,150]$ ), on top of which a small correction from dimension-eight SMEFT operators would be difficult to constrain. Nevertheless, this example is a proof of principle for how our CP-odd bounds could be utilized. Another possible consequence of our bounds, in this case, is if a definitive neutron EDM were discovered, one way to test if it had a 
dimension-eight origin would be to search for the associated CP-even effect, the magnitude of which can be estimated from the same bound.

\section{Conclusions}

In this paper, we have placed bounds on operator coefficients in the SMEFT. For the 64 quartic bosonic operators with four derivatives at mass dimension eight enumerated in section 3 (see tables 1 and 2), we derived 27 independent bounds on their Wilson coefficients in section 4 , which we restate here for convenience: ${ }^{25}$

$$
\begin{array}{rlrl}
3 c_{1}^{G^{4}}+3 c_{3}^{G^{4}}+c_{5}^{G^{4}} & >0 & & c_{3}^{B^{2} W^{2}}>0 \\
3 c_{3}^{G^{4}}+2 c_{5}^{G^{4}} & >0 & & c_{4}^{B^{2} W^{2}}>0 \\
3 c_{2}^{G^{4}}+3 c_{4}^{G^{4}}+c_{6}^{G^{4}} & >0 & c_{3}^{B^{2} G^{2}}>0 \\
3 c_{4}^{G^{4}}+2 c_{6}^{G^{4}} & >0 & c_{4}^{B^{2} G^{2}}>0 \\
c_{1}^{W^{4}}+c_{3}^{W^{4}} & >0 & & c_{3}^{W^{2} G^{2}}>0 \\
c_{2}^{W^{4}}+c_{4}^{W^{4}} & >0 & & c_{4}^{W^{2} G^{2}}>0 \\
c_{1}^{B^{4}} & >0 & & c_{1}^{H^{2} B^{2}}>0 \\
c_{2}^{B^{4}} & >0 & & c_{1}^{H^{2} W^{2}}>0 \\
c_{1}^{H^{4}}+c_{2}^{H^{4}}+c_{3}^{H^{4}} & >0 & & c_{1}^{H^{2} G^{2}}>0 \\
c_{1}^{H^{4}}+c_{2}^{H^{4}} & >0 & \\
c_{2}^{H^{4}} & >0 &
\end{array}
$$

and

$$
\begin{aligned}
\left(3 \widetilde{c}_{1}^{G^{4}}+3 \widetilde{c}_{2}^{G^{4}}+\widetilde{c}_{3}^{G^{4}}\right)^{2} & <4\left(3 c_{1}^{G^{4}}+3 c_{3}^{G^{4}}+c_{5}^{G^{4}}\right)\left(3 c_{2}^{G^{4}}+3 c_{4}^{G^{4}}+c_{6}^{G^{4}}\right) \\
\left(3 \widetilde{c}_{2}^{G^{4}}+2 \widetilde{c}_{3}^{G^{4}}\right)^{2} & <4\left(3 c_{3}^{G^{4}}+2 c_{5}^{G^{4}}\right)\left(3 c_{4}^{G^{4}}+2 c_{6}^{G^{4}}\right) \\
\left(\widetilde{c}_{1}^{W^{4}}+\widetilde{c}_{2}^{W^{4}}\right)^{2} & <4\left(c_{1}^{W^{4}}+c_{3}^{W^{4}}\right)\left(c_{2}^{W^{4}}+c_{4}^{W^{4}}\right) \\
\left(\widetilde{c}_{1}^{B^{4}}\right)^{2} & <4 c_{1}^{B^{4}} c_{2}^{B^{4}} \\
\left(\widetilde{c}_{3}^{B^{2} W^{2}}\right)^{2} & <4 c_{3}^{B^{2} W^{2}} c_{4}^{B^{2} W^{2}} \\
\left(\widetilde{c}_{3}^{B^{2} G^{2}}\right)^{2} & <4 c_{3}^{B^{2} G^{2}} c_{4}^{B^{2} G^{2}} \\
\left(\widetilde{c}_{3}^{W^{2}} G^{2}\right)^{2} & <4 c_{3}^{W^{2} G^{2}} c_{4}^{W^{2} G^{2}} .
\end{aligned}
$$

As discussed in sections 2 and 4, these bounds follow from amplitudes and causality arguments analogous to those of ref. [34]: analyticity and unitarity of two-to-two scattering amplitudes, along with subluminal signal propagation in the EFT in nontrivial backgrounds. While IR consistency techniques have been previously used to constrain various EFTs of interest [34, 38-60, 65-71], they hitherto have not been systematically applied to the full

\footnotetext{
${ }^{25}$ We reiterate that in this work we have used the mostly-plus metric convention. Working instead in the mostly-minus convention, these results are identical except for three sign changes, resulting in $c_{1}^{H^{2} B^{2}}<0$, $c_{1}^{H^{2} W^{2}}<0$, and $c_{1}^{H^{2} G^{2}}<0$.
} 
SMEFT itself. As a test of our bounds, in section 5 we exhibited a litany of UV completions of these operators and found that our inequalities were always satisfied. The IR requirements of analyticity and causality are relatively agnostic of the details of the UV completion and therefore provide a robust means of bounding the coefficients of deformations of the low-energy EFT. They are hence highly relevant to the current situation in particle physics. Indeed, as discussed in section 6 , the bounds derived in this paper will be useful in any search that can be cast in terms of the operators we constrain. As two explicit examples, we demonstrated the immediate applicability of our results to aQGC searches at the LHC and measurements of the neutron EDM.

The results of this paper suggest many well-motivated directions for future study. While in the present work we have restricted ourselves to scattering amplitudes for boson states of definite type $(H, B, W$, or $G)$, it would be very interesting to apply the optical theorem to incoming states that are superpositions of different bosons (e.g., a superposition of a Higgs and a gluon), which would result in yet more positivity bounds on the EFT coefficients. However, this presents a mathematical challenge, since marginalizing over the space of superposition coefficients to find the minimal set of required bounds amounts to determining positivity of a quartic form, a problem known to be analytically strongly NP-hard [151] (see also ref. [44]). Beyond this, there is significant scope to explore the experimental consequences of our existing (and potentially superposition-extended) bounds. In particular, the possibility of finding additional connections between a CP-even and odd measurement is an especially compelling direction. Another exciting future direction would be to derive bounds on operators that include fermion fields in the SMEFT. Finally, we could consider constraints on operator coefficients arising from analyticity and dispersion relations beyond the forward limit [55] — including the beyond-positivity methods of ref. [47] and the EFThedron construction of ref. [152] — which could provide yet more bounds on Wilson coefficients. We leave these interesting questions of generalization and application of our SMEFT bounds to future work.

\section{Acknowledgments}

We thank Brando Bellazzini, Cliff Cheung, Nathaniel Craig, Markus Luty, Adam Martin, Tom Melia, Francesco Riva, Dean Robinson, David Shih, Yotam Soreq, Dave Sutherland, Jesse Thaler, Cen Zhang, Zhengkang Zhang, and Shuang-Yong Zhou for useful discussions and comments. G.N.R. and N.L.R. are supported by the Miller Institute for Basic Research in Science at the University of California, Berkeley. The work of G.N.R. was performed in part at the Aspen Center for Physics, which is supported by National Science Foundation grant PHY-1607611.

\section{A Group theory definitions}

In section 5.1, we considered completions of the CP-conserving operators in table 1 by integrating out at one loop a massive state $\Phi$ charged under an arbitrary irreducible representation of the SM gauge group. Accordingly, the Wilson coefficients in table 5 depend 
on various group theoretic quantities. In this appendix, we define these quantities in more detail. Throughout, we adopt the conventions of ref. [109]. For an arbitrary irreducible representation $\mathbf{R}$ of gauge group $\mathrm{SU}(N)$ for arbitrary $N$, we write $T_{\mathbf{R}}$ for the generators of $\mathbf{R}$ and define the structure constants as

$$
\left[T_{\mathbf{R}}^{a}, T_{\mathbf{R}}^{b}\right]=i f^{a b c} T_{\mathbf{R}}^{c}
$$

We can define the quadratic, cubic, and quartic invariants $I_{2,3,4}$ via

$$
\begin{aligned}
\operatorname{Tr}\left(T_{\mathbf{R}}^{(a} T_{\mathbf{R}}^{b)}\right) & =I_{2}(\mathbf{R}) \delta^{a b} \\
\operatorname{Tr}\left(T_{\mathbf{R}}^{(a} T_{\mathbf{R}}^{b} T_{\mathbf{R}}^{c)}\right) & =\frac{1}{4} I_{3}(\mathbf{R}) d^{a b c} \\
\operatorname{Tr}\left(T_{\mathbf{R}}^{(a} T_{\mathbf{R}}^{b} T_{\mathbf{R}}^{c} T_{\mathbf{R}}^{d)}\right) & =I_{4}(\mathbf{R}) d^{a b c d}+\Lambda(\mathbf{R})\left(\delta^{a b} \delta^{c d}+\delta^{a c} \delta^{b d}+\delta^{a d} \delta^{b c}\right) .
\end{aligned}
$$

The generators are normalized such that $I_{2}(\mathbf{F})=1 / 2$, where $\mathbf{F}$ denotes the fundamental representation, i.e., the representation of dimension $N$, and the $d^{a b c}$ are normalized such that $I_{3}(\mathbf{F})=1$. The quartic symbol $d^{a b c d}$ in eq. (A.2), which vanishes for $N=2,3$, is given by

$$
d^{a b c d}=\frac{1}{24}\left(d^{a b e} d^{c d e}+d^{a c e} d^{b d e}+d^{a d e} d^{b c e}\right)-\frac{(N+2)(N-2)}{12 N\left(N^{2}+1\right)}\left(\delta^{a b} \delta^{c d}+\delta^{a c} \delta^{b d}+\delta^{a d} \delta^{b c}\right)
$$

and the constant $\Lambda(\mathbf{R})$ in eq. (A.2) is defined as

$$
\Lambda(\mathbf{R})=\left[\frac{D(\mathbf{A}) I_{2}(\mathbf{R})}{D(\mathbf{R})}-\frac{I_{2}(\mathbf{A})}{6}\right] \frac{I_{2}(\mathbf{R})}{2+D(\mathbf{A})},
$$

where $D(\mathbf{R})$ denotes the dimension of the representation and $\mathbf{A}$ denotes the adjoint representation, for which $D(\mathbf{A})=N^{2}-1$ and $I_{2}(\mathbf{A})=N$. Thus, $I_{4}(\mathbf{F})=1$ and $\Lambda(\mathbf{F})=$ $\left(2 N^{2}-3\right) / 12 N\left(N^{2}+1\right)$. The definition in eq. (A.4), along with the third line of eq. (A.2), ensures that $I_{4}(\mathbf{R})=0$ for $N=2,3$.

In order to evaluate these quantities for specific representations, we make use of the fact that an arbitrary irreducible representation $\mathbf{R}$ of $\mathrm{SU}(N)$ can be specified by its Dynkin indices $\left(a_{1}, a_{2}, \ldots, a_{N-1}\right)$, an ordered vector of $N-1$ nonnegative integers. The fundamental, antifundamental, and singlet representations are written as $(1,0, \ldots, 0),(0, \ldots, 0,1)$, and $(0, \ldots, 0)$, respectively. The dimension of the representation is [153]

$$
\begin{aligned}
D(\mathbf{R})= & \prod_{p=1}^{N-1}\left\{\frac{1}{p !} \prod_{q=p}^{N-1}\left[\sum_{r=q-p+1}^{p}\left(1+a_{r}\right)\right]\right\} \\
= & \left(1+a_{1}\right)\left(1+a_{2}\right) \cdots\left(1+a_{N-1}\right) \\
& \times\left(1+\frac{a_{1}+a_{2}}{2}\right)\left(1+\frac{a_{2}+a_{3}}{2}\right) \cdots\left(1+\frac{a_{N-2}+a_{N-1}}{2}\right) \\
& \times\left(1+\frac{a_{1}+a_{2}+a_{3}}{3}\right)\left(1+\frac{a_{2}+a_{3}+a_{4}}{3}\right) \cdots\left(1+\frac{a_{N-3}+a_{N-2}+a_{N-1}}{3}\right) \\
& \vdots \\
& \times\left(1+\frac{a_{1}+\cdots+a_{N-1}}{N-1}\right) .
\end{aligned}
$$


The quadratic invariant is

$$
I_{2}(\mathbf{R})=\frac{D(\mathbf{R}) C_{2}(\mathbf{R})}{2(N+1) N(N-1)},
$$

where $C_{2}(\mathbf{R})$ is the quadratic Casimir, defined via $2 N T_{\mathbf{R}}^{a} T_{\mathbf{R}}^{a}=C_{2}(\mathbf{R}) \mathbb{1}$, or explicitly [153],

$$
C_{2}(\mathbf{R})=\sum_{m=1}^{N-1}\left[N(N-m) m a_{m}+m(N-m) a_{m}^{2}+\sum_{n=0}^{n-1} 2 n(N-m) a_{n} a_{m}\right] .
$$

One can show that $I_{2}(\mathbf{R}) \geq D(\mathbf{R}) / 2 N$. The cubic anomaly invariant is

$$
I_{3}(\mathbf{R})=D(\mathbf{R}) \sum_{p=1}^{N-1} \sum_{q=1}^{N-1} \sum_{r=1}^{N-1} b_{p q r}\left(1+a_{p}\right)\left(1+a_{q}\right)\left(1+a_{r}\right)
$$

where for $i \leq j \leq k$,

$$
b_{p q r}=\frac{2(N-3) !}{(N+2) !} p(N-2 q)(N-r),
$$

and all the other entries are determined by requiring that $b_{p q r}$ be totally symmetric [111]. Finally, the quartic coefficient $I_{4}(\mathbf{R})$ for general $N$ can be found in ref. [154]; since it vanishes for the SM gauge group, it does not impact the operators we consider.

Open Access. This article is distributed under the terms of the Creative Commons Attribution License (CC-BY 4.0), which permits any use, distribution and reproduction in any medium, provided the original author(s) and source are credited.

\section{References}

[1] ATLAS collaboration, Observation of a new particle in the search for the standard model Higgs boson with the ATLAS detector at the LHC, Phys. Lett. B 716 (2012) 1 [arXiv: 1207.7214] [INSPIRE].

[2] CMS collaboration, Observation of a new boson at a Mass of $125 \mathrm{GeV}$ with the CMS experiment at the LHC, Phys. Lett. B $\mathbf{7 1 6}$ (2012) 30 [arXiv:1207.7235] [INSPIRE].

[3] O. Buchmueller and P. de Jong, Supersymmetry, Part II (experiment), in Review of particle physics, Particle Data Group collabroation, Phys. Rev. D 98 (2018) 030001 [INSPIRE].

[4] G.F. Giudice, The dawn of the post-naturalness era, in From my vast repertoire...: Guido Altarelli's legacy, A. Levy, S. Forte and G. Ridolfi eds., World Scientific, Singapore (2019), arXiv: 1710.07663, [INSPIRE].

[5] J.L. Feng, Naturalness and the status of supersymmetry, Ann. Rev. Nucl. Part. Sci. 63 (2013) 351 [arXiv: 1302.6587] [inSPIRE].

[6] C. Cheung and G.N. Remmen, Naturalness and the weak gravity conjecture, Phys. Rev. Lett. 113 (2014) 051601 [arXiv: 1402.2287] [INSPIRE].

[7] S. Dimopoulos, LHC, SSC and the universe, Phys. Lett. B 246 (1990) 347 [INSPIRE].

[8] R. Percacci, Asymptotic safety, arXiv:0709.3851 [INSPIRE]. 
[9] ATLAS, CMS collaboration, Report on the physics at the HL-LHC and perspectives for the HE-LHC, arXiv: 1902.10229 [INSPIRE].

[10] Working Group 3 collaboration, Beyond the standard model physics at the HL-LHC and $H E-L H C$, arXiv: 1812.07831 [INSPIRE].

[11] J. Gao, L. Harland-Lang and J. Rojo, The structure of the proton in the LHC precision era, Phys. Rept. 742 (2018) 1 [arXiv:1709.04922] [inSPIRE].

[12] J. Erler and M. Schott, Electroweak precision tests of the standard model after the discovery of the Higgs boson, Prog. Part. Nucl. Phys. 106 (2019) 68 [arXiv:1902.05142] [inSPIRE].

[13] M. Reece, Physics at a Higgs factory, Int. J. Mod. Phys. A 31 (2016) 1644003 [arXiv: 1609.03018] [INSPIRE].

[14] C.A. Baker et al., An improved experimental limit on the electric dipole moment of the neutron, Phys. Rev. Lett. 97 (2006) 131801 [hep-ex/0602020] [INSPIRE].

[15] J.M. Pendlebury et al., Revised experimental upper limit on the electric dipole moment of the neutron, Phys. Rev. D 92 (2015) 092003 [arXiv:1509.04411] [InSPIRE].

[16] B. Graner, Y. Chen, E.G. Lindahl and B.R. Heckel, Reduced limit on the permanent electric dipole moment of ${ }^{199} \mathrm{Hg}$, Phys. Rev. Lett. 116 (2016) 161601 [Erratum ibid. 119 (2017) 119901] [arXiv: 1601.04339] [INSPIRE].

[17] ACME collaboration, Improved limit on the electric dipole moment of the electron, Nature 562 (2018) 355 [INSPIRE].

[18] ACME collaboration, Order of magnitude smaller limit on the electric dipole moment of the electron, Science 343 (2014) 269 [arXiv: 1310.7534] [INSPIRE].

[19] W.B. Cairncross et al., Precision measurement of the electron's electric dipole moment using trapped molecular ions, Phys. Rev. Lett. 119 (2017) 153001 [arXiv:1704.07928] [INSPIRE].

[20] MuOn G-2 collaboration, Final report of the muon E821 anomalous magnetic moment measurement at BNL, Phys. Rev. D 73 (2006) 072003 [hep-ex/0602035] [INSPIRE].

[21] Muon G-2 collaboration, Muon $(g-2)$ technical design report, arXiv:1501.06858 [INSPIRE].

[22] B. Grzadkowski, M. Iskrzynski, M. Misiak and J. Rosiek, Dimension-six terms in the standard model lagrangian, JHEP 10 (2010) 085 [arXiv: 1008.4884] [INSPIRE].

[23] B. Henning, X. Lu, T. Melia and H. Murayama, 2, 84, 30, 993, 560, 15456, 11962, 261485, ...: higher dimension operators in the SM EFT, JHEP 08 (2017) 016 [Erratum ibid. 09 (2019) 019] [arXiv: 1512.03433] [INSPIRE].

[24] R.M. Fonseca, Enumerating the operators of an effective field theory, arXiv:1907.12584 [INSPIRE].

[25] I. Brivio and M. Trott, The standard model as an effective field theory, Phys. Rept. 793 (2019) 1 [arXiv: 1706 .08945] [INSPIRE].

[26] R. Contino et al., On the validity of the effective field theory approach to SM precision tests, JHEP 07 (2016) 144 [arXiv: 1604.06444] [INSPIRE].

[27] ATLAS collaboration, Evidence for light-by-light scattering in heavy-ion collisions with the ATLAS detector at the LHC, Nature Phys. 13 (2017) 852 [arXiv:1702.01625] [INSPIRE]. 
[28] ATLAS collaboration, Observation of electroweak $W^{ \pm} Z$ boson pair production in association with two jets in pp collisions at $\sqrt{s}=13 \mathrm{TeV}$ with the ATLAS detector, Phys. Lett. B 793 (2019) 469 [arXiv: 1812.09740] [INSPIRE].

[29] ATLAS collaboration, Observation of electroweak production of a same-sign $W$ boson pair in association with two jets in pp collisions at $\sqrt{s}=13 \mathrm{TeV}$ with the ATLAS detector, Phys. Rev. Lett. 123 (2019) 161801 [arXiv:1906.03203] [INSPIRE].

[30] CMS collaboration, Measurement of vector boson scattering and constraints on anomalous quartic couplings from events with four leptons and two jets in proton-roton collisions at $\sqrt{s}=13 \mathrm{TeV}$, Phys. Lett. B 774 (2017) 682 [arXiv:1708.02812] [inSPIRE].

[31] CMS collaboration, Observation of electroweak production of same-sign $W$ boson pairs in the two jet and two same-sign lepton final state in proton-proton collisions at $\sqrt{s}=13 \mathrm{TeV}$, Phys. Rev. Lett. 120 (2018) 081801 [arXiv:1709.05822] [INSPIRE].

[32] CMS collaboration, Measurement of electroweak $W Z$ boson production and search for new physics in $W Z+$ two jets events in pp collisions at $\sqrt{s}=13 \mathrm{TeV}$, Phys. Lett. B 795 (2019) 281 [arXiv: 1901.04060] [INSPIRE].

[33] J. Ellis, C.W. Murphy, V. Sanz and T. You, Updated global SMEFT fit to Higgs, diboson and electroweak data, JHEP 06 (2018) 146 [arXiv:1803.03252] [INSPIRE].

[34] A. Adams et al., Causality, analyticity and an IR obstruction to UV completion, JHEP 10 (2006) 014 [hep-th/0602178] [INSPIRE].

[35] T.N. Pham and T.N. Truong, Evaluation of the derivative quartic terms of the meson chiral lagrangian from forward dispersion relation, Phys. Rev. D 31 (1985) 3027 [INSPIRE].

[36] B. Ananthanarayan, D. Toublan and G. Wanders, Consistency of the chiral pion pion scattering amplitudes with axiomatic constraints, Phys. Rev. D 51 (1995) 1093 [hep-ph/9410302] [INSPIRE].

[37] M.R. Pennington and J. Portoles, The chiral lagrangian parameters, $\ell_{1}, \ell_{2}$, are determined by the $\rho$-resonance, Phys. Lett. B 344 (1995) 399 [hep-ph/9409426] [INSPIRE].

[38] A. Jenkins and D. O'Connell, The story of $\mathcal{O}$ : positivity constraints in effective field theories, hep-th/0609159 [INSPIRE].

[39] G. Dvali, A. Franca and C. Gomez, Road signs for UV-completion, arXiv:1204.6388 [INSPIRE].

[40] B. Bellazzini, C. Cheung and G.N. Remmen, Quantum gravity constraints from unitarity and analyticity, Phys. Rev. D 93 (2016) 064076 [arXiv:1509.00851] [INSPIRE].

[41] C. Cheung and G.N. Remmen, Positivity of curvature-squared corrections in gravity, Phys. Rev. Lett. 118 (2017) 051601 [arXiv: 1608.02942] [INSPIRE].

[42] X.O. Camanho, J.D. Edelstein, J. Maldacena and A. Zhiboedov, Causality constraints on corrections to the graviton three-point coupling, JHEP 02 (2016) 020 [arXiv:1407.5597] [INSPIRE].

[43] A. Gruzinov and M. Kleban, A note on causality constrains higher curvature corrections to gravity, Class. Quant. Grav. 24 (2007) 3521 [hep-th/0612015] [INSPIRE].

[44] C. Cheung and G.N. Remmen, Positive signs in massive gravity, JHEP 04 (2016) 002 [arXiv: 1601.04068] [INSPIRE]. 
[45] C. de Rham, S. Melville and A.J. Tolley, Improved positivity bounds and massive gravity, JHEP 04 (2018) 083 [arXiv: 1710.09611] [INSPIRE].

[46] X.O. Camanho, G. Lucena Gómez and R. Rahman, Causality constraints on massive gravity, Phys. Rev. D 96 (2017) 084007 [arXiv: 1610.02033] [InSPIRE].

[47] B. Bellazzini, F. Riva, J. Serra and F. Sgarlata, Beyond positivity bounds and the fate of massive gravity, Phys. Rev. Lett. 120 (2018) 161101 [arXiv:1710.02539] [INSPIRE].

[48] B. Bellazzini, Softness and amplitudes' positivity for spinning particles, JHEP 02 (2017) 034 [arXiv: 1605.06111] [INSPIRE].

[49] J. Bonifacio and K. Hinterbichler, Bounds on amplitudes in effective theories with massive spinning particles, Phys. Rev. D 98 (2018) 045003 [arXiv: 1804.08686] [INSPIRE].

[50] J. Bonifacio, K. Hinterbichler and R.A. Rosen, Positivity constraints for pseudolinear massive spin-2 and vector Galileons, Phys. Rev. D 94 (2016) 104001 [arXiv:1607.06084] [INSPIRE].

[51] C. de Rham, S. Melville, A.J. Tolley and S.-Y. Zhou, UV complete me: positivity bounds for particles with spin, JHEP 03 (2018) 011 [arXiv: 1706.02712] [INSPIRE].

[52] K. Hinterbichler, A. Joyce and R.A. Rosen, Massive spin-2 scattering and asymptotic superluminality, JHEP 03 (2018) 051 [arXiv: 1708.05716] [INSPIRE].

[53] C. de Rham, S. Melville, A.J. Tolley and S.-Y. Zhou, Positivity bounds for massive spin-1 and spin-2 fields, JHEP 03 (2019) 182 [arXiv:1804.10624] [INSPIRE].

[54] B. Bellazzini, F. Riva, J. Serra and F. Sgarlata, Massive higher spins: effective theory and consistency, JHEP 10 (2019) 189 [arXiv:1903.08664] [INSPIRE].

[55] A. Nicolis, R. Rattazzi and E. Trincherini, Energy's and amplitudes' positivity, JHEP 05 (2010) 095 [Erratum ibid. 11 (2011) 128] [arXiv:0912.4258] [INSPIRE].

[56] H. Elvang et al., On renormalization group flows and the a-theorem in 6d, JHEP 10 (2012) 011 [arXiv: 1205.3994] [INSPIRE].

[57] C. de Rham, S. Melville, A.J. Tolley and S.-Y. Zhou, Massive galileon positivity bounds, JHEP 09 (2017) 072 [arXiv: 1702.08577] [INSPIRE].

[58] V. Chandrasekaran, G.N. Remmen and A. Shahbazi-Moghaddam, Higher-point positivity, JHEP 11 (2018) 015 [arXiv: 1804.03153] [INSPIRE].

[59] M. Herrero-Valea, I. Timiryasov and A. Tokareva, To positivity and beyond, where Higgs-dilaton inflation has never gone before, arXiv:1905.08816 [INSPIRE].

[60] Z. Komargodski and A. Schwimmer, On renormalization group flows in four dimensions, JHEP 12 (2011) 099 [arXiv:1107.3987] [InSPIRE].

[61] B. Bellazzini, F. Riva, J. Serra and F. Sgarlata, The other effective fermion compositeness, JHEP 11 (2017) 020 [arXiv:1706.03070] [INSPIRE].

[62] J. Distler, B. Grinstein, R.A. Porto and I.Z. Rothstein, Falsifying models of new physics via WW scattering, Phys. Rev. Lett. 98 (2007) 041601 [hep-ph/0604255] [INSPIRE].

[63] L. Vecchi, Causal versus analytic constraints on anomalous quartic gauge couplings, JHEP 11 (2007) 054 [arXiv: 0704.1900] [INSPIRE].

[64] B. Bellazzini and F. Riva, New phenomenological and theoretical perspective on anomalous $Z Z$ and $Z \gamma$ processes, Phys. Rev. D 98 (2018) 095021 [arXiv: 1806.09640] [InSPIRE]. 
[65] C. Zhang and S.-Y. Zhou, Positivity bounds on vector boson scattering at the LHC, Phys. Rev. D 100 (2019) 095003 [arXiv: 1808.00010] [INSPIRE].

[66] Q. Bi, C. Zhang and S.-Y. Zhou, Positivity constraints on aQGC: carving out the physical parameter space, JHEP 06 (2019) 137 [arXiv:1902.08977] [INSPIRE].

[67] C. Cheung and G.N. Remmen, Infrared consistency and the weak gravity conjecture, JHEP 12 (2014) 087 [arXiv: 1407.7865] [INSPIRE].

[68] C. Cheung, J. Liu and G.N. Remmen, Proof of the weak gravity conjecture from black hole entropy, JHEP 10 (2018) 004 [arXiv:1801.08546] [INSPIRE].

[69] C. Cheung, J. Liu and G.N. Remmen, Entropy bounds on effective field theory from rotating dyonic black holes, Phys. Rev. D 100 (2019) 046003 [arXiv:1903.09156] [InSPIRE].

[70] B. Bellazzini, M. Lewandowski and J. Serra, Amplitudes' positivity, weak gravity conjecture and modified gravity, arXiv: 1902.03250 [INSPIRE].

[71] A.M. Charles, The weak gravity conjecture, $R G$ flows and supersymmetry, arXiv:1906.07734 [INSPIRE].

[72] C. Vafa, The string landscape and the swampland, hep-th/0509212 [INSPIRE].

[73] H. Ooguri and C. Vafa, On the geometry of the string landscape and the swampland, Nucl. Phys. B 766 (2007) 21 [hep-th/0605264] [INSPIRE].

[74] N. Arkani-Hamed, L. Motl, A. Nicolis and C. Vafa, The string landscape, black holes and gravity as the weakest force, JHEP 06 (2007) 060 [hep-th/0601001] [INSPIRE].

[75] D.R. Green, P. Meade and M.-A. Pleier, Multiboson interactions at the LHC, Rev. Mod. Phys. 89 (2017) 035008 [arXiv:1610.07572] [INSPIRE].

[76] O.J.P. Eboli, M.C. Gonzalez-Garcia and J.K. Mizukoshi, $p p \rightarrow j j e^{ \pm} \mu^{ \pm} \nu \nu$ and $j j e^{ \pm} \mu^{\mp} \nu \nu$ at $\mathcal{O}\left(\alpha_{\mathrm{em}}^{6}\right)$ and $\mathcal{O}\left(\alpha_{\mathrm{em}}^{4} \alpha_{s}^{2}\right)$ for the study of the quartic electroweak gauge boson vertex at CERN LHC, Phys. Rev. D 74 (2006) 073005 [hep-ph/0606118] [InSPIRE].

[77] I. Low, R. Rattazzi and A. Vichi, Theoretical constraints on the Higgs effective couplings, JHEP 04 (2010) 126 [arXiv:0907.5413] [InSPIRE].

[78] C. Englert, G.F. Giudice, A. Greljo and M. Mccullough, The $\hat{H}$-parameter: an oblique Higgs view, JHEP 09 (2019) 041 [arXiv:1903.07725] [INSPIRE].

[79] M. Froissart, Asymptotic behavior and subtractions in the Mandelstam representation, Phys. Rev. 123 (1961) 1053 [INSPIRE].

[80] A. Martin, Unitarity and high-energy behavior of scattering amplitudes, Phys. Rev. 129 (1963) 1432 [INSPIRE].

[81] A. Martin, Extension of the axiomatic analyticity domain of scattering amplitudes by unitarity. 1., Nuovo Cim. A 42 (1965) 930 [InSPIRE].

[82] S. Mandelstam, Determination of the pion-nucleon scattering amplitude from dispersion relations and unitarity. General theory, Phys. Rev. 112 (1958) 1344 [INSPIRE].

[83] H. Lehmann, Analytic properties of scattering amplitudes as functions of momentum transfer, Nuovo Cim. 10 (1958) 579.

[84] G.A. Benford, D.L. Book and W.A. Newcomb, The tachyonic antitelephone, Phys. Rev. D 2 (1970) 263 [INSPIRE]. 
[85] R.C. Tolman, The theory of relativity of motion, University of California Press, Berkeley, U.S.A. (1917).

[86] A.Yu. Morozov, Matrix of mixing of scalar and vector mesons of dimension $D \leq 8$ in $Q C D$ (in Russian), Sov. J. Nucl. Phys. 40 (1984) 505 [InSPIRE].

[87] C. Hays, A. Martin, V. Sanz and J. Setford, On the impact of dimension-eight SMEFT operators on Higgs measurements, JHEP 02 (2019) 123 [arXiv:1808.00442] [INSPIRE].

[88] A. Martin, private communication (2019).

[89] S. Weinberg, Baryon and lepton nonconserving processes, Phys. Rev. Lett. 43 (1979) 1566 [INSPIRE].

[90] W. Buchmüller and D. Wyler, Effective lagrangian analysis of new interactions and flavor conservation, Nucl. Phys. B 268 (1986) 621 [INSPIRE].

[91] E.E. Jenkins, A.V. Manohar and M. Trott, Renormalization group evolution of the standard model dimension six operators I: formalism and $\lambda$ dependence, JHEP 10 (2013) 087 [arXiv: 1308.2627] [INSPIRE].

[92] E.E. Jenkins, A.V. Manohar and M. Trott, Renormalization group evolution of the standard model dimension six operators II: Yukawa dependence, JHEP 01 (2014) 035 [arXiv: 1310.4838] [INSPIRE].

[93] R. Alonso, E.E. Jenkins, A.V. Manohar and M. Trott, Renormalization group evolution of the standard model dimension six operators III: gauge coupling dependence and phenomenology, JHEP 04 (2014) 159 [arXiv: 1312.2014] [INSPIRE].

[94] E.E. Jenkins, A.V. Manohar and P. Stoffer, Low-energy effective field theory below the electroweak scale: operators and matching, JHEP 03 (2018) 016 [arXiv:1709.04486] [INSPIRE].

[95] E.E. Jenkins, A.V. Manohar and P. Stoffer, Low-energy effective field theory below the electroweak scale: anomalous dimensions, JHEP 01 (2018) 084 [arXiv:1711.05270] [INSPIRE].

[96] H. Elvang and Y.-t. Huang, Scattering amplitudes, arXiv:1308.1697 [INSPIRE].

[97] R. Britto, F. Cachazo and B. Feng, New recursion relations for tree amplitudes of gluons, Nucl. Phys. B 715 (2005) 499 [hep-th/0412308] [INSPIRE].

[98] R. Britto, F. Cachazo, B. Feng and E. Witten, Direct proof of tree-level recursion relation in Yang-Mills theory, Phys. Rev. Lett. 94 (2005) 181602 [hep-th/0501052] [INSPIRE].

[99] N. Arkani-Hamed et al., Grassmannian geometry of scattering amplitudes, Cambridge University Press, Cambridge U.K. (2016) [arXiv: 1212.5605] [INSPIRE].

[100] C. Cheung, G.N. Remmen, C.-H. Shen and C. Wen, Pions as gluons in higher dimensions, JHEP 04 (2018) 129 [arXiv:1709.04932] [INSPIRE].

[101] H. Kawai, D.C. Lewellen and S.H.H. Tye, A relation between tree amplitudes of closed and open strings, Nucl. Phys. B 269 (1986) 1 [inSPIRE].

[102] A. Rebhan and G. Turk, Polarization effects in light-by-light scattering: Euler-Heisenberg versus Born-Infeld, Int. J. Mod. Phys. A 32 (2017) 1750053 [arXiv:1701.07375] [InSPIRE].

[103] G.W. Gibbons and C.A.R. Herdeiro, Born-Infeld theory and stringy causality, Phys. Rev. D 63 (2001) 064006 [hep-th/0008052] [INSPIRE]. 
[104] M. Fouché, R. Battesti and C. Rizzo, Limits on nonlinear electrodynamics, Phys. Rev. D 93 (2016) 093020 [Erratum ibid. D 95 (2017) 099902] [arXiv: 1605.04102] [INSPIRE].

[105] F. Abalos, F. Carrasco, É. Goulart and O. Reula, Nonlinear electrodynamics as a symmetric hyperbolic system, Phys. Rev. D 92 (2015) 084024 [arXiv:1507.02262] [INSPIRE].

[106] W. Heisenberg and H. Euler, Consequences of Dirac's theory of positrons, Z. Phys. 98 (1936) 714 [physics/0605038].

[107] J.S. Schwinger, On gauge invariance and vacuum polarization, Phys. Rev. 82 (1951) 664 [INSPIRE].

[108] V. Weisskopf, The electrodynamics of the vacuum based on the quantum theory of the electron, Kong. Dans. Vid. Selsk. Mat-Fys. Medd. XIV (1936) 1.

[109] J. Quevillon, C. Smith and S. Touati, Effective action for gauge bosons, Phys. Rev. D 99 (2019) 013003 [arXiv: 1810.06994] [INSPIRE].

[110] C.G. Wohl, SU(n) multiplets and Young diagrams, in Review of particle physics, Particle Data Group collabroation, Phys. Rev. D 98 (2018) 030001 [INSPIRE].

[111] J. Banks and H. Georgi, Comment on gauge theories without anomalies, Phys. Rev. D 14 (1976) 1159 [INSPIRE].

[112] M. Born and L. Infeld, Foundations of the new field theory, Proc. Roy. Soc. Lond. A 144 (1934) 425.

[113] E.S. Fradkin and A.A. Tseytlin, Nonlinear electrodynamics from quantized strings, Phys. Lett. B 163 (1985) 123.

[114] A.A. Tseytlin, Born-Infeld action, supersymmetry and string theory, hep-th/9908105 [INSPIRE].

[115] C. Cheung, C.-H. Shen and C. Wen, Unifying relations for scattering amplitudes, JHEP 02 (2018) 095 [arXiv: 1705. 03025] [InSPIRE].

[116] C. Cheung et al., Vector effective field theories from soft limits, Phys. Rev. Lett. 120 (2018) 261602 [arXiv: 1801.01496] [INSPIRE].

[117] J. Ellis and S.-F. Ge, Constraining gluonic quartic gauge coupling operators with $g g \rightarrow \gamma \gamma$, Phys. Rev. Lett. 121 (2018) 041801 [arXiv:1802.02416] [INSPIRE].

[118] J.H. Schwarz, Dilaton-axion symmetry, in the proceedings of the International Workshop on String Theory, Quantum Gravity and the Unification of Fundamental Interactions, September 21-26, Rome, Italy (1992), hep-th/9209125 [INSPIRE].

[119] R. Kallosh, Supergravity, M-theory and cosmology, in the proceedings of The future of theoretical physics and cosmology: Celebrating Stephen Hawking's $60^{\text {th }}$ birthday, January 7-10, Cambridge, U.K. (2002), hep-th/0205315 [INSPIRE].

[120] J.P. Conlon, The QCD axion and moduli stabilisation, JHEP 05 (2006) 078 [hep-th/0602233] [INSPIRE].

[121] X. Gao and P. Shukla, Dimensional oxidation and modular completion of non-geometric type IIB action, JHEP 05 (2015) 018 [arXiv: 1501.07248] [INSPIRE].

[122] S. Endlich, V. Gorbenko, J. Huang and L. Senatore, An effective formalism for testing extensions to General Relativity with gravitational waves, JHEP 09 (2017) 122 [arXiv: 1704.01590] [INSPIRE]. 
[123] K. Hinterbichler, Theoretical aspects of massive gravity, Rev. Mod. Phys. 84 (2012) 671 [arXiv:1105.3735] [INSPIRE].

[124] N.P. Hartland et al., A Monte Carlo global analysis of the Standard Model Effective Field Theory: the top quark sector, JHEP 04 (2019) 100 [arXiv:1901.05965] [INSPIRE].

[125] S. van Beek, E.R. Nocera, J. Rojo and E. Slade, Constraining the SMEFT with Bayesian reweighting, arXiv:1906.05296 [INSPIRE].

[126] V. De Luca et al., Colored dark matter, Phys. Rev. D 97 (2018) 115024 [arXiv: 1801.01135] [INSPIRE].

[127] O.J.P. Eboli, M.C. Gonzalez-Garcia, S.M. Lietti and S.F. Novaes, Anomalous quartic gauge boson couplings at hadron colliders, Phys. Rev. D 63 (2001) 075008 [hep-ph/0009262] [INSPIRE].

[128] D0 collaboration, Search for anomalous quartic $W W \gamma \gamma$ couplings in dielectron and missing energy final states in pp collisions at $\sqrt{s}=1.96 \mathrm{TeV}$, Phys. Rev. D 88 (2013) 012005 [arXiv: 1305.1258] [INSPIRE].

[129] CMS collaboration, Study of exclusive two-photon production of $W^{+} W^{-}$in pp collisions at $\sqrt{s}=7 \mathrm{TeV}$ and constraints on anomalous quartic gauge couplings, JHEP 07 (2013) 116 [arXiv:1305.5596] [INSPIRE].

[130] CMS collaboration, Evidence for exclusive $\gamma \gamma \rightarrow W^{+} W^{-}$production and constraints on anomalous quartic gauge couplings in pp collisions at $\sqrt{s}=7$ and 8 TeV, JHEP 08 (2016) 119 [arXiv: 1604.04464] [INSPIRE].

[131] ATLAS collaboration, Measurements of $Z \gamma$ and $Z \gamma \gamma$ production in pp collisions at $\sqrt{s}=8$ TeV with the ATLAS detector, Phys. Rev. D 93 (2016) 112002 [arXiv:1604.05232] [INSPIRE].

[132] J. Ellis, N.E. Mavromatos and T. You, Light-by-light scattering constraint on Born-Infeld theory, Phys. Rev. Lett. 118 (2017) 261802 [arXiv:1703.08450] [INSPIRE].

[133] A. Butter et al., The gauge-Higgs legacy of the LHC Run I, JHEP 07 (2016) 152 [arXiv: 1604.03105] [INSPIRE].

[134] B. Henning, D. Lombardo, M. Riembau and F. Riva, Higgs couplings without the Higgs, Phys. Rev. Lett. 123 (2019) 181801 [arXiv:1812.09299] [INSPIRE].

[135] C. Degrande et al., Monte Carlo tools for studies of non-standard electroweak gauge boson interactions in multi-boson processes: A Snowmass White Paper, in the proceedings of the 2013 Community Summer Study on the Future of U.S. Particle Physics: Snowmass on the Mississippi (CSS2013), July 29-August 6, Minneapolis, U.S.A. (2013), arXiv:1309.7890 [INSPIRE].

[136] M. Rauch, Vector-boson fusion and vector-boson scattering, arXiv:1610.08420 [INSPIRE].

[137] D. Liu, A. Pomarol, R. Rattazzi and F. Riva, Patterns of strong coupling for LHC searches, JHEP 11 (2016) 141 [arXiv:1603.03064] [INSPIRE].

[138] D. Liu and L.-T. Wang, Prospects for precision measurement of diboson processes in the semileptonic decay channel in future LHC runs, Phys. Rev. D 99 (2019) 055001 [arXiv: 1804.08688] [INSPIRE].

[139] C. Degrande, A basis of dimension-eight operators for anomalous neutral triple gauge boson interactions, JHEP 02 (2014) 101 [arXiv: 1308.6323] [INSPIRE]. 
[140] J. Ellis, S.-F. Ge, H.-J. He and R.-Q. Xiao, Probing the scale of new physics in the $Z Z \gamma$ coupling at $e^{+} e^{-}$colliders, arXiv:1902.06631 [INSPIRE].

[141] E.H. Simmons, Dimension-six gluon operators as probes of new physics, Phys. Lett. B 226 (1989) 132 [inSPIRE].

[142] E.H. Simmons, Higher dimension gluon operators and hadronic scattering, Phys. Lett. B 246 (1990) 471 [INSPIRE].

[143] M. Czakon et al., Top-pair production at the LHC through NNLO QCD and NLO EW, JHEP 10 (2017) 186 [arXiv: 1705. 04105] [INSPIRE].

[144] S. Weinberg, Larger Higgs exchange terms in the neutron electric dipole moment, Phys. Rev. Lett. 63 (1989) 2333 [INSPIRE].

[145] M. Chemtob, Nucleon electric dipole moment and dimension 8 gluonic operators, Phys. Rev. D 48 (1993) 283 [INSPIRE].

[146] D. Chang, T.W. Kephart, W.-Y. Keung and T.C. Yuan, The chromoelectric dipole moment of the heavy quark and purely gluonic CP-violating operators, Phys. Rev. Lett. 68 (1992) 439 [INSPIRE].

[147] A. Manohar and H. Georgi, Chiral quarks and the nonrelativistic quark model, Nucl. Phys. B 234 (1984) 189 [INSPIRE].

[148] H. Georgi and L. Randall, Flavor conserving CP-violation in invisible axion models, Nucl. Phys. B 276 (1986) 241 [INSPIRE].

[149] ATLAS collaboration, Measurement of inclusive jet and dijet cross-sections in proton-proton collisions at $\sqrt{s}=13 \mathrm{TeV}$ with the ATLAS detector, JHEP 05 (2018) 195 [arXiv: 1711.02692] [INSPIRE].

[150] CMS collaboration, Measurement of the triple-differential dijet cross section in proton-proton collisions at $\sqrt{s}=8 \mathrm{TeV}$ and constraints on parton distribution functions, Eur. Phys. J. C 77 (2017) 746 [arXiv: 1705.02628] [INSPIRE].

[151] A. A. Ahmadi, A. Olshevsky, P.A. Parrilo and J.N. Tsitsiklis, NP-hardness of deciding convexity of quartic polynomials and related problems, Math. Prog. 137 (2013) 453 [arXiv: 1012.1908].

[152] N. Arkani-Hamed, T.C. Huang, Y.T. Huang and S.H. Shao, to appear.

[153] D.D. Dietrich and F. Sannino, Conformal window of $\mathrm{SU}(N)$ gauge theories with fermions in higher dimensional representations, Phys. Rev. D 75 (2007) 085018 [hep-ph/0611341] [INSPIRE].

[154] S. Okubo, Modified fourth order casimir invariants and indices for simple Lie algebras, J. Math. Phys. 23 (1982) 8 [INSPIRE]. 